DOE/ID-10057(94)

Distribution Category:

\title{
Idaho National Engineering Laboratory Nonradiological Waste Management Information For 1994 and Record to Date
}

\author{
D. L. French \\ D. J. Liseé \\ K. A. Taylor
}

Published August 1995

DISCLAIMER

This report was prepared as an account of work sponsored by an agency of the United States Government. Neither the United States Government nor any agency thereof, nor any of their employees, makes any warranty, express or implied, or assumes any legal liability or responsibility for the accuracy, completeness, or usefulness of any information, apparatus, product, or process disclosed, or represents that its use would not infringe privately owned rights. Reference herein to any specific commercial product, process, or service by trade name, trademark, manufacturer, or otherwise does not necessarily constitute or imply its endorsement, recommendation, or favoring by the United States Government or any agency thereof. The views and opinions of authors expressed herein do not necessarily state or reflect those of the United States Government or any agency thereof.

\section{Idaho National Engineering Laboratory Lockheed Idaho Technologies Companies \\ Idaho Falls, Idaho 83415}

Prepared for the

U.S. Department of Energy

DOE Idaho Operations Office

Contract No. DE-AC07-94ID13223 


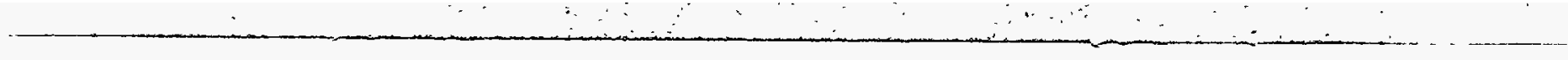

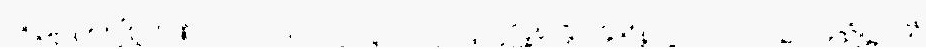




\section{DISCLAIMER}

Portions of this document may be illegible in electronic image products. Images are produced from the best available original document. 


\begin{abstract}
This document provides detailed data and graphics on airborne and liquid effluent releases, fuel oil and coal consumption, water usage, and hazardous and mixed waste generated for calendar year 1994. This report summarizes industrial waste data records compiled since 1971 for the Idaho National Engineering Laboratory (INEL). The data presented are from the INEL Nonradiological Waste Management Information System.
\end{abstract}




\section{EXECUTIVE SUMMARY}

The Idaho National Engineering Laboratory (INEL) Nonradiological Waste Management Information System (INWMIS) is a computerized data base that replaced the Industrial Waste Management Information System (IWMIS) on October 1, 1991. The INWMIS serves as the official record for all types of industrial (nonradiological) waste and is the contact for reporting hazardous and mixed waste stored, disposed, or shipped from the INEL.

U.S. Department of Energy (DOE) contractors routinely report data to the INWMIS. The system provides routine and special reports for nonradiological airborne and liquid effluents, fuel oil consumption, water usage, and storage or disposal of solid wastes.

To more accurately report the landfill data, modifications were made to all landfill reports to include final volumes and gross weight. Currently the landfill data are reported by area instead of by program [i.e., Special Manufacturing Capability (SMC) and the Technical Support Facility (TSF) are reported under Test Area North (TAN)]. Future enhancements to the system will allow the landfill data to be reported by program to be consistent with all other reporting in INWMIS.

Because of organizational changes within various groups, the reporting for TAN also has changed. TAN is broken into three separate sections: TSF, the Containment Test Facility, and SMC. TSF and portions of the SMC are currently being reported into the INWMIS.

During 1994, a total of $77,036 \mathrm{~m}^{3}$ of solid industrial waste was disposed of in sanitary landfills at the INEL. This is an increase of $46.5 \%$ from the $52,568 \mathrm{~m}^{3}$ discarded in 1993 .
A total of $1,908,093 \mathrm{~kg}$ of liquid and airborne industrial waste was discharged in 1994 at the INEL. The industrial waste included $1,437,932$ $\mathrm{ks}$ of chemicals, $285,661 \mathrm{~kg}$ of total dissolved solids, and $184,500 \mathrm{~kg}$ of total hardness discharged as liquid wastes to the lithosphere at the INEL. This waste represents a $33.6 \%$ decrease over the total quantity of $2,872,675 \mathrm{~kg}$ in 1993 and a decrease from total quantity of $2,413,668$ $\mathrm{kg}$ in 1992.

In 1994, the INEL used a total of $15,067,581$ liters of fuel oil , which produced $182,534 \mathrm{~kg}$ of sulfur dioxide $\left(\mathrm{SO}_{2}\right)$ and $481 \mathrm{~kg}$ of particulates. During 1993, the INEL used 13,214,309 liters of fuel oil producing $128,514 \mathrm{~kg}$ of $\mathrm{SO}_{2}$ and $3,075 \mathrm{~kg}$ of particulates.

A total of 5,587,450,000 liters of water was pumped from the aquifer by the INEL during 1994, compared to $6,803,963,000$ liters in 1993 and 7,617,914,000 liters in 1992. However, the majority of this water returned to the lithosphere through seepage ponds. The remaining water was lost to the atmosphere through cooling towers and other evaporatory processes.

In October 1994, DOE awarded INEL's primary management and operating contract to Lockheed Idaho Technologies Company (LITCO). In order to report landfill data more accurately and to conform to LITCO's regulations, the INWMIS area and program codes were changed. However, these changes will not be indicated until the 1995 INWMIS Annual Report.

Please note that Argonne National LaboratoryWest did not operate its cooling towers in April, May, October, November, and December of 1994 due to routine reactor maintenance. 


\section{CONTENTS}

ABSTRACT $\ldots \ldots \ldots \ldots \ldots \ldots \ldots \ldots \ldots \ldots \ldots \ldots \ldots \ldots \ldots \ldots \ldots$

EXECUTIVE SUMMARY $\ldots \ldots \ldots \ldots \ldots \ldots \ldots \ldots \ldots \ldots \ldots \ldots$

ACRONYMS AND ABBREVIATIONS $\ldots \ldots \ldots \ldots \ldots \ldots \ldots \ldots \ldots \ldots$

CONVERSION FACTORS $\ldots \ldots \ldots \ldots \ldots \ldots \ldots \ldots \ldots \ldots \ldots \ldots \ldots$

DEFINITIONS $\ldots \ldots \ldots \ldots \ldots \ldots \ldots \ldots \ldots \ldots \ldots \ldots \ldots \ldots \ldots \ldots \ldots \ldots \ldots$

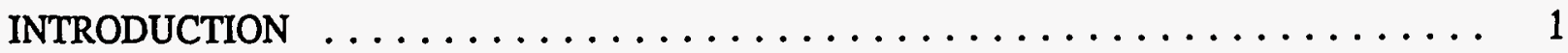

TAB SECTIONS

INEL Record to Date Summaries

INEL Record to Date Detail Summaries

INEL 1994 Year-to-Date Summaries

Argonne National Laboratory-West, 1994 Detail Data

Central Facilities Area, 1994 Detail Data

Chemical Processing Plant, 1994 Detail Data

Decontamination and Decommissioning, 1994 Detail Data

Naval Reactors Facility, 1994 Detail Data

Power Burst Facility, 1994 Detail Data

Radioactive Waste Management Complex, 1994 Detail Data

Special Manufacturing Capability, 1994 Detail Data

Test Area North, 1994 Detail Data

Test Reactor Area, 1994 Detail Data

Technical Support Facility-Test Area North, 1994 Detail Data

Water Reactor Research Test Facility-Test Area North, 1994 Detail Data 


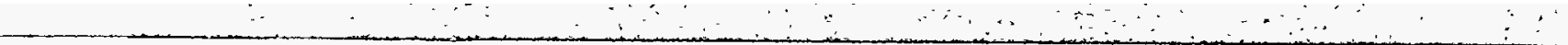




\section{ACRONYMS AND ABBREVIATIONS}

\begin{tabular}{|c|c|}
\hline ANL & Argonne National Laboratory-West \\
\hline CFA & Central Facilities Area \\
\hline PP & $\begin{array}{l}\text { Idaho Chemical Processing Plant } \\
\text { (same as ICPP) }\end{array}$ \\
\hline CTF & $\begin{array}{l}\text { Containment Test Facility (formerly } \\
\text { Loss-of-Fluid Test Facility) }\end{array}$ \\
\hline CY & calendar year \\
\hline HWSF & $\begin{array}{l}\text { Hazardous Waste Storage Facility } \\
\text { (same as CF-637) }\end{array}$ \\
\hline ICPP & $\begin{array}{l}\text { Idaho Chemical Processing Plant } \\
\text { (same as CPP) }\end{array}$ \\
\hline INEL & $\begin{array}{l}\text { Idaho National Engineering } \\
\text { Laboratory }\end{array}$ \\
\hline IRC & Idaho Research Center \\
\hline INWMIS & $\begin{array}{l}\text { INEL Nonradiological Waste } \\
\text { Management Information System }\end{array}$ \\
\hline IWMIS & $\begin{array}{l}\text { Industrial Waste Management } \\
\text { Information System }\end{array}$ \\
\hline $\mathrm{kg}$ & kilogram \\
\hline $\mathrm{L}$ & liter \\
\hline LOFT & $\begin{array}{l}\text { Loss-of-Fluid Test Facility (became } \\
\text { CTF on October 6, 1986) }\end{array}$ \\
\hline m & meter \\
\hline MWSF & $\begin{array}{l}\text { Mixed Waste Storage Facility } \\
\text { not available }\end{array}$ \\
\hline
\end{tabular}

$\begin{array}{ll}\text { NO }_{x} & \text { nitric oxides } \\ \text { NRF } & \text { Naval Reactors Facility } \\ \text { PBF } & \text { Power Burst Facility } \\ \text { RWMC } & \text { Radioactive Waste Management } \\ & \text { Complex (same as WMC) } \\ \text { SMC } & \text { Special Manufacturing Capability } \\ \text { SO } & \text { sulfur dioxide } \\ \text { TAN } & \text { Test Area North } \\ \text { TRA } & \text { Test Reactor Area } \\ \text { TSF } & \text { Technical Support Facility } \\ \text { WCB } & \text { Willow Creek Building } \\ \text { WDF } & \text { Waste Engineering Development } \\ & \text { Facility (same as WEDF) } \\ \text { WEDF } & \text { Waste Engineering Development } \\ & \text { Facility (same as WDF) } \\ \text { WER } & \text { Waste Experimental Reduction } \\ & \text { Facility (same as WERF) } \\ \text { WERF } & \text { Waste Experimental Reduction } \\ & \text { Facility (same as WER) } \\ \text { WMC } & \text { Radioactive Waste Management } \\ & \text { Complex (same as RWMC) } \\ \text { WRF } & \text { Water Reactor Research Test } \\ & \text { Facility (same as WRRTF) } \\ \text { WRRTF } & \text { Water Reactor Research Test } \\ & \text { Facility (same as WRF) } \\ & \end{array}$




\section{CONVERSION FACTORS}

\begin{tabular}{llr} 
To Convert & \multicolumn{1}{c}{ Into } & Multiply By \\
\cline { 3 - 3 } Cubic yards & Cubic meters & 0.7646 \\
Cubic meters & Cubic feet & 35.5140 \\
Liters & Gallons (U.S. liquid) & 0.2642 \\
Kilograms & Pounds & 2.2046
\end{tabular}

\section{DEFINITIONS}

Curie A unit of radioactivity, defined as that quantity of any radioactive nuclide that has $3.7 \mathrm{E}+10$ disintegrations per second (abbreviated $\mathrm{Ci}$ ).

Exponential notation Numbers in this document are expressed as either whole numbers or in exponential notation form. For example: $1,000,000=1 \times 10^{6}=1 \mathrm{E}+06$. 


\section{INEL NONRADIOLOGICAL WASTE MANAGEMENT INFORMATION FOR 1994 AND RECORD-TO-DATE}

\section{INTRODUCTION}

This report summarizes the nonradiological data for the Idaho National Engineering Laboratory (INEL) for 1994 and record-to-date. It includes summaries of the quantities of airborne and liquid effluents; the volumes of solid waste; the chemical usage; coal, fuel oil, and water used; and the quantities of $\mathrm{SO}_{2}$ and particulates released.

These data were taken from the INEL Nonradiological Waste Management Information System (INWMIS). The INWMIS is a computerized data base that replaced the Industrial Waste Management Information System (IWMIS). The INWMIS has been in place since October 1 , 1991 , and serves as the official record for all types of nonradiological waste stored, disposed, or shipped from the INEL.

This report provides graphics to aide in the presentation of the INEL summary data. The graphics display water, coal, chemical, fuel oil and diesel usage, and volumes of solid waste discarded at the landfill.

The INEL Industrial Waste Summary, a table that lists the waste data available for 1971 through 1994, is located within the tab section INEL Record-to-Date Summaries. Found in the tab section INEL Record-to-Date Detail Summaries are tables, bar graphs, and pie charts that summarize INEL record-to-date information and detailed data for solid and liquid industrial waste, sanitary landfill waste, airborne and liquid effluent waste, chemical usage, fuel oil usage, water usage, coal usage, and waste oil. The graphs only show data for 1985 through 1994.

Tables that summarize the INEL nonradiological waste information for the period January through December 1994 are located in the tab section INEL 1994 Year-To-Date Summaries. This section begins with tables that summarize both the solid waste and the airborne and liquid waste generated at each INEL facility.

\section{Reports Included in Document}

INEL Record to Date Summaries-These reports summarize all industrial waste by waste type located and used on the INEL for the period 1992 to 1994 and a cumulative total for the period 1971 to 1991.

INEL Record To Date Detail SummariesThese reports and graphs detail the record to date quantity and type of industrial waste for each INEL facility. This section also includes a record to date report (page INEL-10) of all airborne and liquid industrial waste substances for the period 1992 to 1994 and a cumulative total for the period 1971 to 1991 .

INEL 1994 Year-To-Date SummariesThese report summarize the year-to-date quantity of water pumped from the aquifer and discharged; the percent of waste accounted for at each facility; the quantity and type of industrial waste for each INEL facility; the types and quantities of fuel oil used at each INEL facility; and the gross volumes and weights of hazardous waste generated by facilities associated with the INEL.

Area 1994 Detail Data-These reports summarize data on both airborne and liquid disposed substances, industrial waste, water usage and disposal, fuel oil and diesel usage, coal usage, mixed waste, and hazardous waste generated at each facility. 
INEL Record-to-Date Summaries

INEL Industrial Waste Summary, Record to Date $\ldots \ldots \ldots \ldots \ldots \ldots \ldots \ldots$. . . . . . . . . .

INEL Industrial Usage Summary, Record to Date . . . . . . . . . . . . . . INEL-4 


\begin{tabular}{|c|c|c|c|c|c|c|}
\hline WASTE TYPE & & $1971-1991$ & 1992 & 1993 & 1994 & TOTAL \\
\hline $\begin{array}{l}\text { LANDFILL WASTE* } \\
\text { SOLID }\end{array}$ & (CUBIC METERS) & 674,009 & 51,433 & 52,568 & 77,036 & 855,046 \\
\hline OIL & (LITERS) & 133,912 & & & & 133,912 \\
\hline SOLVENTS & (LITERS) & 1,755 & & & & 1,755 \\
\hline $\begin{array}{l}\text { CHEMICALS } \\
\text { LIQUIDS } \\
\text { SOLIDS }\end{array}$ & $\begin{array}{l}\text { (LITERS) } \\
\text { (KILOGRAMS) } \\
\text { (CUBIC METERS) }\end{array}$ & $\begin{array}{r}132,247 \\
31,136 \\
689\end{array}$ & & & & $\begin{array}{r}132,247 \\
31,136 \\
689\end{array}$ \\
\hline $\begin{array}{l}\text { OTHERS } \\
\text { LIQUIDS } \\
\text { SOLIDS }\end{array}$ & $\begin{array}{l}\text { (LITERS) } \\
\text { (CUBIC METERS) }\end{array}$ & $\begin{array}{r}157,464 \\
223\end{array}$ & & & & $\begin{array}{r}157,464 \\
223\end{array}$ \\
\hline LIQUID EFFLUENT & (KILOGRAMS) & $73,456,092$ & $2,405,973$ & $2,457,195$ & $1,907,906$ & $80,227,167$ \\
\hline $\begin{array}{l}\text { AIRBORNE EFFLUENT } \\
\text { CHEMICALS } \\
\text { FUEL OIL AND DIESEL }\end{array}$ & (KILOGRAMS) & $5,236,644$ & 7,694 & 415,480 & 187 & $5,660,005$ \\
\hline $\begin{array}{l}\text { S02 } \\
\text { PARTICULATE }\end{array}$ & $\begin{array}{l}\text { (KILOGRAMS) } \\
\text { (KILOGRAMS) }\end{array}$ & $\begin{array}{r}6,896,468 \\
441,389\end{array}$ & $\begin{array}{r}84,861 \\
2,481\end{array}$ & $\begin{array}{r}128,514 \\
3,075\end{array}$ & $\begin{array}{r}182,534 \\
481\end{array}$ & $\begin{array}{r}7,292,377 \\
447,427\end{array}$ \\
\hline $\begin{array}{r}\text { COAL } \\
\text { SO2 } \\
\text { NO2 }\end{array}$ & $\begin{array}{l}\text { (KILOGRAMS) } \\
\text { (KILOGRAMS) }\end{array}$ & $\begin{array}{r}77 \\
458\end{array}$ & $\begin{array}{r}21 \\
109\end{array}$ & $\begin{array}{l}10 \\
96\end{array}$ & $\begin{array}{r}4 \\
57\end{array}$ & $\begin{array}{l}112 \\
720\end{array}$ \\
\hline WASTE OIL & (LITERS) & $1,283,361$ & 53,367 & 51,531 & 12,011 & $1,400,269$ \\
\hline
\end{tabular}

- NOTE: DOES INCLUDE LANDFILL WASTE FOR WHICH ONLY A WEIGHT IS AVAILABLE

- NOTE: SINCE APRIL 1984, THE DEPARTMENT OF ENERGY'S POSITION HAS BEEN TO ENSURE THAT ALL HAZARDOUS WASTE IS PROPERLY MANAGED UNDER TIE RESOURCE CONSERVATION AND RECOVERY ACT (RCRA). AVAILABLE INFORMATION ASSOCIATED WITH THE CHEMICALS DISPOSED OF IN THE LANDFILL SINCE 1984 DOES NOT INDICATE THAT THEY WERE HAZARDOUS AS DEFINED UNDER RCRA.

* NOTE: BASED ON AN EXTENSIVE REVIEW OF EMISSIONS DATA, CORRECTIONS TO SO2 EMITTED DATA HAVE BEEN PERFORMED. 
SCHED. NO. INRPTOZOA

INEL INDUSTRIAL USAGE SUMMARY

RECORD TO DATE

\begin{tabular}{|c|c|c|c|c|c|c|}
\hline WASTE TYPE & & $1971-1991$ & 1992 & 1993 & 1994 & TOTAL \\
\hline FUEL OIL \& DIESEL & (LITERS) & $402,532,798$ & $9,586,173$ & $13,214,309$ & $15,067,581$ & $440,400,862$ \\
\hline COAL & (TONS) & 55,342 & 12,464 & 11,635 & 8,723 & 88,164 \\
\hline WATER PUMPED & (1000 LITERS) & $177,250,734$ & $7,617,914$ & $6,803,963$ & $5,587,450$ & $197,260,060$ \\
\hline
\end{tabular}




\section{INEL Record-to-Date Detail Summaries}

INEL Solid Landfill Waste In Cubic Meters Summary,

Record to Date .............................. INEL-7

INEL Liquid Landfill Waste In Liters, Record to Date . . . . . . . . . . . . . INEL-8

CY 1985-1994 INEL Sanitary Landfill Waste,

Bar Graph and Pie Charts . . . . . . . . . . . . . . . . . . INEL-9

Airborne And Liquid Industrial Waste Substances

Summary In Kilograms, Record To Date . . . . . . . . . . . . . . . INEL-10

CY 1985-1994 INEL Chemical Usage (Released As

Airborne Effluents), Bar Graph and Pie Chart .................. INEL-15

CY 1985-1994 INEL Chemical Usage Released As

Liquid Effluents), Bar Graph and Pie Chart . . . . . . . . . . . . . INEL-16

INEL Fuel Oil Diesel Summary, Record to Date . . . . . . . . . . . . . . . INEL-17

CY 1985-1994 INEL Fuel Oil and Diesel Usage,

Bar Graph and Pie Chart . . . . . . . . . . . . . . . . . . INEL-19

CY 1985-1994 Sulfur Dioxide Emitted From INEL Fuel

Oil and Diesel Usage, Bar Graph and Pie Chart . . . . . . . . . . . . . . . INEL-20

CY 1985-1994 Particulate Emitted From INEL Fuel

Oil and Diesel Usage, Bar Graph and Pie Chart . . . . . . . . . . . . . . . INEL-21

INEL Water Usage Summary In Thousand Liters, Record to Date . . . . . . . . . . INEL-22

INEL Water Pumped Summary In Thousand Liters, Record to Date . . . . . . . . . INEL-23

CY 1985-1994 INEL Water Usage, Bar Graph and Pie Chart . . . . . . . . . . . . INEL-24

CY 1994 INEL Water Usage, Pie Charts . . . . . . . . . . . . . . . INEL-25

INEL Coal Summary, Record to Date . . . . . . . . . . . . . . . INEL-26

CY 1987-1994 INEL Coal Usage, Bar Graph . . . . . . . . . . . . . . INEL-28

CY 1987-1994 Sulfur Dioxide Emitted From

INEL Coal Usage, Bar Graph . . . . . . . . . . . . . . . . . . INEL-29

CY 1987-1994 Nitric Oxide Emitted From

INEL Coal Usage, Bar Graph $\ldots \ldots \ldots \ldots \ldots \ldots \ldots \ldots \ldots \ldots \ldots \ldots \ldots$ INEL-30

INEL Waste Oil Summary In Liters, Record to Date . . . . . . . . . . . . . . INEL-31 


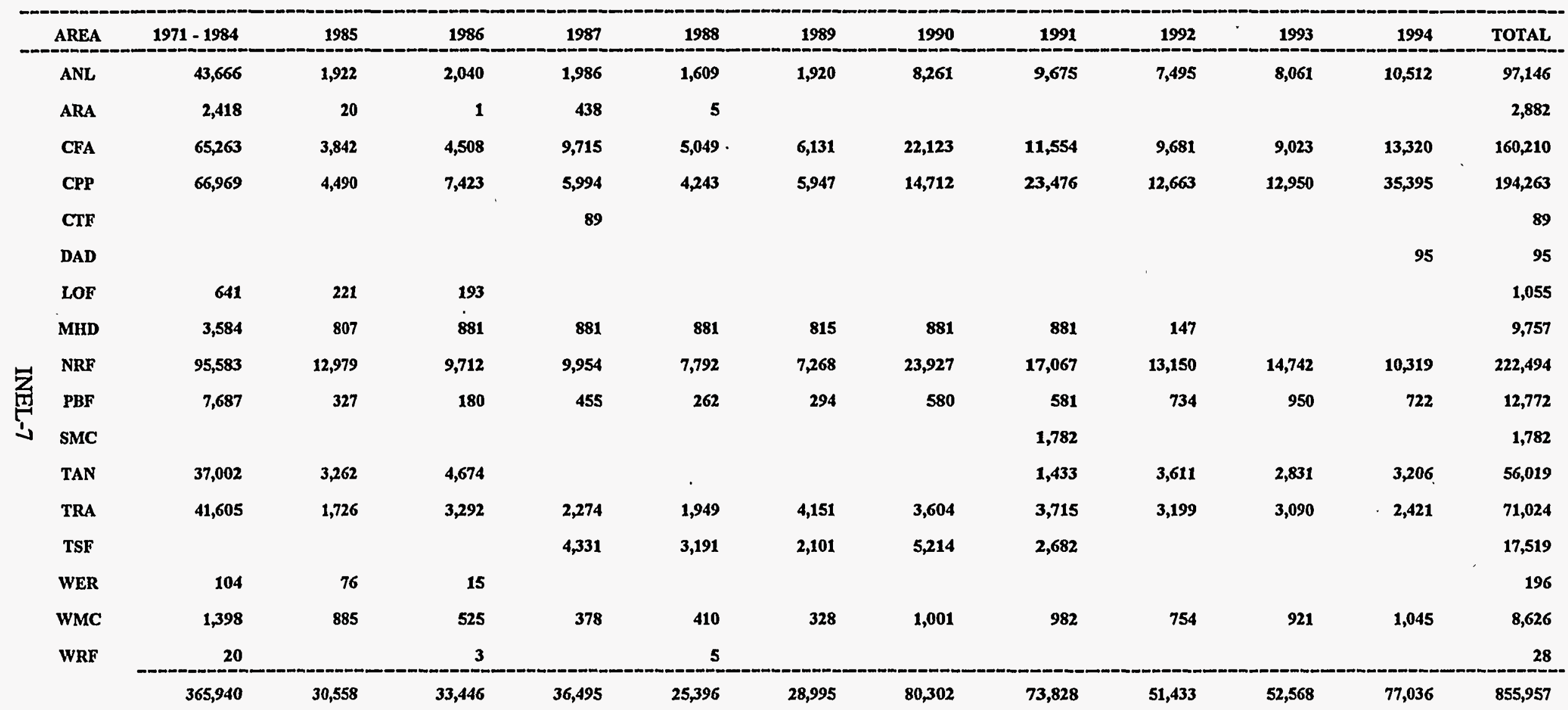

NOTE: DOES NOT INCLUDE LANDFILL WASTE FOR WHICH ONLY A WEIGHT IS AVAILABLE THIS COMPRISES 31,136 KILOGRAMS FOR THE YEARS 1971 - 1988.

DETAILS MAY NOT ADD UP TO TOTALS BECAUSE OF ROUNDING $O$ IN A COLUMN INDICATES A TOTAL OF $<0.5$ 
SCHED. NO. INRPT035A

INEL LIQUID LANDFILC WASTE IN LITERS

RECORD TO DATE

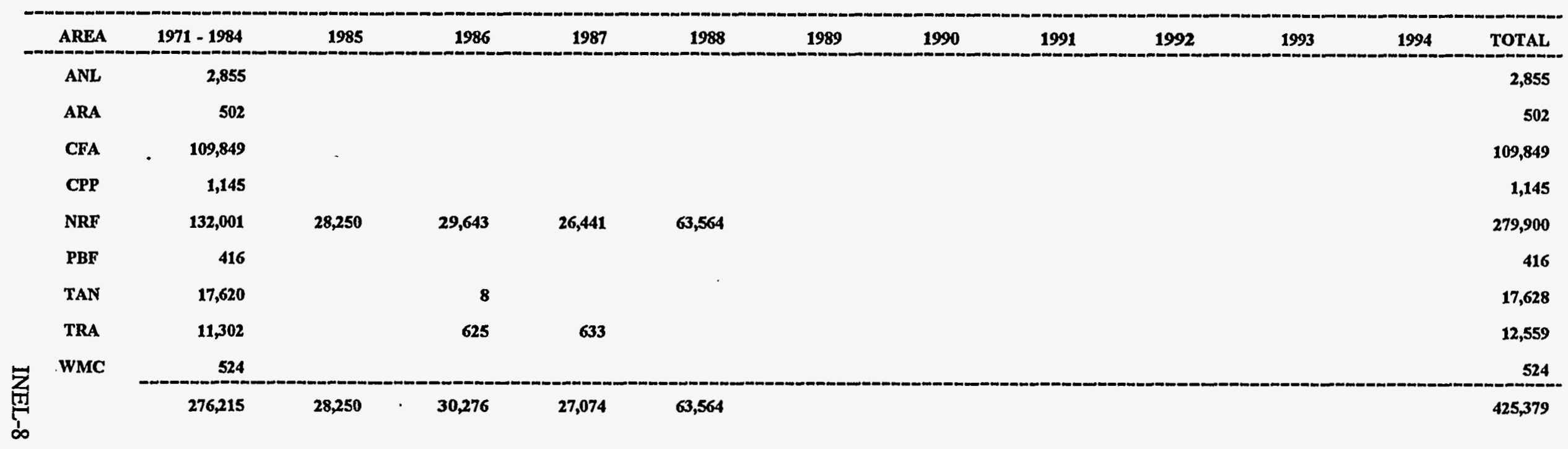

NOTE: INCLUDES ALL LANDFILL WASTE DENOTED BY LITERS ON THE INEL INDUSTRIAL WASTE SUMMARY REPORT

0 IN A COLUMN INDICATES A TOTAL OF $<0.5$ 


\section{CY 1985 - 1994 INEL SANITARY LANDFILL WASTE}

CY 1994 INEL WASTE BY TYPE
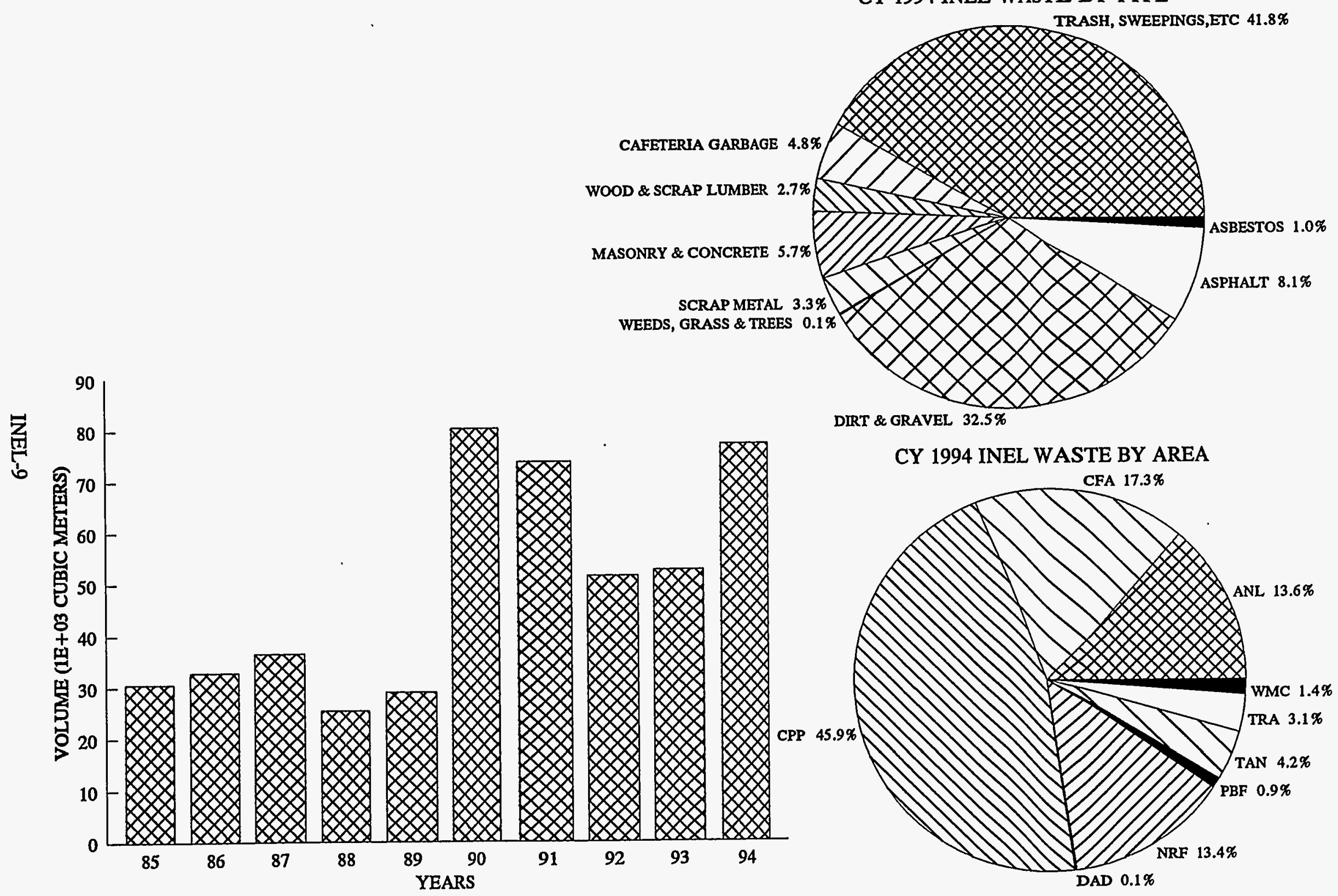

DIRT \& GRAVEL $32.5 \%$

CY 1994 INEL WASTE BY AREA

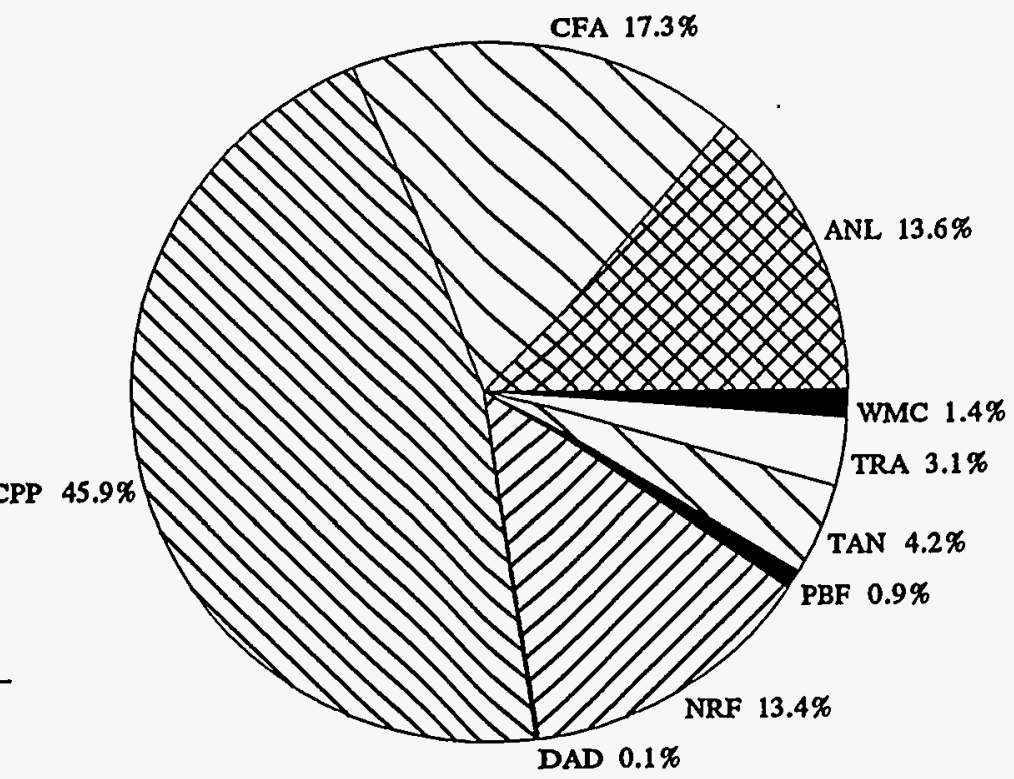


SCHED. NO. INRPT036

AIRBORNE AND LIQUID

INDUSTRIAL HASTE SUBSTANCES SUMMARY IN KILOGRAMS

RECORD TO DATE

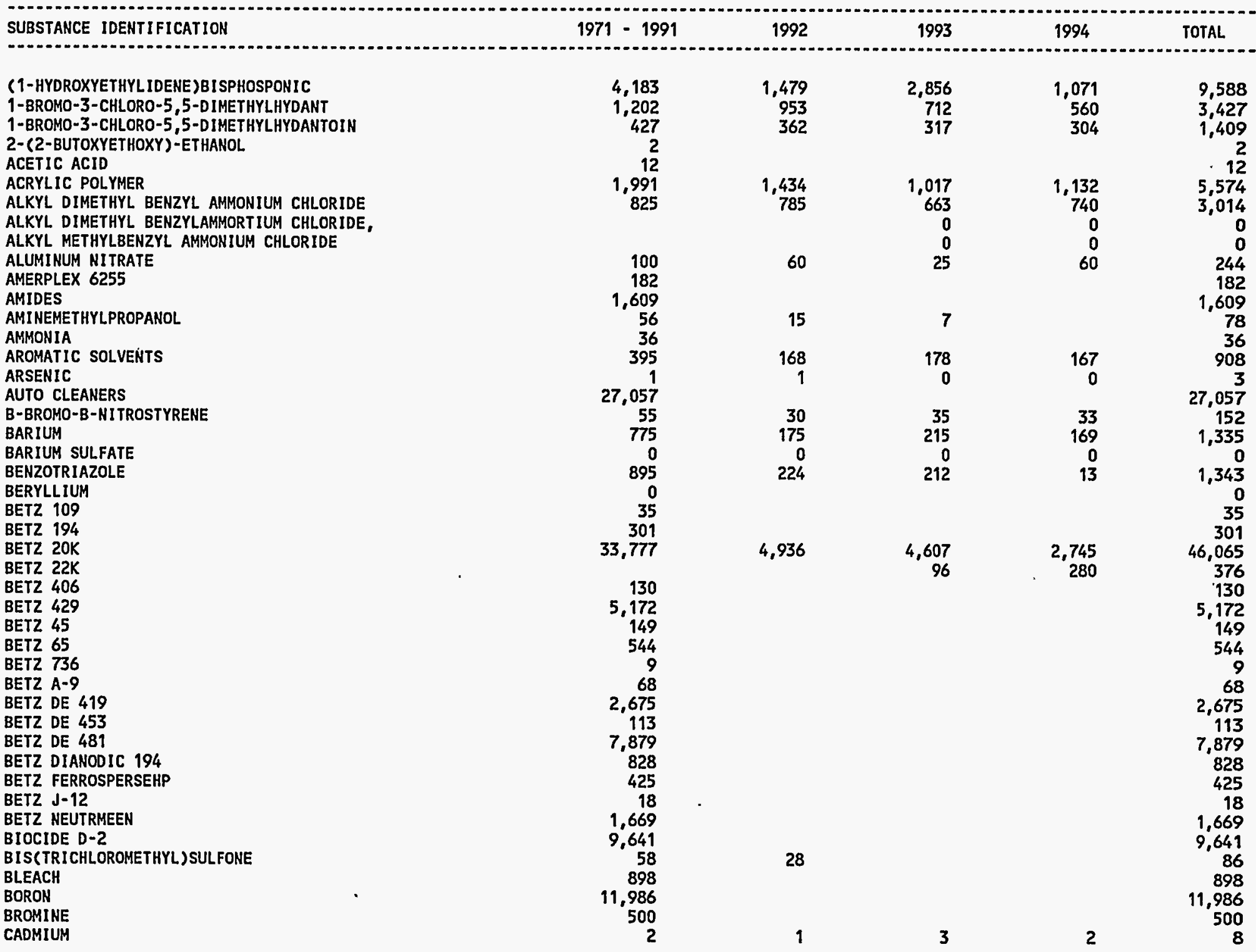

DETAILS MAY NOT ADD UP TO TOTALS BECAUSE OF ROUNDING

$O$ IN A COLUMN INDICATES A TOTAL OF $<0.5$ 
AIRBORNE AND LIQUID

INDUSTRIAL WASTE SUBSTANCES SUMMARY IN KILOGRAMS

RECORD TO DATE

SUBSTANCE IDENTIFICATION

\section{CALCIUM CHLORIDE}

CALCIUM FLUORIDE

CALCIUM ION

CALCIUM NITRATE

CALCIUM PERCHLORATE

CALCIUM PHOSPHATE, TRIBASIC

CALCIUM SULFATE, ANHYDROUS

CARBOHYDRAZIDE

CARBONTHIOCYANATES

CARBOXYALKYLATED POLYSACCHARIDE

CARBOXYLATED LIGNIN

CHLORIDE ION

CHLORINE

CHLOROCARBONS

CHLOROHYDROCARBONS

CHLOROSULFONES

CHROMATE ION

CHROMIUM

CITRIC ACID

量

CLOROHYDROCARBONS

COPPER

CYCLOHEXYLAMINE

DIANODIC 194

DIETHYLAMINOETHANOL

DIMETHYLAMINE

DIMETHYLFORMAMIDE(DMF)

DIMETHYLISOPROPANOLAMINE

DIPHOSPHONATE

DISPERSANT 403

DODECYLGUANIDINE HYDROCHLORIDE

DREWGARD

EDETATE SODIUM

ETHYL ALCOHOL

ETHYLAMINE

TTHYLEEDIAMINE TETRAACETIC ACID

FLUORIDE ION

FORMIC ACID

HEAVY AROMATIC NAPTHA

HEDP

HEXAVALENT CR

HPA

HPS I CARBOXAL ACID

HYDRAZINE

HYDROCARBON-AMINES
1971 - 1991

1992

1993

1994

TOTAL

218
111
26,028
166
7
47
2

131
50
691
26
4
28
1

54
21
253
19
2
12
0
8

283

$\begin{array}{rrrrr}283 & 1 & 1 & 1 & 283 \\ 10,326,348 & 0 & 0 & 0 & 3 \\ 21 & 722,131 & 827,334 & 598,793 & 12,474,606 \\ 2 & & & & 21 \\ 472 & & & & 2 \\ 1,263 & & & & 472 \\ 327 & & 6 & 7 & 1,263 \\ 120 & 9 & & & 327 \\ 91 & & & & 91 \\ 0 & & & & 0\end{array}$

1,815

1,471

15,531

2,131

2,131
56
13,249

13,026

510
116

10

21

212
20

580
15,007

5

42
11,193

11,193
2,631

2,631
4,415

16
47
47
203
104

24
768

16
471
47
203
2
104
482
21

10

131

50

94

45
28
1

21

16

398
3

3

145
1

136
561

21

4
20

3
3

533

27. 066

256

18

116
4

283

3

2

263

327
91
91
0

815

1,523
15,531

15,531
147

2,131
24

$\begin{array}{r}24 \\ 85 \\ \hline\end{array}$

13,249

13,026

1,826

172
27

736

24
921

921
6,385

5
62

11,201

2,631

4,415

12

12
24
768

DETAILS MAY NOT ADD UP TO TOTALS BECAUSE OF ROUNDING

0 IN A COLUMN INDICATES A TOTAL OF $<0.5$ 
SCHED. NO. INRPT036

AIRBORNE AND LIQUID

INDUSTRIAL WASTE SUBSTANCES SUMMARY IN KILOGRAMS

RECORD TO DATE

\begin{tabular}{|c|c|c|c|c|c|}
\hline SUBSTANCE IDENTIFICATION & $1971-1991$ & 1992 & 1993 & 1994 & TOTAL \\
\hline HYDROCARBONS & 2,830 & & & & 2,830 \\
\hline HYDROGEN CHLORIDE & 114 & 52 & & & 167 \\
\hline HYDROXYCARBONS & 18,910 & & 9 & 2 & 18,921 \\
\hline HYPOCHLORITE & 16,053 & & & & 16,053 \\
\hline HYPOCHLORITE ION & 148,629 & 1,776 & 650 & 242 & 151,296 \\
\hline $\begin{array}{l}\text { IMPURITY } \\
\text { IRON }\end{array}$ & 22 & 19 & 17 & 16 & 74 \\
\hline ISOPROPANOL & 57 & 26 & & & 83 \\
\hline $\begin{array}{l}\text { I SOPROPYL ALCOHOL } \\
\text { JANITORAL SUPPIIES }\end{array}$ & 170 & 157 & 133 & 149 & 609 \\
\hline $\begin{array}{l}\text { JANITORAL SUPPLIES } \\
\text { JANITORIAL, SUPPLIES }\end{array}$ & $\begin{array}{r}938 \\
7.446\end{array}$ & 8.762 & & & \\
\hline $\begin{array}{l}\text { JANITORIAL SUPPLIES } \\
\text { KP40 COOLANT CNTRL }\end{array}$ & $\begin{array}{r}7,446 \\
73\end{array}$ & 8,102 & 9,875 & 7,510 & $\begin{array}{r}33,593 \\
73\end{array}$ \\
\hline KWIK KLEEN & 2,098 & & & & 2,098 \\
\hline LAUNDRY PRODUCTS & 96,483 & 3,516 & 1,153 & & 101,153 \\
\hline $\begin{array}{l}\text { LEAD } \\
\end{array}$ & 243 & & & 5 & 248 \\
\hline $\begin{array}{l}\text { LIGNOSULFONIC ACID, SODIUM SALT } \\
\text { M-60 ALGAECIDE }\end{array}$ & 4 & & & 27 & $\begin{array}{r}27 \\
4\end{array}$ \\
\hline $\begin{array}{l}\text { M-80T ALGAECIDE } \\
\text { MANGANESE }\end{array}$ & 4 & & & & 4 \\
\hline MERCURY & 64 & & & & 64 \\
\hline METHBISTHIOCYANATE & 68 & & & & 68 \\
\hline $\begin{array}{l}\text { METHYLENE BIS(THIOCYANATE) } \\
\text { MOGUL PC } 1222\end{array}$ & $\begin{array}{r}208 \\
8,612\end{array}$ & 176 & 148 & 164 & $\begin{array}{r}697 \\
8,612\end{array}$ \\
\hline MOLYBDATE SALT & 1 & & 0 & 0 & 1 \\
\hline MORPHOLINE & 1,835 & 8 & 10 & 8 & 1,862 \\
\hline N-HYDROCARBON & 10,024 & & 44 & 9 & 10,077 \\
\hline N-METHYLPYRROLIDONE & 109 & 75 & 54 & 47 & 284 \\
\hline N/A & & & 0 & 0 & 0 \\
\hline NALCO 7312 & 178 & & & & 178 \\
\hline NALCO 7323 & 185 & & & & 185 \\
\hline NALCOOL 7351 & 840 & & & & 840 \\
\hline NAPTHA & 39 & & & & 39 \\
\hline NITRATE ION & $1,562,706$ & 39,847 & 13,963 & 12,198 & $1,628,714$ \\
\hline NITRIC OXIDE & 546,325 & 765 & 73,306 & 0 & 620,396 \\
\hline NITRILOTRIACETIC ACID & 119 & & & & 119 \\
\hline NITRITE ION & 3 & & & & \\
\hline NITRITE SALT & 1 & & 0 & 0 & 2 \\
\hline NITROGEN DIOXIDE & $4,685,021$ & 6,881 & 341,935 & 0 & $5,033,837$ \\
\hline NONYLPHENOXYPOLY(ETHYLENEOXY)ETHANO & 27 & & 3 & 1 & 31 \\
\hline NONYLPHENOXYPOLY(ETHYLENEOXY)ETHANOL & 16 & 21 & 13 & 13 & 64 \\
\hline $\begin{array}{l}\text { OROCOL } 194 \\
\text { OROCOL } 734\end{array}$ & $\begin{array}{r}68 \\
70,312\end{array}$ & & & & $\begin{array}{r}68 \\
70.312\end{array}$ \\
\hline ORTHO PHOSPHATE & & & & 9 & 0,0 \\
\hline OXYCARBONS & 0 & & & & \\
\hline PHOSPHATE & 29,654 & & 8 & 4 & 29,666 \\
\hline
\end{tabular}

DETAILS MAY NOT ADD UP TO TOTALS BECAUSE OF ROUNDING

$O$ IN A COLUMN INDICATES A TOTAL OF $<0.5$ 

RECORD TO DATE

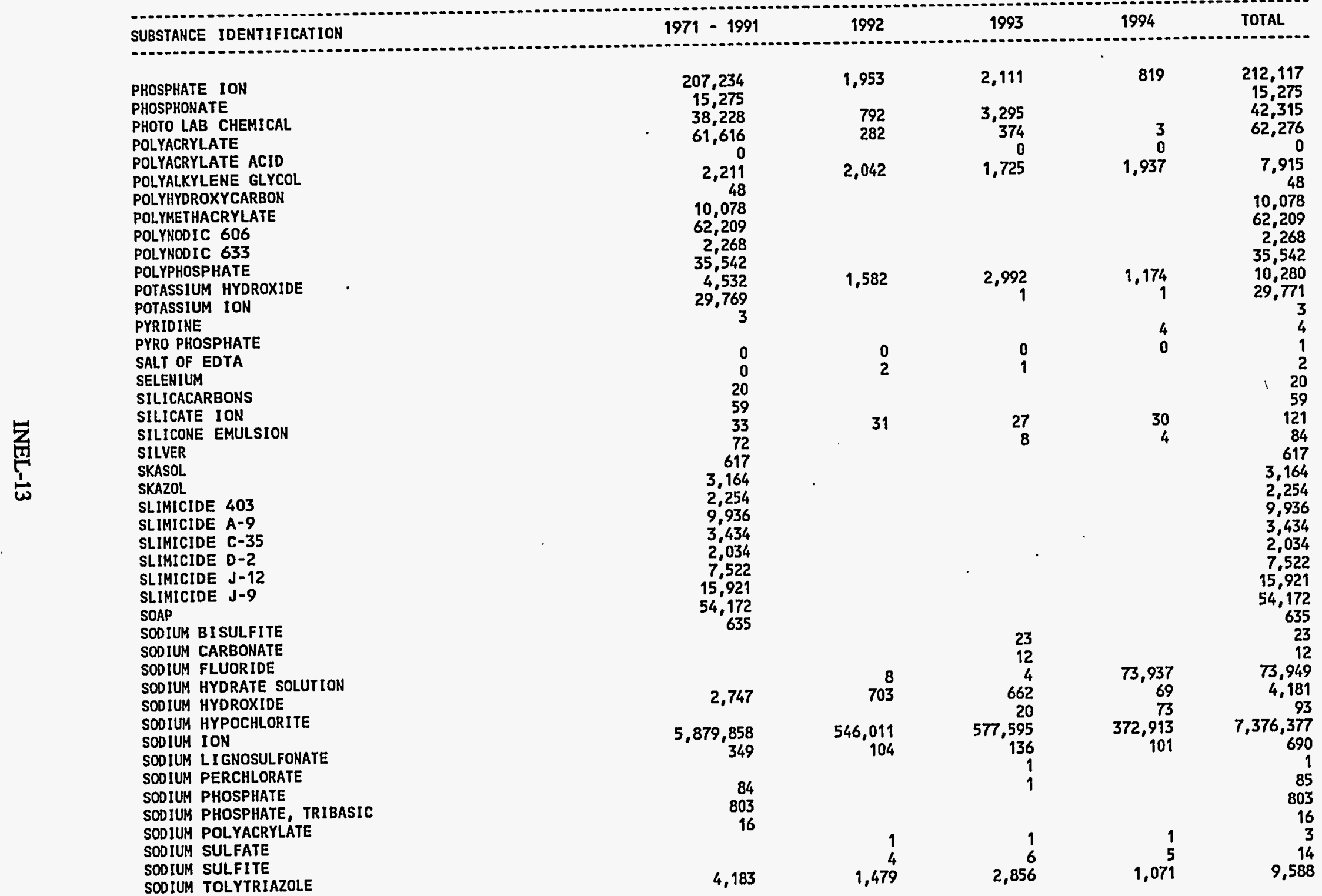

DETAILS MAY NOT ADD UP TO TOTALS BECAUSE OF ROUNDING

0 IN A COLUMN INDICATES A TOTAL OF $<0.5$ 
SCHED. NO. INRPT036

AIRBORNE AND LIQUID

INDUSTRIAL WASTE SUBSTANCES SUMMARY IN KILOGRAMS

RECORD TO DATE

SUBSTANCE IDENTIFICATION

1971 - 1991

1992

1993

1994 TOTAL

\section{SODIUM TRIPOLYPHOSPHATE}

SPRA-SAF

STODDARD SOLVENT

SULFATE ION

SULFITE ION

SULFONATE

SULFUR DIOXIDE

SULFURIC ACID

TOS

TETRAPOTASSIUM PYROPHOSPHATE

THIOCARBONS

THIOHYDROCARBONS

THIOUREA

TOLYTRIAZOLE

TOTAL. HARDNESS

TRIBUTYLTIN OXIDE

TRIVALENT CR

ZINC ION

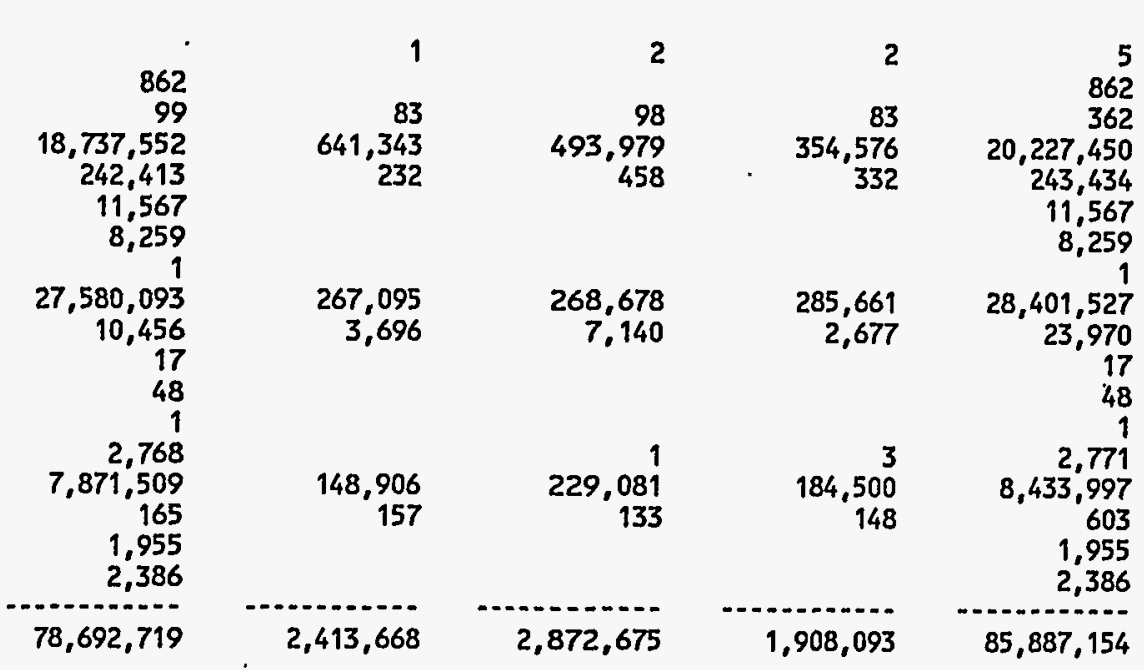

0 IN A COLUMN INDICATES A TOTAL OF $<0.5$ 


\section{CY 1985 - 1994 INEL CHEMICAL USAGE (RELEASED AS AIRBORNE EFFLUENTS)}

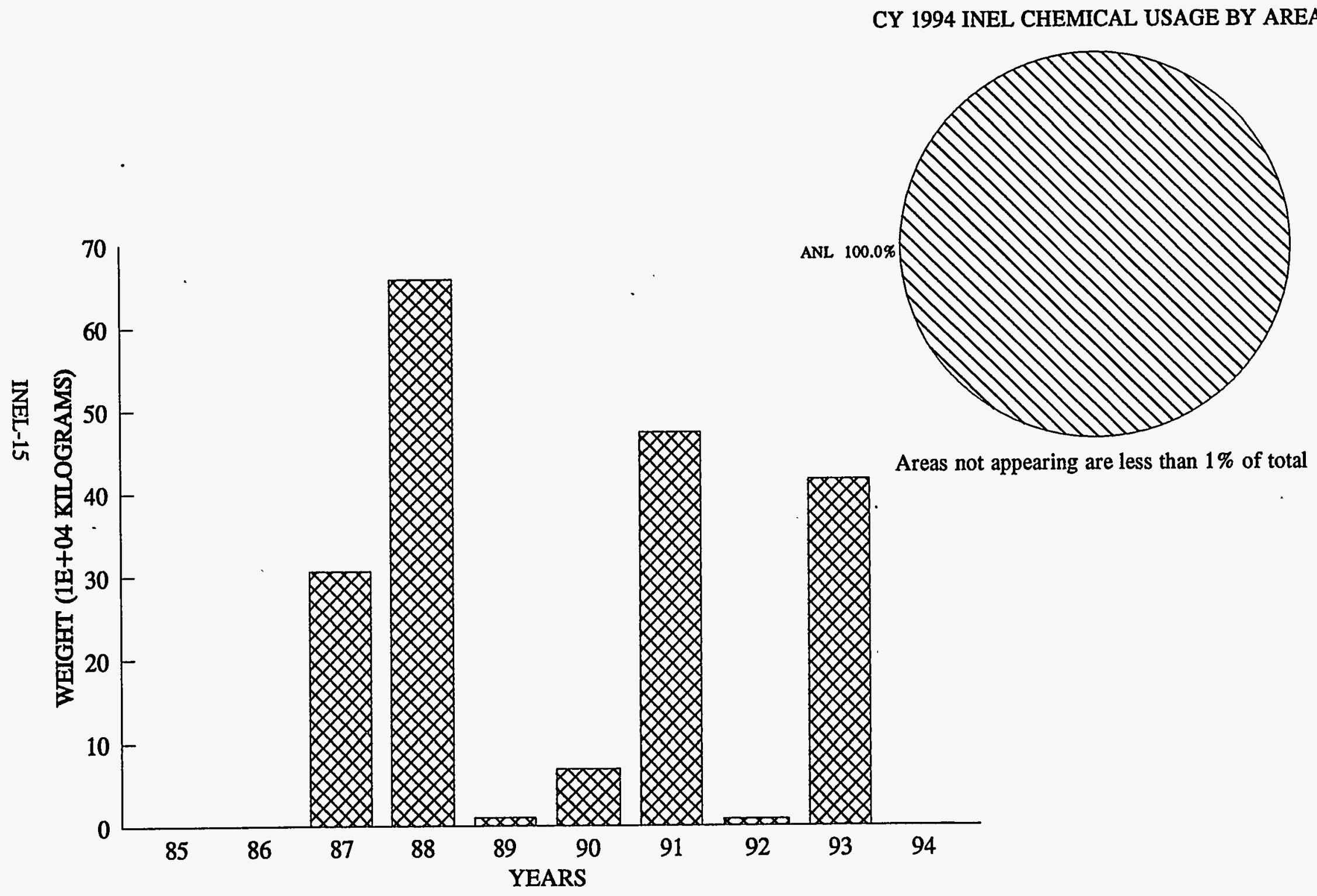




\section{CY 1985 - 1994 INEL CHEMICAL USAGE (RELEASED AS LIQUID EFFLUENTS)}

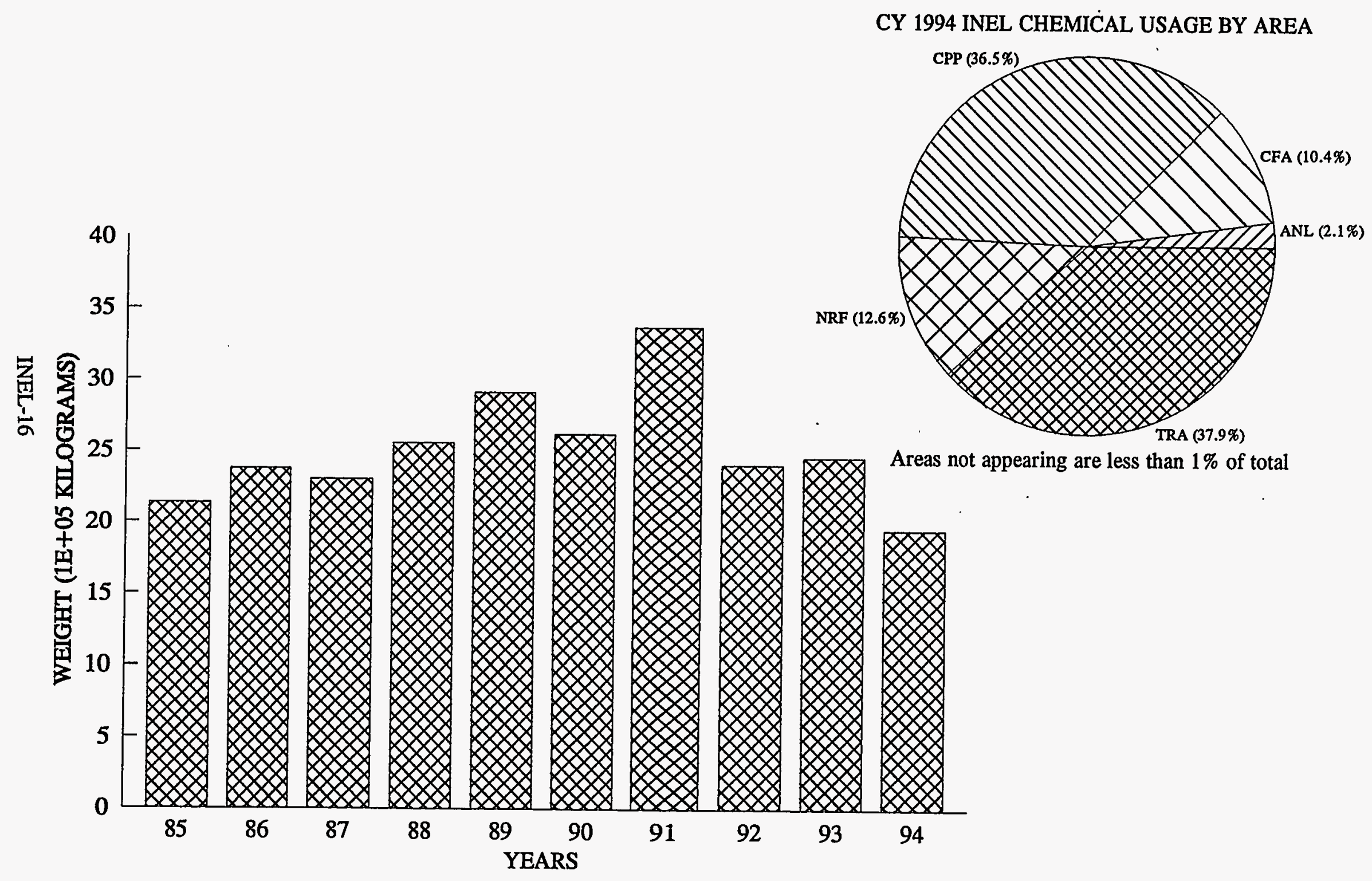


RECORD TO DATE

\begin{tabular}{|c|c|c|c|c|c|c|c|c|c|c|c|c|}
\hline \multirow[b]{2}{*}{ AREA } & \multicolumn{4}{|c|}{$1971-1992$} & \multicolumn{4}{|c|}{1993} & \multicolumn{4}{|c|}{1994} \\
\hline & $\begin{array}{l}\text { FUEL OIL } \\
\text { (LITERS) }\end{array}$ & $\begin{array}{l}\mathrm{SO2} \\
\text { (KG) }\end{array}$ & $\begin{array}{l}\text { PART. } \\
\text { (KG) }\end{array}$ & $\begin{array}{l}\text { DIESEL } \\
\text { (LITERS) }\end{array}$ & $\begin{array}{l}\text { FUEL OIL } \\
\text { (LITERS) }\end{array}$ & $\begin{array}{l}\text { SO2 } \\
\text { (KG) }\end{array}$ & $\begin{array}{l}\text { PART . } \\
\text { (KG) }\end{array}$ & $\begin{array}{l}\text { DIESEL } \\
\text { (LITERS) }\end{array}$ & $\begin{array}{l}\text { FUEL OIL } \\
\text { (LITERS) }\end{array}$ & $\begin{array}{l}\mathrm{SO2} \\
\text { (KG) }\end{array}$ & $\begin{array}{l}\text { PART. } \\
\text { (KG) }\end{array}$ & $\begin{array}{l}\text { DIESEL } \\
\text { (LITERS) }\end{array}$ \\
\hline ANL & $38,028,954$ & 281,378 & 41,725 & & $2,333,143$ & 12,817 & 3,075 & & $2,267,330$ & 14,462 & 481 & 1,092 \\
\hline ARA & $1,878,406$ & 13,212 & 523 & 56,119 & & & & & & & & \\
\hline CFA & $27,330,418$ & 266,179 & 22,262 & $2,019,477$ & 587,945 & 4,934 & & 232,461 & 398,357 & 2,895 & & 92,523 \\
\hline CPP & $99,472,223$ & $1,368,252$ & 5,504 & & $2,657,427$ & 44,210 & & & $4,251,973$ & 70,738 & & \\
\hline CTF & 620,105 & 4,116 & 0 & 87,587 & & & & & & & & \\
\hline LOF & $1,508,115$ & 10,490 & 3 & 270,690 & & & & & & & & \\
\hline MHD & $2,629,180$ & 17,903 & 0 & & & & & & & & & \\
\hline$Z^{N R F}$ & $94,952,141$ & $2,470,928$ & 155,127 & & $2,561,152$ & 39,257 & & & $1,997,210$ & 59,582 & & \\
\hline PBF & $2,765,116$ & 17,846 & 231 & 545,540 & 55,691 & 315 & & & 60,097 & 340 & & \\
\hline$\exists$ sMc & $1,113,536$ & 6,146 & & & $2,085,377$ & 10,703 & & & $1,728,152$ & 8,870 & & \\
\hline TAN & $39,629,913$ & 861,640 & 36,306 & 155,709 & & & & & & & & \\
\hline TRA & $47,513,788$ & $1,456,415$ & 182,189 & $35,205,458 *$ & & 7,165 & & $1,038,097^{*}$ & 945,256 & 6,524 & & \\
\hline TSF & $10,265,316$ & 178,414 & 0 & $1,052,640$ & $1,492,825$ & 8,455 & & & $2,918,877$ & 16,820 & & \\
\hline HMC & 443,055 & 2,820 & 0 & 28,493 & & & & & & & & \\
\hline WRF & $4,491,521$ & 25,590 & 0 & 55,475 & 170,192 & 657 & & & 406,715 & 2,303 & & \\
\hline Tota & $372,641,785$ & $6,981,329$ & 443,870 & $39,477,187$ & $11,943,751$ & 128,514 & 3,075 & $1,270,558$ & $14,973,966$ & 182,534 & 481 & 93,614 \\
\hline
\end{tabular}

* tra emergency generator 
SCHED. NO. INRPT031

INEL. FUEL OIL \& DIESEL. SUMMARY

RECORD TO DATE (CONTINUED)

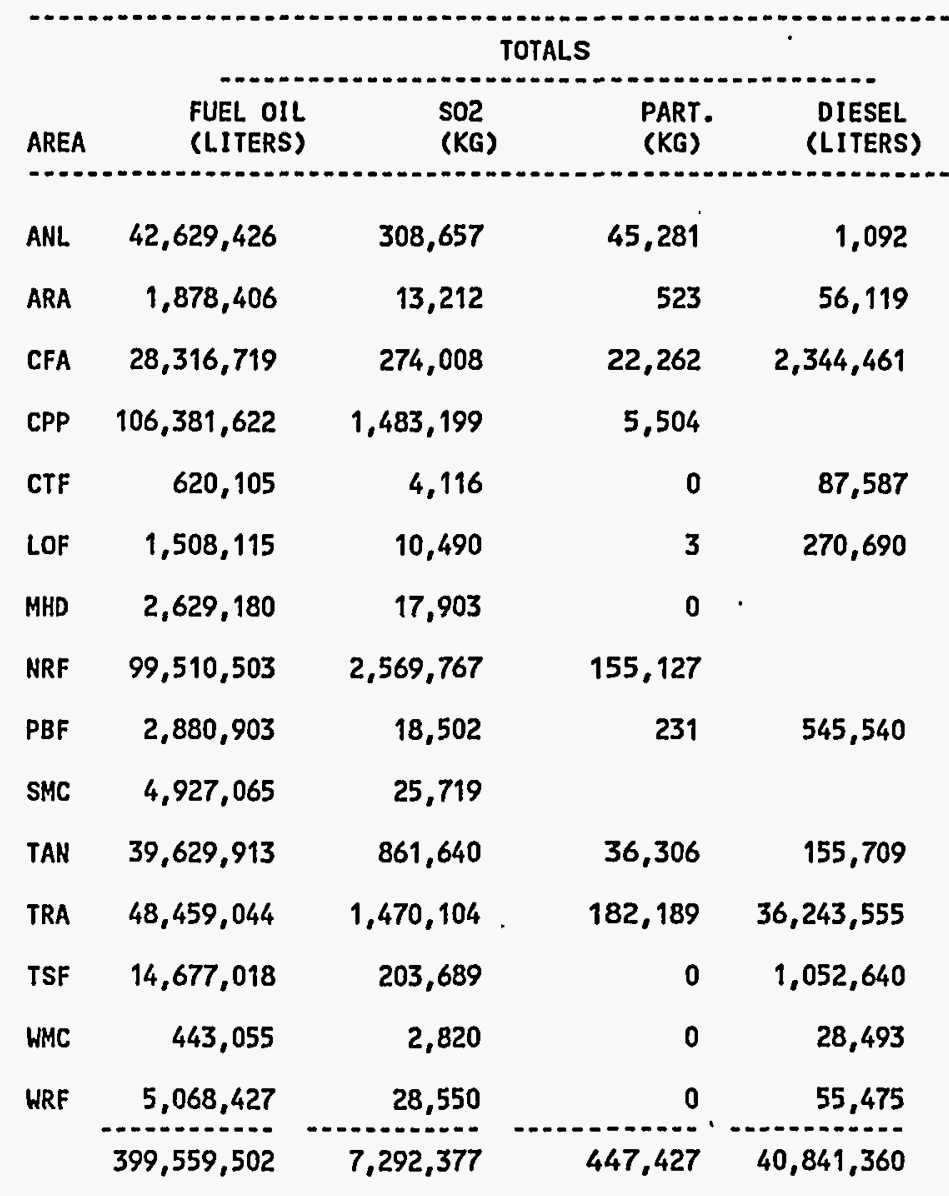




\section{CY 1985 - 1994 INEL FUEL OIL \& DIESEL USAGE}

CY 1994 INEL FUEL OIL \& DIESEL USAGE BY AREA

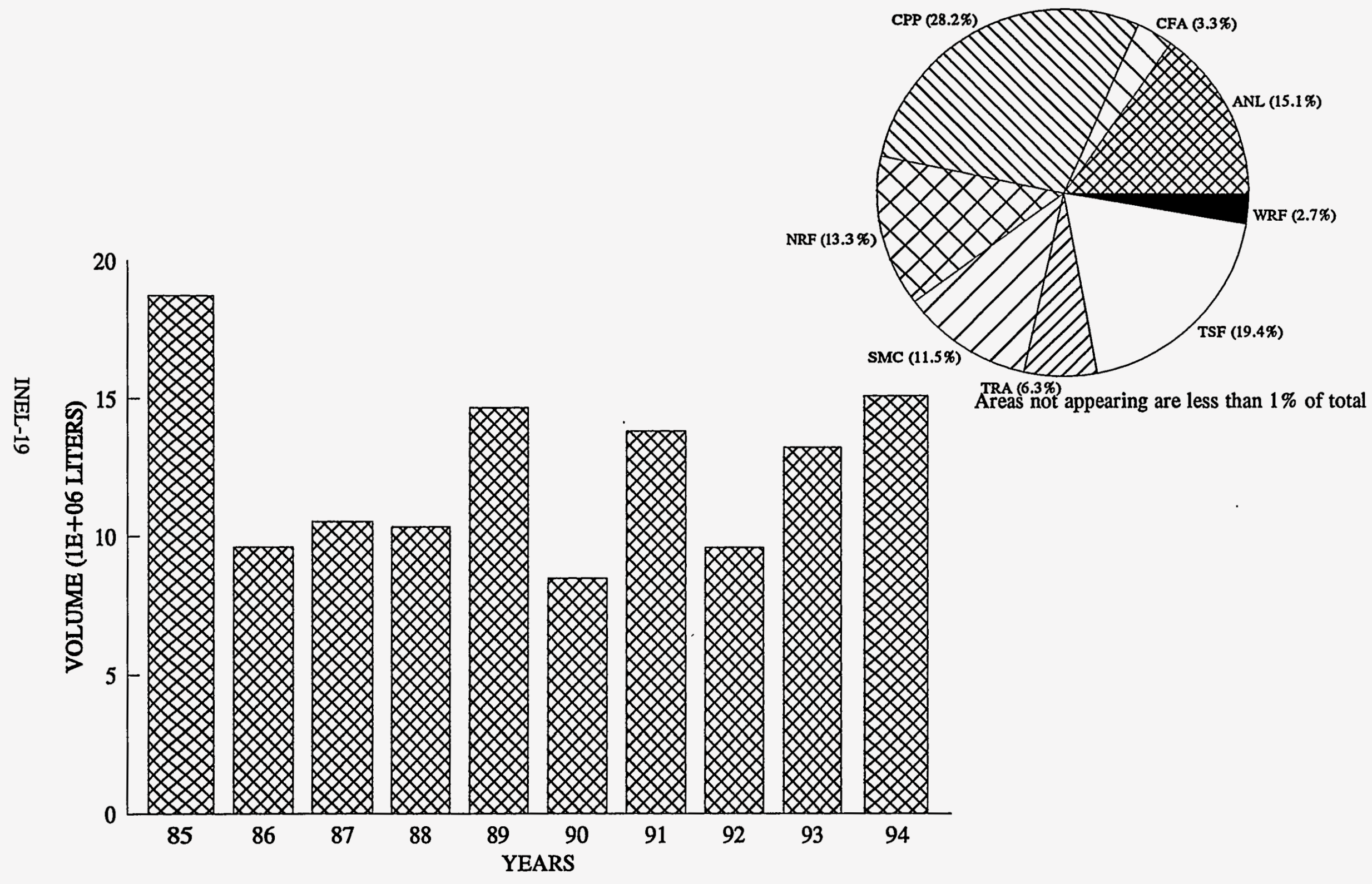




\section{CY 1985 - 1994 SULFUR DIOXIDE EMITTED FROM INEL FUEL OIL \& DIESEL USAGE}

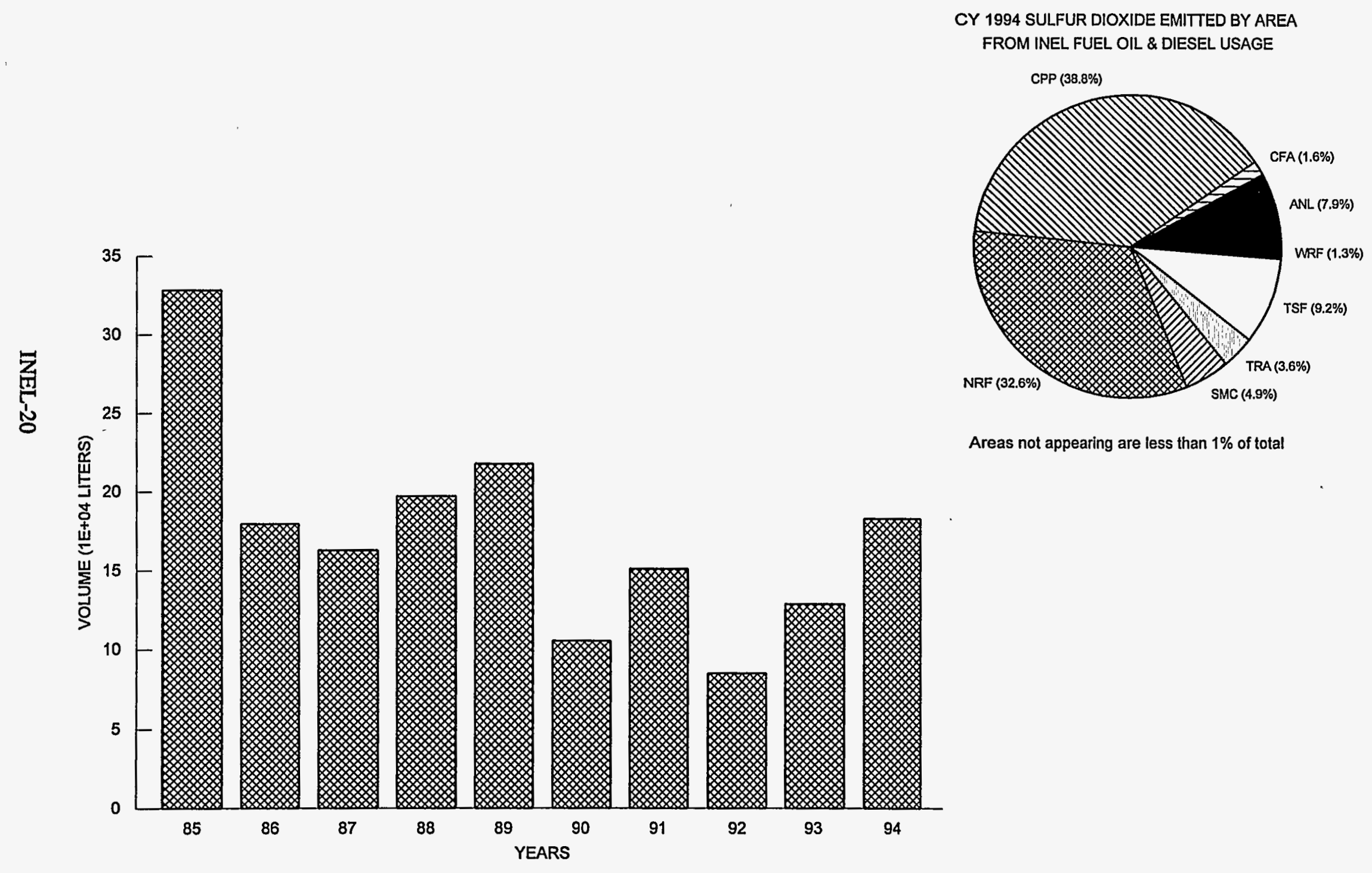




\section{CY 1985 - 1994 PARTICULATE EMITTED FROM INEL FUEL OIL \& DIESEL USAGE}

CY 1994 PARTICULATE EMITTED BY AREA FROM INEL FUEL OIL \& DIESEL USAGE

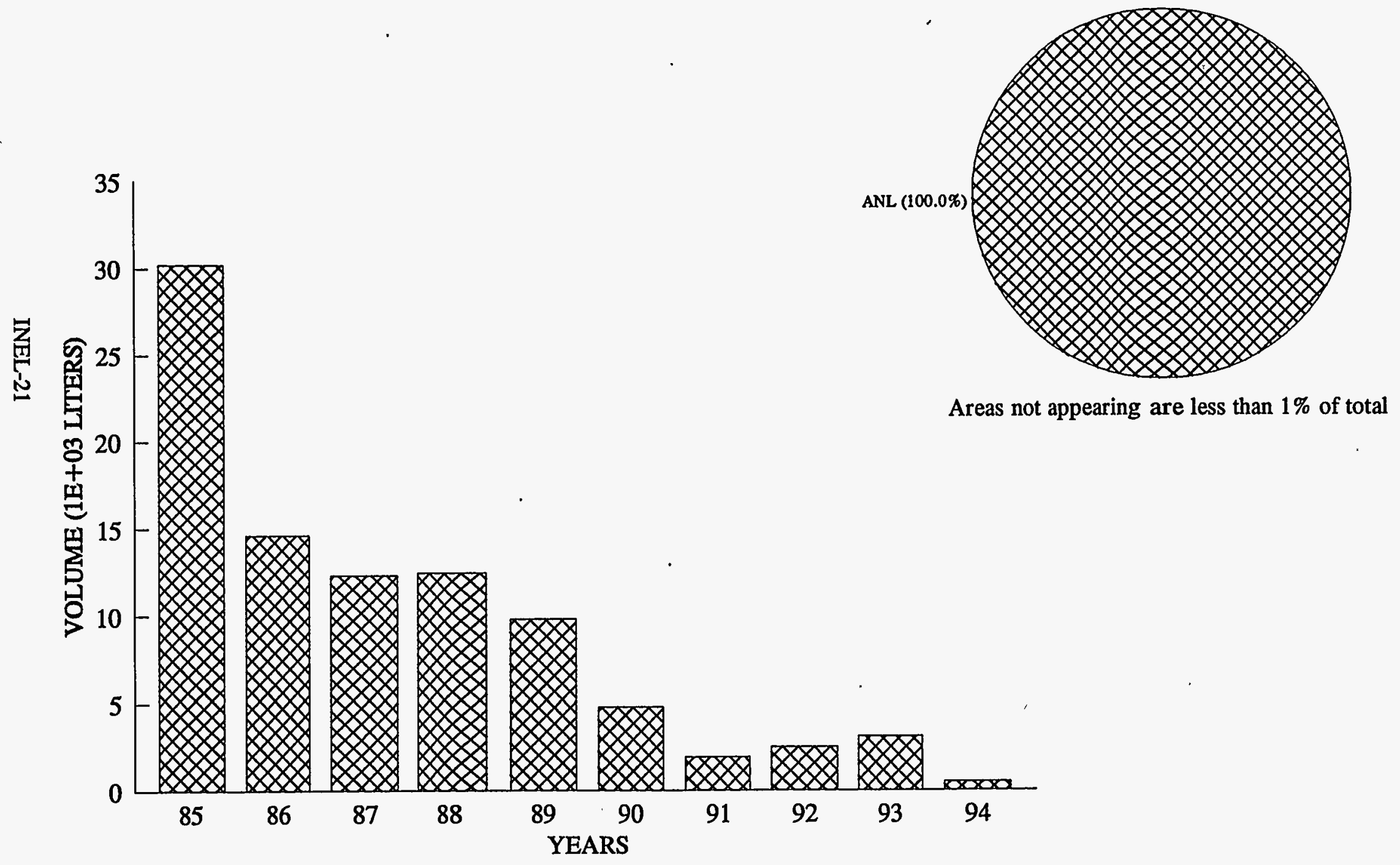


SCHED. NO INRPTO32A

INEL HATER USAGE SUMMARY IN THOUSAND LITERS

RECORD TO DATE

\begin{tabular}{|c|c|c|c|c|c|}
\hline AREA & 1971 - 1991 & 1992 & 1993 & 1994 & TOTAL \\
\hline ANL & $9,228,102$ & 388,768 & 387,283 & 278,651 & $10,282,805$ \\
\hline ARA & $1,451,289$ & & & & $1,451,289$ \\
\hline CFA & $5,180,759$ & 377,274 & 313,328 & 284,401 & $6,155,762$ \\
\hline CPP & $36,847,534$ & $2,502,782$ & $2,827,841$ & $2,071,447$ & $44,249,604$ \\
\hline CTF & 791,207 & 2,953 & & & 794,160 \\
\hline LOF & $2,655,483$ & & & & $2,655,483$ \\
\hline MHD & 359,802 & 700 & & & 360,502 \\
\hline NRF & $28,445,330$ & $1,112,774$ & 884,395 & 592,432 & $31,034,931$ \\
\hline PBF & 673,636 & 15,227 & 11,452 & 16,251 & 716,566 \\
\hline TAN & $3,581,392$ & & & & $3,581,392$ \\
\hline TRA & $60,972,940$ & $2,255,909$ & $1,971,516$ & $1,764,286$ & $66,964,651$ \\
\hline TSF & 574,278 & 148,431 & 88,024 & 101,861 & 912,594 \\
\hline WMC & 389,483 & 1,814 & 3,511 & 1,767 & 396,576 \\
\hline WRF & 618,098 & 10,455 & 10,596 & 8,620 & 647,769 \\
\hline TOTAL & $151,769,335$ & $6,817,087$ & $6,497,948$ & $5,119,716$ & $170,204,084$ \\
\hline
\end{tabular}



RECORD TO DATE

\begin{tabular}{|c|c|c|c|c|c|}
\hline AREA & $1971-1991$ & 1992 & 1993 & 1994 & TOTAL \\
\hline ANL & $10,743,126$ & 405,378 & 475,408 & 372,922 & $11,996,836$ \\
\hline ARA & $1,961,500$ & & & & $1,961,500$ \\
\hline CFA & $8,956,192$ & 521,041 & 633,451 & 614,290 & $10,724,974$ \\
\hline CPP & $40,794,702$ & $3,069,581$ & $2,708,351$ & $2,076,670$ & $48,569,305$ \\
\hline CTF & $1,004,599$ & 2,965 & & & $1,007,564$ \\
\hline LOF & $3,326,689$ & & & & $3,326,689$ \\
\hline MHD & 369,829 & 700 & & & 370,530 \\
\hline NRF & $34,349,157$ & $1,110,924$ & 883,217 & 592,449 & $36,935,747$ \\
\hline PBF & $1,013,296$ & 18,129 & 13,801 & 19,742 & $1,064,968$ \\
\hline TAN & $4,434,485$ & & & & $4,434,485$ \\
\hline TRA & $68,687,688$ & $2,224,786$ & $1,962,878$ & $1,764,693$ & $74,640,044$ \\
\hline TSF & 595,501 & 94,453 & 98,575 & 101,819 & 890,347 \\
\hline IMC & 466,556 & 157,161 & 19,411 & 36,537 & 679,664 \\
\hline HRF & 627,413 & 12,795 & 8,870 & 8,327 & 657,406 \\
\hline TOTAL & $177,250,734$ & $7,617,914$ & $6,803,963$ & $5,587,450$ & $197,260,060$ \\
\hline
\end{tabular}




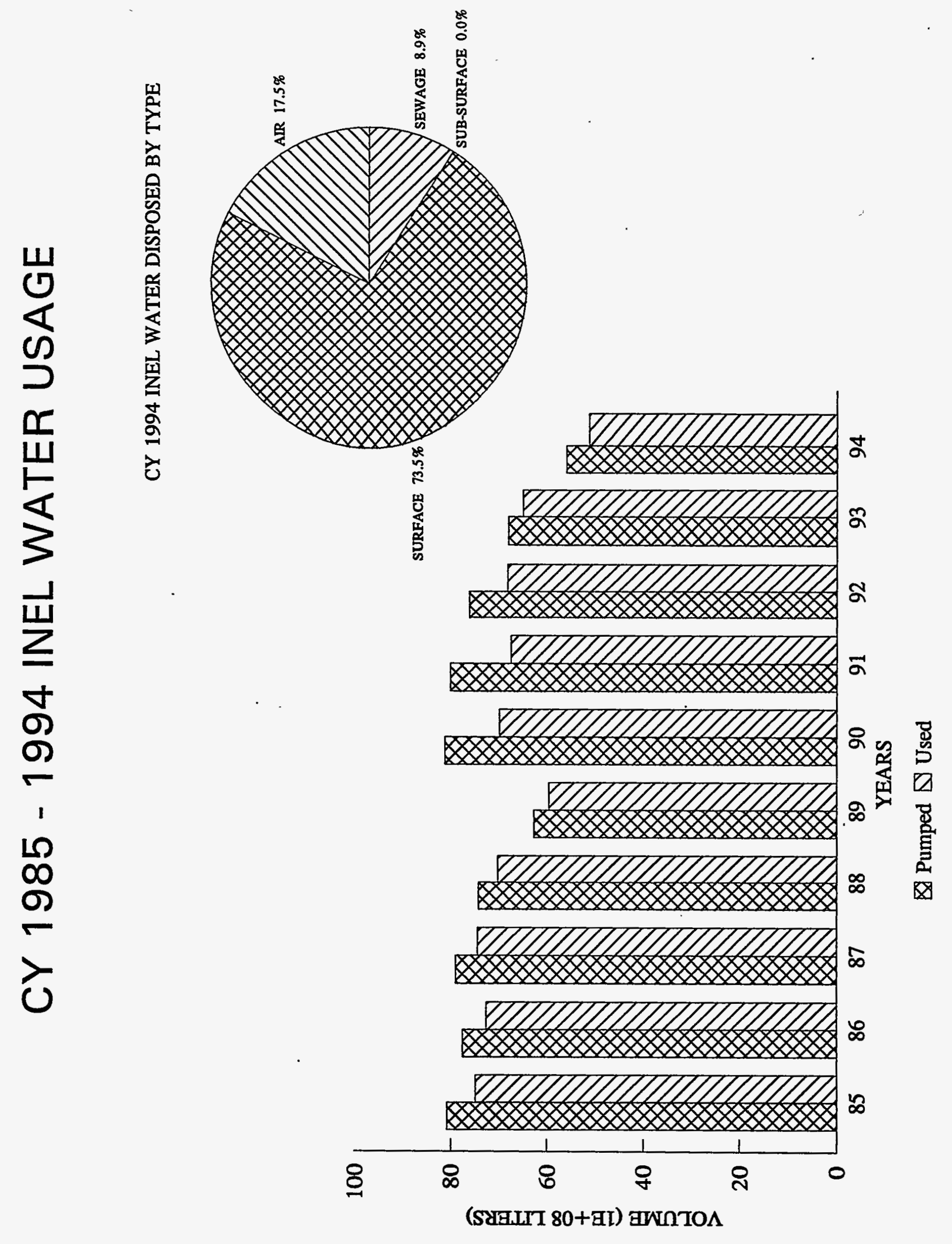

INEL-24 


\section{CY 1994 INEL WATER USAGE}

CY 1994 INEL WATER PUMPED BY AREA

㿣

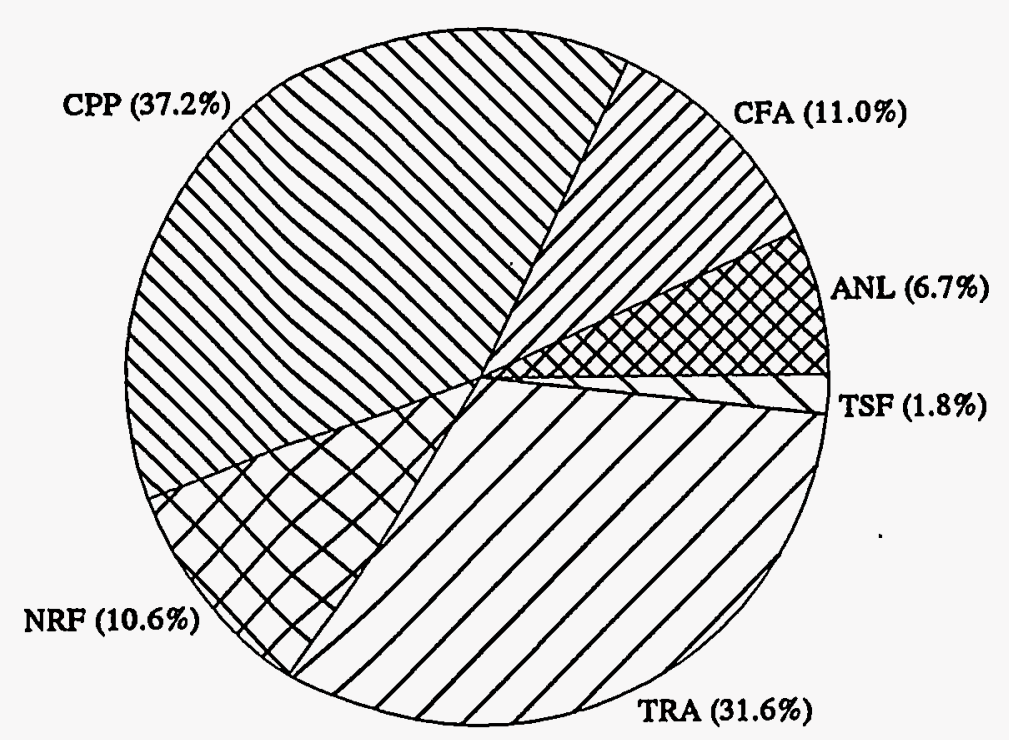

Areas not appearing are less than $1 \%$ of total
CY 1994 INEL WATER DISPOSED BY AREA

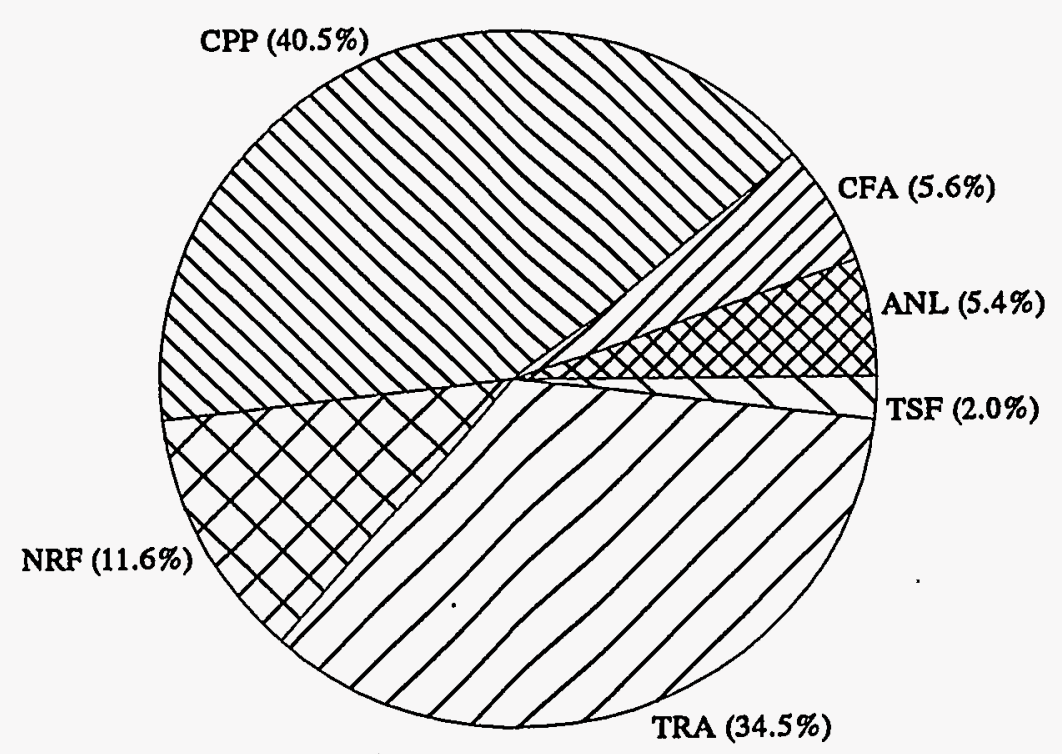

Areas not appearing are less than $1 \%$ of total 


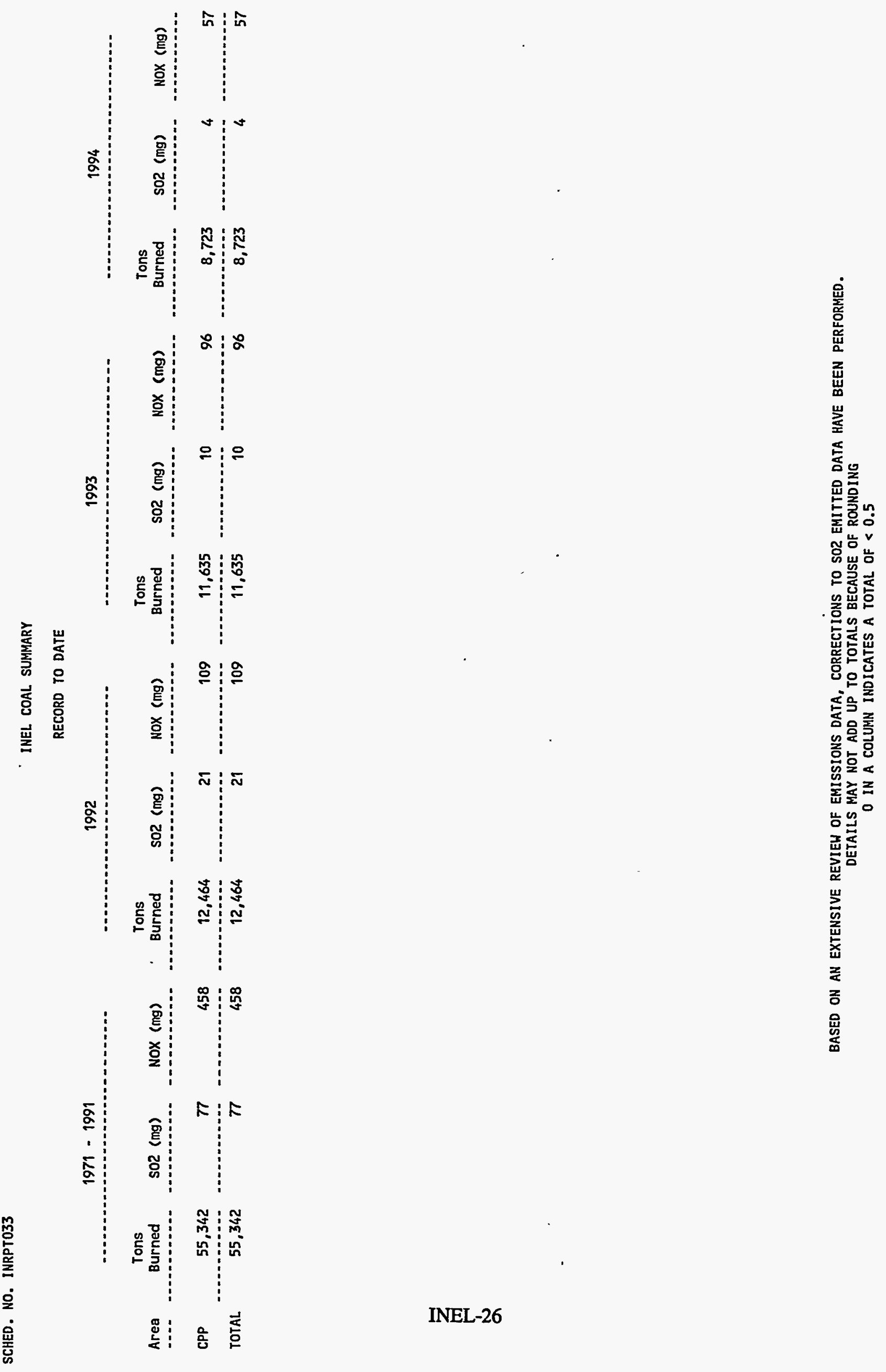


SCHED. NO. INRPT033

\begin{tabular}{|c|c|c|c|}
\hline \multirow[b]{2}{*}{ Area } & \multicolumn{3}{|c|}{ TOTALS } \\
\hline & $\begin{array}{c}\text { Tons } \\
\text { Burned }\end{array}$ & s02 (mg) & NOK (mg) \\
\hline CPP & 88,164 & 112 & 720 \\
\hline & 88,164 & 112 & 720 \\
\hline
\end{tabular}

INEL COAL SUMMARY

RECORD TO DATE (CONTINUED)

BASED ON AN EXTENSIVE REVIEH OF EMISSIONS DATA, CORRECTIONS TO SOZ EMITTED DATA HAVE BEEN PERFORMED. DETAILS MAY NOT ADD UP TO TOTALS BECAUSE OF ROUNDING

0 IN A COLUMN INDICATES A TOTAL OF $<0.5$ 


\section{CY 1987 - 1994 INEL COAL USAGE}

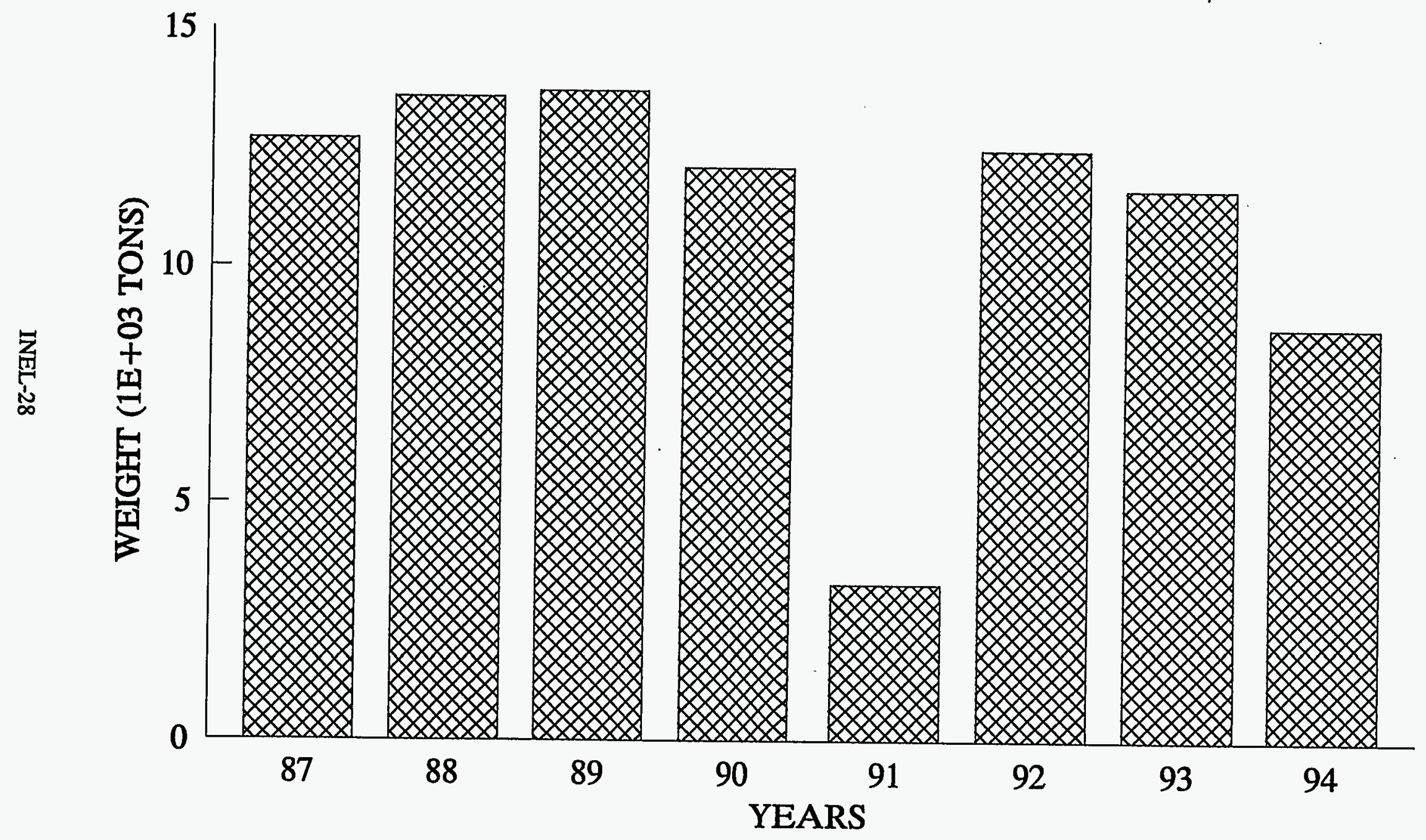




\section{CY 1987 - 1994 SULFUR DIOXIDE EMITTED FROM INEL COAL USAGE}

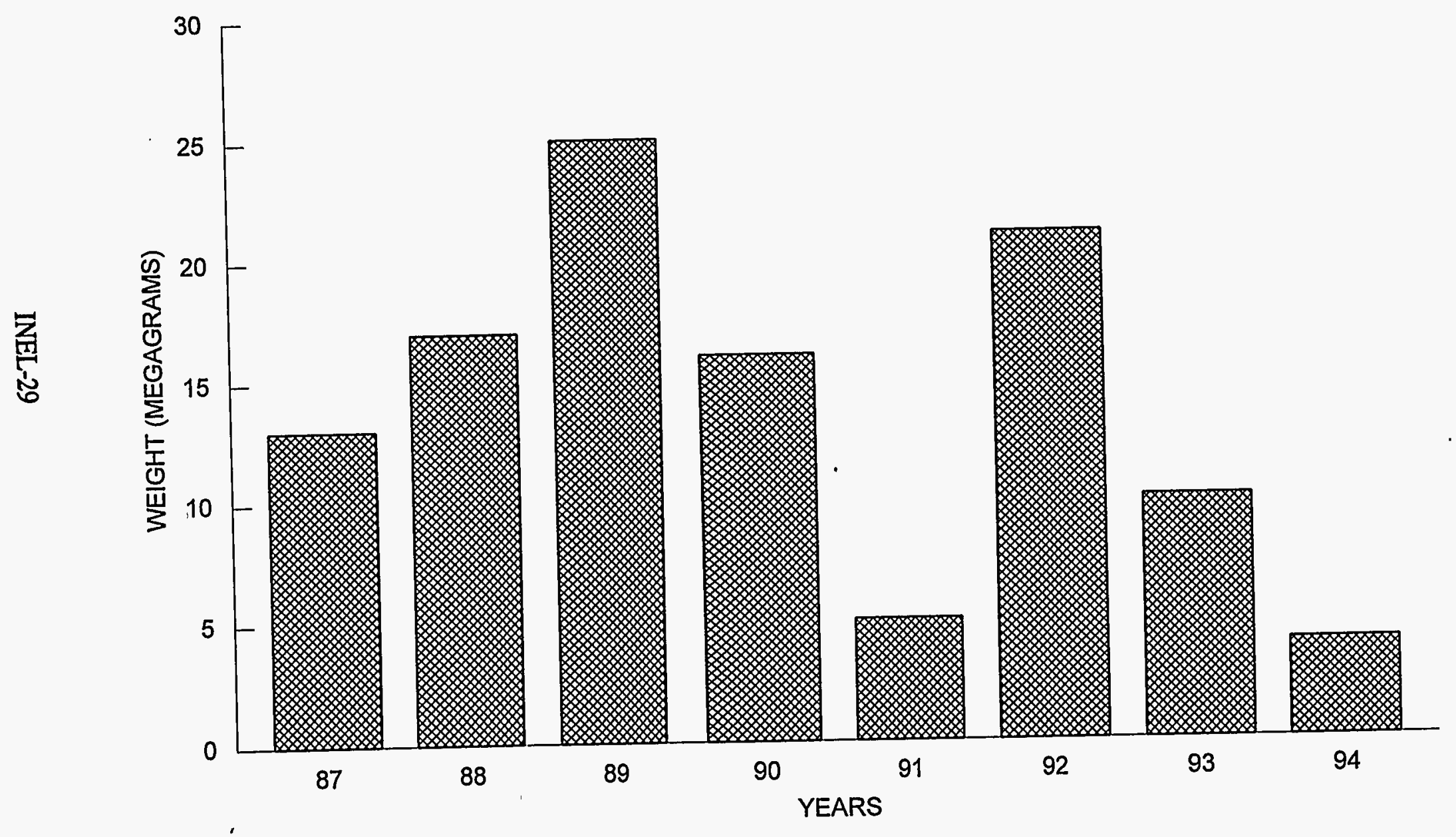



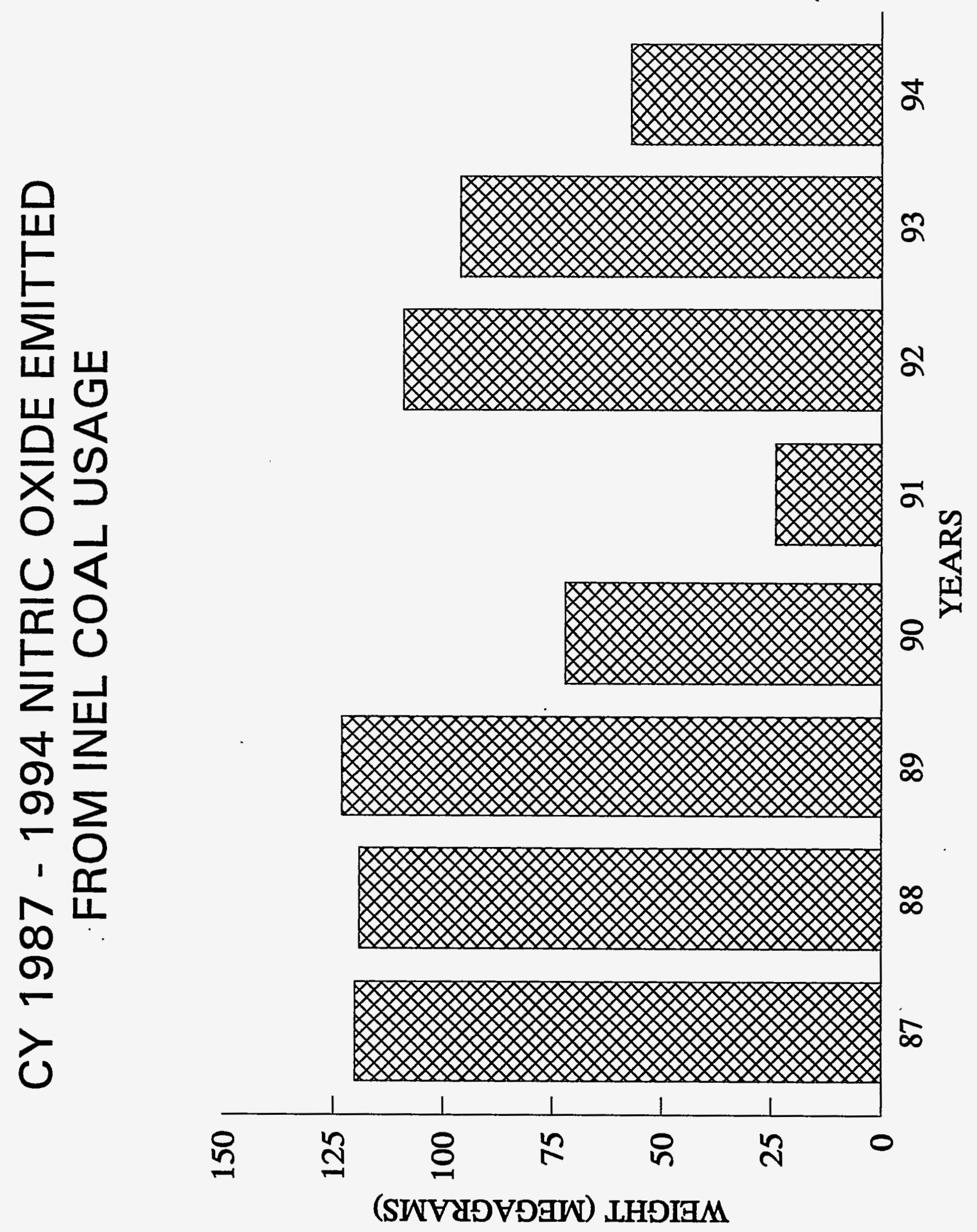
SCHED. NO. INRPT034

IHEL HASTE OIL SUMMARY IN LITERS

RECORD TO DATE

\begin{tabular}{|c|c|c|c|c|c|c|c|c|c|c|c|c|}
\hline AREA & $1971-1984$ & 1985 & 1986 & 1987 & 1988 & 1989 & 1990 & 1991 & 1992 & 1993 & 1994 & TOTAL \\
\hline ANL & 27,741 & 2,425 & 1,230 & 1,052 & 3,218 & 2,173 & & & 2,271 & 568 & & 40,679 \\
\hline ARA & & & & 208 & 341 & & & 1,344 & & & & 1,893 \\
\hline BRX & & & & & & & & 454 & & & & 454 \\
\hline CFA & & & 27,028 & 40,598 & 74,818 & 35,412 & 30,586 & 50,660 & 25,165 & 21,444 & & 305,713 \\
\hline CPP & & & 3,785 & 3,785 & & & 6,871 & & 3,028 & & & 17,470 \\
\hline IRC & & & & 416 & & & & 927 & 189 & 189 & & 1,722 \\
\hline NRF & 418,260 & 10,115 & 50,403 & 70,976 & 77,650 & 129,499 & 38,157 & 25,268 & 11,545 & 22,042 & 12,011 & 865,925 \\
\hline PBF & 4,997 & & & 492 & 416 & 208 & & 17,091 & 189 & & & 23,394 \\
\hline PTI & & & & & & 208 & & 1,136 & & & & 1,344 \\
\hline TAN & & & 3,123 & 5,072 & 13,325 & 1,420 & 17,413 & 14,195 & 757 & 757 & & 56,062 \\
\hline TRA & 5,678 & & 4,921 & 7,238 & 3,312 & 12,870 & 16,448 & 6,057 & 10,221 & 5,678 & & 72,422 \\
\hline WER & & & & & & & & & & 852 & & 852 \\
\hline WMC & & & 757 & 1,666 & 833 & 1,136 & 625 & 208 & . & & . & 5,224 \\
\hline HRF & & & & 208 & 1,079 & 341 & 1,325 & 4,164 & & & & 7,117 \\
\hline & 456,676 & 12,540 & 91,247 & 131,713 & 174,991 & 183,266 & 111,423 & 121,504 & 53,367 & 51,531 & 12,011 & $1,400,269$ \\
\hline
\end{tabular}




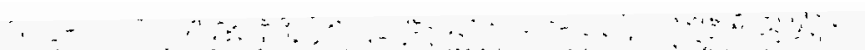




\section{INEL 1994 Year-to-Date Summaries}

INEL Landfill Disposed Solid Waste Summary In Cubic Meters

For January Through December, $1994 \ldots \ldots \ldots \ldots \ldots \ldots \ldots \ldots$. . . . . . INEL-35 INEL Landfill Disposed Solid Waste Summary, Fourth Quarter and

Year-To-Date $1994 \ldots \ldots \ldots \ldots \ldots \ldots \ldots \ldots \ldots \ldots \ldots \ldots$. . . . . . . . . . . . . . . .

Airborne And Liquid Industrial Waste Substances Summary

In Kilograms For January Through December, $1994 \ldots \ldots \ldots \ldots \ldots$. . . . INEL-37

INEL Year-To-Date Water Usage Summary For

January Through December $1994 \ldots \ldots \ldots \ldots \ldots \ldots \ldots \ldots \ldots$ INEL-39

INEL Quarterly Fuel Oil And Diesel Summary For

January Through December $1994 \ldots \ldots \ldots \ldots \ldots \ldots \ldots \ldots$ INEL-40

INEL Waste Oil Summary In Gallons For January Through December 1994 . . . . INEL-41

INEL Year-To-Date Coal Usage Summary For

January Through December 1994 . . . . . . . . . . . . . . . . . . . INEL-42

Summary INEL Hazardous Waste Generated For January-December $1994 \ldots$. . . INEL-43

Summary INEL Mixed Waste Generated For January-December 1994 . . . . . . INEL-44 
SCHED. NO. INRPT038

INEL LANDFILL DISPOSED SOLID HASTE SUMMARY IN CUBIC METERS

FOR JANUARY THROUGH DECEMBER, 1994

RPT 3-I

\begin{tabular}{|c|c|c|c|c|c|c|c|c|c|c|}
\hline Type of Haste & ANL & CFA & CPP & DAD & NRF & PBF & TAN & TRA & WMC & TOTALS \\
\hline ASBESTOS & 31 & 147 & 203 & & 305 & & 64 & 29 & & 779 \\
\hline ASPHALT & 1,488 & 133 & 3,444 & & 1,099 & 12 & 48 & 1 & & 6,226 \\
\hline CAFETERIA GARBAGE & 668 & 1,965 & 31. & & 1,002 & & 9 & & & 3,675 \\
\hline OIRT AND GRAVEL & 398 & 1,114 & 23,273 & 0 & 100 & 11 & 34 & 2 & 155 & 25,087 \\
\hline MASONRY AND CONCRETE & 248 & 2,733 & 709 & 14 & 214 & 6 & 417 & 20 & 2 & 4,364 \\
\hline SCRAP METAL & 1,041 & 404 & 380 & 53 & 185 & 27 & 254 & 154 & 21 & 2,518 \\
\hline TRASH， SWEEPINGS, ETC. & 5,995 & 6,394 & 6,751 & 8 & 7,220 & 638 & 2,291 & 2,062 & 810 & 32,170 \\
\hline HEEDS, GRASS AND TREES & 15 & 53 & 16 & & 8 & 3 & & 1 & 2 & 98 \\
\hline WOOD AND SCRAP LUMBER & 629 & 377 & 588 & 19 & 186 & 26 & 88 & 152 & 55 & 2,119 \\
\hline TOTALS & 10,512 & 13,320 & 35,395 & 95 & 10,319 & 722 & 3,206 & 2,421 & 1,045 & 77,036 \\
\hline
\end{tabular}


PAGE: 1

SCHED. NO. INRPTOO3
IDAHO OPERATIONS OFFICE

UNITED STATES DEPARTMENT OF ENERGY

INEL NONRADIOLOGICAL WASTE MANAGEMENT INFORMATION SYSTEM

INEL LANDFILL DISPOSED SOLID WASTE SUMMARY

FOURTH QUARTER AND YEAR-TO-DATE 1994
$10-J U L-95$

PRT 3-1

\begin{tabular}{|c|c|c|c|c|c|c|c|c|c|c|c|c|c|}
\hline AREA & JAN & FEB & MAR & APR & MAY & $\begin{array}{l}\text { WASTE VOL } \\
\text { JUN }\end{array}$ & $\begin{array}{r}\text { ME (CH) } \\
\text { JUL }\end{array}$ & AUG & SEP & OCT & NOV & DEC & TOTAL \\
\hline ANL & 675.5 & 599.4 & 618.6 & 624.7 & 561.2 & 681.3 & 729.4 & 922.1 & $1,862.2$ & $1,866.0$ & 945.0 & 426.6 & $10,512.1$ \\
\hline CFA & 803.6 & 724.8 & $1,022.7$ & 686.2 & $1,412.2$ & 983.3 & $1,283.4$ & $1,083.4$ & $1,520.0$ & $1,536.1$ & $1,168.3$ & $1,096.1$ & $13,320.1$ \\
\hline CPP & 757.7 & 769.2 & $6,335.5$ & $6,454.4$ & $2,495.3$ & $1,926.4$ & $4,081.1$ & $4,467.2$ & $2,671.9$ & $3,238.5$ & $2,009.8$ & 188.5 & $35,395.2$ \\
\hline DAD & & & & & & & & & & & & 94.8 & 94.8 \\
\hline NRF & 838.8 & 695.0 & 850.2 & 926.7 & 831.1 & 757.0 & 728.7 & $1,212.7$ & $1,135.0$ & $1,015.0$ & 857.5 & 471.8 & $10,319.4$ \\
\hline PBF & 91.0 & 45.5 & 87.9 & 68.0 & 63.8 & 36.7 & 38.6 & 83.0 & $40: 9$ & 73.0 & 55.1 & 38.6 & 722.2 \\
\hline TAN & 215.6 & 217.9 & 189.6 & 230.9 & 280.6 & 285.2 & 172.0 & 298.2 & 656.8 & 257.7 & 251.6 & 149.9 & $3,206.0$ \\
\hline TRA & 196.1 & 239.7 & 310.0 & 310.8 & 182.7 & 355.2 & 135.3 & 214.5 & 133.4 & 122.7 & 134.6 & 85.6 & $2,420.7$ \\
\hline WMC & 61.6 & 61.2 & 86.0 & 71.9 & 57.7 & 78.8 & 84.1 & 89.1 & 110.5 & 215.2 & 57.3 & 71.9 & $1,045.2$ \\
\hline TOTAL & $3,639.9$ & $3,352.8$ & $9,500.5$ & $9,373.6$ & $5,884.7$ & $5,103.7$ & $7,252.6$ & $8,370.1$ & $8,130.8$ & $8,324.2$ & $5,479.1$ & $2,623.7$ & $77,035.7$ \\
\hline
\end{tabular}


FOR JANUARY THROUGH DECEMBER, 1994

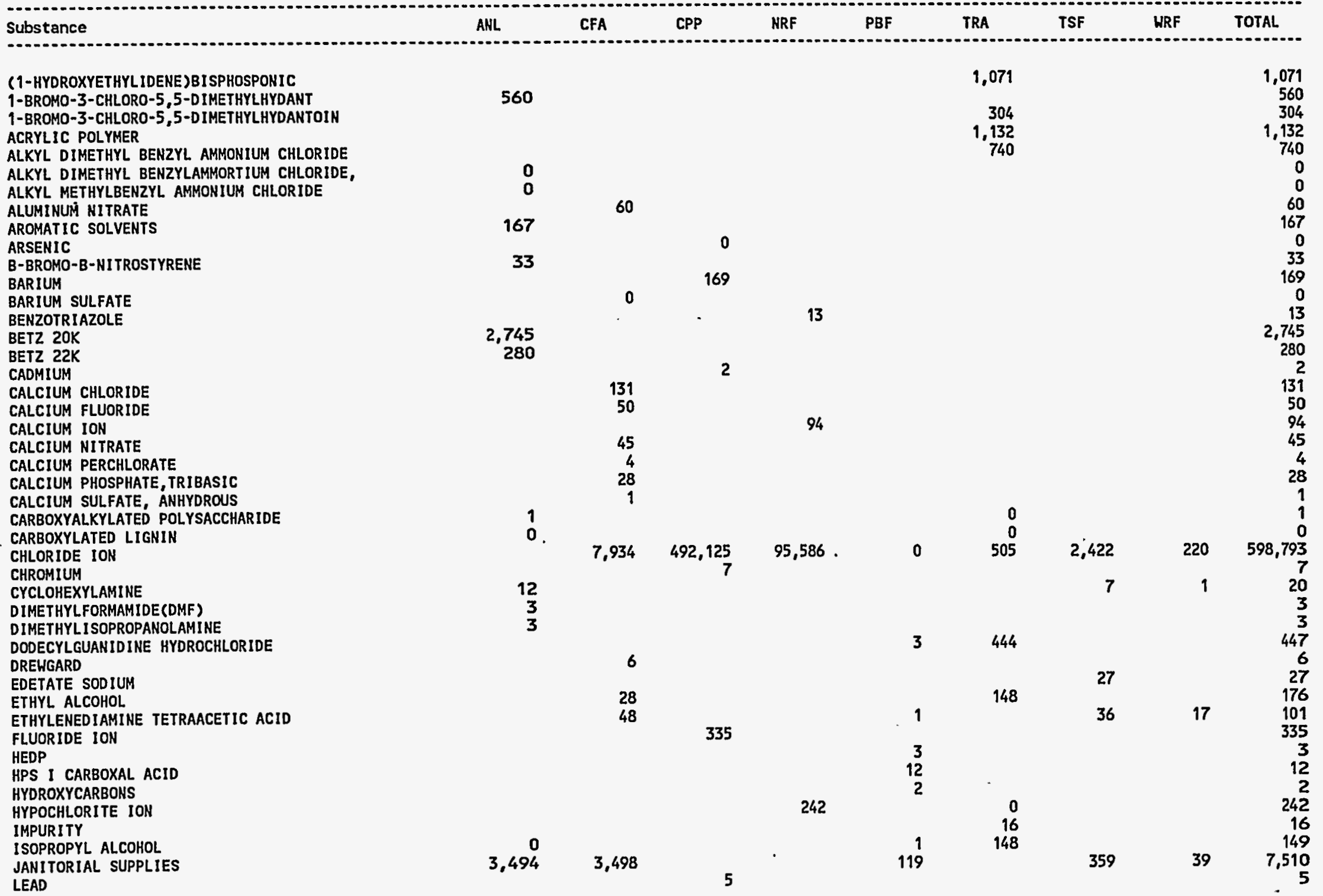

DETAIL MAY NOT ADD UP TO TOTALS BECAUSE OF ROUNDING 0 IN A COLUMH INDICATES A TOTAL OF $<0.5$ 
FOR JANUARY THROUGH DECEMBER, 1994

Substance

LIGNOSULFONIC ACID, SODIUM SALT

METHYLENE BIS(THIOCYANATE)

MOLYBDATE SALT

MORPHOLINE

$N$-HYDROCARBON

$N$-METHYLPYRROLIDONE

NITRATE ION

NITRIC OXIDE

NITRITE SALT

NITROGEN DIOXIDE

MONYLPHENOXYPOLY(ETHYLENEOXY)ETHANO

NONYLPHENOXYPOLY(ETHYLENEOXY) ETHANOL

ORTHO PHOSPHATE

PHOSPHATE

PHOSPHATE ION

POLYACRYLATE

POLYACRYLATE ACID

POLYALYYLEE ACID

POTASSIUM HYDROXIDE

POTASSIUM ION

PYRO PKOSPHATE

SALT OF EDTA

SILICONE EMULSION

SILVER

SODIUM HYDRATE SOLUTION

SODIUM HYDROXIDE

SODIUM HYPOCHLORITE

SODIUM ION

SODIUM LIGNOSULFONATE

SODIUM SULFATE

SODIUM SULFITE

SODIUM TOLYTRIAZOLE

SODIUM TRIPOLYPHOSPHATE

STODDARD SOLVENT

SULFATE ION

SULFITE ION

TETRAPOTASSIUM PYROPHOSPHATE

TOLYTRIAZOLE

OTAL HARDNESS

TRIBUTYLTIN OXIDE

Total

ANL

CPP

NRF

PBF

TRA

TSF

TOTAL

15

47$$
\begin{array}{r}
12.198 \\
0
\end{array}
$$

0

13

o

48

0

\begin{tabular}{rrrr} 
& \multicolumn{3}{c}{4} \\
73 & & & 39 \\
3,970 & 5,288 & 293,099 & 45,049 \\
1 & & & \\
5 & & & \\
2 & & & \\
83 & & & \\
36,526 & 243 & 48,704 & 87,442
\end{tabular}

10

1
0
9

148

27

8

7

809

$\begin{array}{rr}3 & \\ 0 & \\ 13 & 1,924 \\ 2 & 1,071 \\ 1 & \\ 4 & \\ 0 & \end{array}$

924
$071 \quad 36$

73,937
.30

23,735

0
0
1,071
0

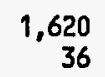

36

149

27
164
0
8
9
47

12,198

0

0

17

.174

0

181,832

285,661
2,677

2,677

184,500

148

762,081

4,664

$4751,908,093$

DETAIL MAY NOT ADD UP TO TOTALS BECAUSE OF ROUNDING

$O$ IN A COLUMN INDICATES A TOTAL OF $<0.5$ 
PAGE: 1

SCHED. NO. INRPT002-A
IOAHO OPERATIONS OFFICE

10-JUL-95

INEL NONRADIOLOGICAL. WASTE MANAGEMENT INFORMATION SYSTEM

INEL YEAR-TO-DATE WATER USAGE SUMMARY

FOR JANUARY THROUGH DECEMBER 1994

RPT 2-I

(ALL VOLUMES = NEAREST THOUSAND LITERS)

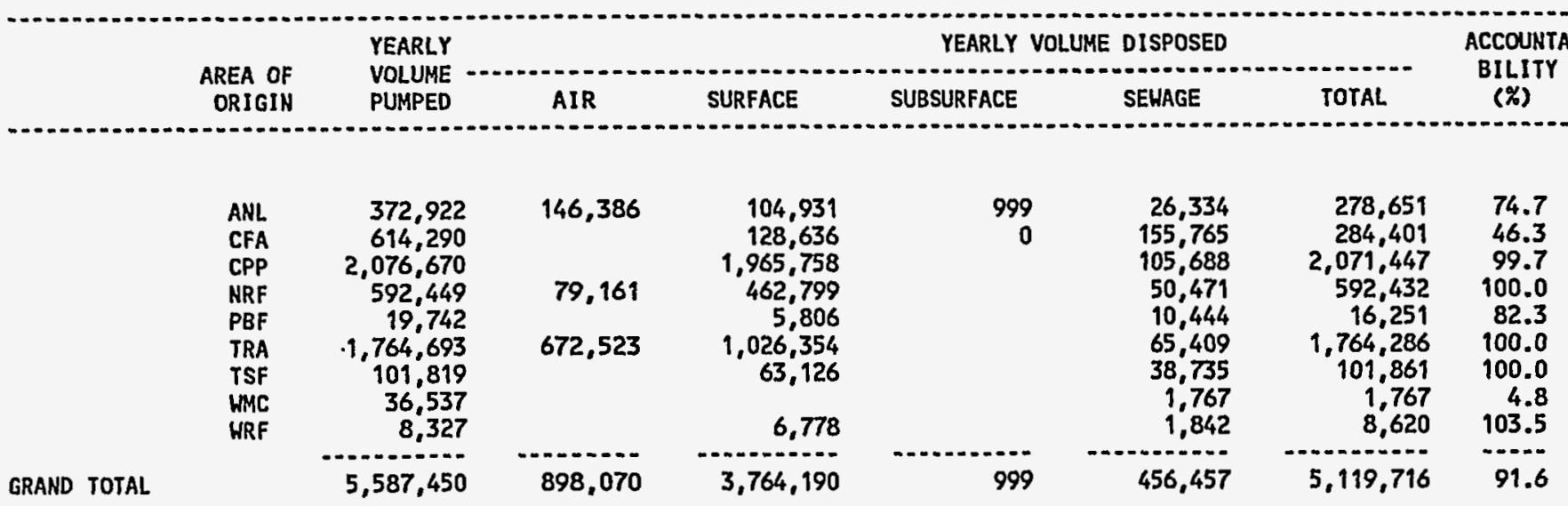

DETAIL MAY NOT ADD UP TO TOTALS BECAUSE OF ROUNDING

$O$ IH A COLUMN INDICATES A TOTAL OF $<0.5$ 
PAGE: 1

SCHED. NO. INRPTOO6
IDAHO OPERATIONS OFFICE

10-JUL-95

UNITED STATES DEPARTMENT OF ENERGY

INEL NONRADIOLOGICAL. WASTE MANAGEMENT INFORMATION SYSTEM

INEL QUARTERLY FUEL OIL AND DIESEL SUMMARY FOR JANUARY THROUGH DECEMBER 1994
RPT 5-1

FUEL OIL IN LITERS

KILOGRAMS DISCHARGED

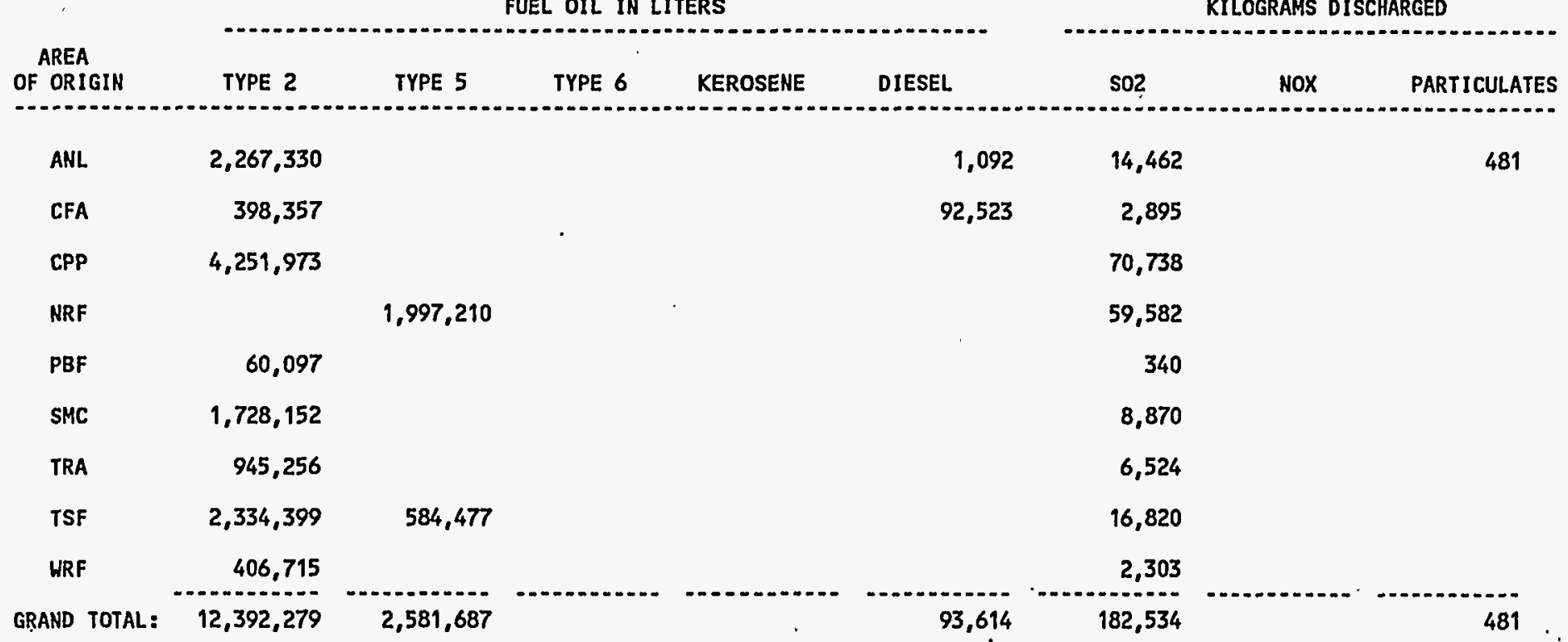


PAGE: 1

SCHED. NO. INRPTOI6
JDAHO OPERATIONS OFFICE

UHITED STATES DEPARTMENT OF ENERGY

INEL. NONRADIOLOGICAL HASTE MANAGENENT INFORMATION SYSTEN

INEL HASTE OIL SUMMARY IN GALLONS
RPT 9-1

JANUARY THROUGH DECEMBER 1994

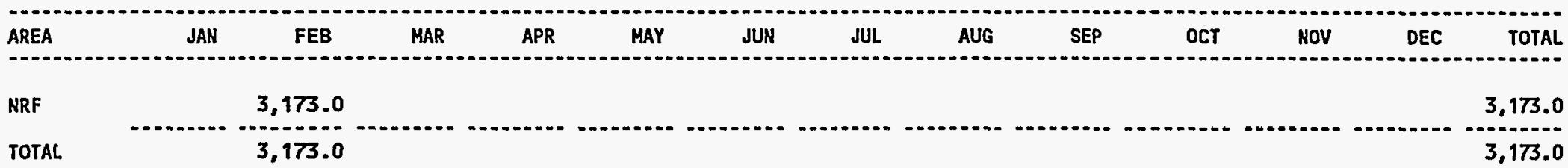


PAGE: 1

SCHED. NO. INRPT018
IDAHO OPERATIONS OFFICE

$10-J U L-95$

UNITED STATES DEPARTMENT OF ENERGY

INEL NONRADIOLOGICAL WASTE MANAGEMENT INFORMATION SYSTEM

INEL YEAR-TO-DATE COAL USAGE SUMMARY

FOR JANUARY THROUGH DECEMBER 1994
RPT 5-I

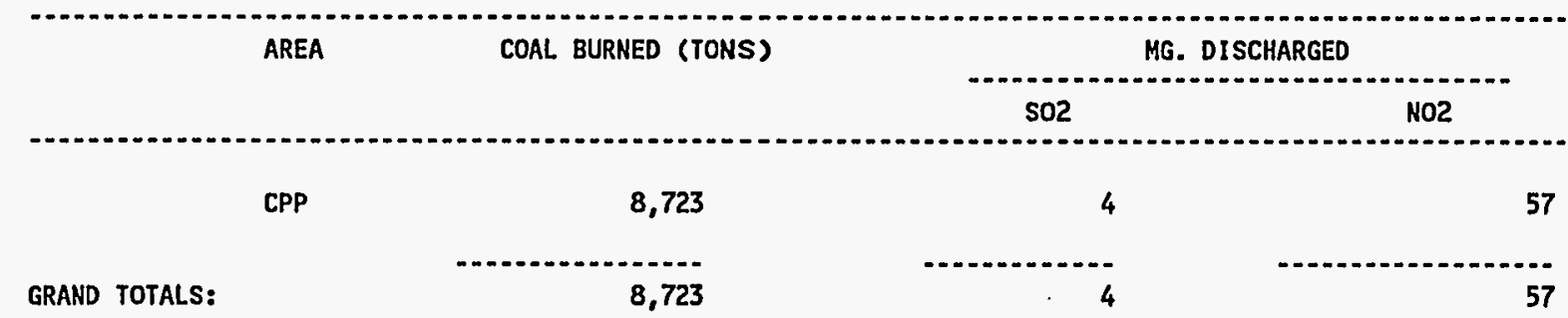

BASED ON AN EXTENSIVE REVIEH OF EMISSIONS DATA, CORRECTIONS TO SO2 EMITTED DATA HAVE BEEN PERFORMED. DETAILS MAY NOT ADD UP TO TOTALS BECAUSE OF ROUNDING

0 IN A COLUMN INDICATES A TOTAL OF $<0.5$ 
IDAHO OPERATIONS OFFICE

UNITED STATES DEPARTMENT OF ENERGY

INEL NONRADIOLOGICAL WASTE MANAGEMENT INFORMATION SYSTEM

SUMMARY INEL

HAZARDOUS WASTE GENERATED

FOR January - December 1994

RPT 10-I

\begin{tabular}{|c|c|c|c|c|c|c|c|c|c|c|c|c|c|}
\hline AREA & JAN & FEB & MAR & APR & MAX & JUN & JUL & AUG & SEP & OCT & Nov & DEC & YTD \\
\hline ANL & $1.135 E+03$ & & & $5.250 \mathrm{E}+02$ & $8.450 E+02$ & & $2.023 E+03$ & $7.270 \mathrm{E}+02$ & $1.606 \mathrm{E}+03$ & & $9.320 \mathrm{E}+02$ & $1.714 E+03$ & $9.507 E+03$ \\
\hline ARA & & & & & & & $1.150 \mathrm{E}+02$ & & & & $2.040 E+03$ & & $2.155 E+-3$ \\
\hline CFA & $2.625 \mathrm{E}+03$ & & $6.260 \mathrm{E}+02$ & $6.040 \mathrm{E}+02$ & $1.488 \mathrm{E}+03$ & & $5.410 \mathrm{E}+02$ & $7.900 \mathrm{E}+02$ & $2.810 \mathrm{E}+03$ & $6.790 \mathrm{E}+02$ & & $3.600 \mathrm{E}+01$ & $1.020 \mathrm{E}+04$ \\
\hline CPP & & & & $1.300 \mathrm{E}+02$ & $2.500 \mathrm{E}+01$ & $1.581 E+04$ & & & $5.790 \mathrm{E}+02$ & & & $3.816 E+03$ & $2.036 \mathrm{E}+04$ \\
\hline IRC & $7.100 E+01$ & & $8.600 \mathrm{E}+02$ & $2.280 \mathrm{E}+02$ & $1.786 E+03$ & & & $1.452 \mathrm{E}+03$ & & $1.930 \mathrm{E}+02$ & & $2.325 E+03$ & $6.915 E+03$ \\
\hline NRF & $1.486 \mathrm{E}+03$ & & $9.717 E+03$ & $5.381 \mathrm{E}+03$ & $6.790 \mathrm{E}+02$ & $2.692 \mathrm{E}+03$ & $1.190 \mathrm{E}+02$ & $2.697 E+04$ & $9.860 \mathrm{E}+02$ & $3.440 E+02$ & $8.600 \mathrm{E}+02$ & $8.230 \mathrm{E}+02$ & $5.006 \mathrm{E}+04$ \\
\hline PTI & & $4.000 \mathrm{E}+01$ & $1.600 \mathrm{E}+03$ & & & & & $2.070 \mathrm{E}+02$ & $9.600 \mathrm{E}+01$ & & & $1.070 \mathrm{E}+02$ & $2.050 \mathrm{E}+03$ \\
\hline TAN & $6.150 \mathrm{E}+02$ & & $1.200 \mathrm{E}+01$ & & & $3.630 E+02$ & $2.950 \mathrm{E}+02$ & $1.428 \mathrm{E}+05$ & $1.166 \mathrm{E}+05$ & & $1.117 E+05$ & $1.782 \mathrm{E}+04$ & $3.903 E+05$ \\
\hline TRA & $1.567 E+03$ & & & $4.730 E+02$ & $2.526 E+03$ & $2.090 \mathrm{E}+02$ & & & $6.210 \mathrm{E}+02$ & $5.060 \mathrm{E}+02$ & $1.100 E+01$ & & $5.913 E+03$ \\
\hline WCB & & & & & $1.130 \mathrm{E}+02$ & & & & & & & $6.200 \mathrm{E}+01$ & $1.750 \mathrm{E}+02$ \\
\hline WER & & & & $1.900 \mathrm{E}+01$ & & $2.000 \mathrm{E}+02$ & & & & & & . & $2.190 \mathrm{E}+02$ \\
\hline WMC & & & $1.609 \mathrm{E}+04$ & & & & & & & & & $5.750 \mathrm{E}+02$ & $1.666 \mathrm{E}+04$ \\
\hline $\begin{array}{l}\text { TOTAL } \\
\text { WEIGHT }\end{array}$ & $7.499 \mathrm{E}+03$ & $4.000 \mathrm{E}+01$ & $2.890 \mathrm{E}+04$ & $7.360 \mathrm{E}+03$ & $7.462 \mathrm{E}+03$ & $1.927 \mathrm{E}+04$ & $3.093 \mathrm{E}+03$ & $1.730 \mathrm{E}+05$ & $1.233 E+05$ & $1.722 \mathrm{E}+03$ & $1.156 \mathrm{E}+05$ & $2.727 \mathrm{E}+04$ & $5.145 E+05$ \\
\hline
\end{tabular}


IDAHO OPERATIONS OFFICE

UNITED STATES DEPARTMENT OF ENERGY

INEL NONRADIOLOGICAL WASTE MANAGEMENT INFORMATION SYSTEM

SUMMARY INEL

MEXED WASTE GENERATED

RPT 10-I

FOR January - December 1994

\begin{tabular}{|c|c|c|c|c|c|c|c|c|c|c|c|c|c|}
\hline AREA & JAN & FEB & MAR & APR & MAY & JUN & JUL & AUG & SEP & OCT & Nov & DEC & YTD \\
\hline ANL & & & & & & & & & & & & $3.500 \mathrm{E}+01$ & $3.500 \mathrm{E}+01$ \\
\hline ARA & $1.600 \mathrm{E}+04$ & & & & & & & & & & & & $1.600 E+04$ \\
\hline CFA & $3.510 \mathrm{E}+02$ & & & $9.500 E+01$ & & & & & & $4.300 \mathrm{E}+01$ & $9.900 \mathrm{E}+01$ & $2.300 \mathrm{E}+01$ & $6.110 \mathrm{E}+02$ \\
\hline CPP & & & & & & & & & & & $2.020 \mathrm{E}+02$ & & $2.020 \mathrm{E}+02$ \\
\hline IRC & & & & & & & & $4.800 E+01$ & & & & & $4.800 \mathrm{E}+01$ \\
\hline NRF & & & & & & $2.000 \mathrm{E}+01$ & & & & $2.535 E+03$ & & $5.260 E+02$ & $3.081 \mathrm{E}+03$ \\
\hline PBF & & & & & & $2.400 \mathrm{E}+01$ & & $\cdot$ & & & & & $2.400 \mathrm{E}+01$ \\
\hline TAN & & & & & $4.630 \mathrm{E}+03$ & $.3 .844 E+04$ & & & & & & $2.000 \mathrm{E}+02$ & $4.327 \mathrm{E}+04$ \\
\hline TRA & & & & $1.935 \mathrm{E}+03$ & : & $8.077 E+03$ & $3.400 \mathrm{E}+01$ & & $1.560 \mathrm{E}+02$ & $1.503 E+03$ & & $3.450 \mathrm{E}+03$ & $1.516 \mathrm{E}+04$ \\
\hline WER & $2.802 E+03$ & & & & & & & & & & & & $2.802 \mathrm{E}+03$ \\
\hline WMC & & & & & & & & & $2.550 \mathrm{E}+02$ & & & . & $2.550 \mathrm{E}+02$ \\
\hline $\begin{array}{c}\text { TOTAL } \\
\text { WEIGHT }\end{array}$ & $1.915 E+04$ & & & $2.030 \mathrm{E}+03$ & $4.630 \mathrm{E}+03$ & $4.656 \mathrm{E}+04$ & $3.400 \mathrm{E}+01$ & $4.800 \mathrm{E}+01$ & $4.110 \mathrm{E}+02$ & $4.081 E+03$ & $3.010 \mathrm{E}+02$ & $4.234 E+03$ & $8.148 E+04$ \\
\hline
\end{tabular}


Argonne National Laboratory-West 1994 Detail Data

Report 114-ANL Airborne Disposed Substances Summary . . . . . . . . . . ANL-3 Report 114-ANL Airborne Release Point And Disposed Substances Summary . ANL-5 Report 115-ANL Liquid Disposed Substances Summary . . . . . . . . . . . . . ANL-8 Report 115-ANL Release Point And Liquid Disposed Substances Summary . . . ANL-11 Report 116-ANL Landfill Solid Disposed Waste Summary . . . . . . . . . . . . ANL-15 Report 117-ANL Water Usage And Disposal Summary . . . . . . . . . . . . ANL-16 Report 118-ANL Fuel Oil Usage And Stack Effluents Summary . . . . . . . . . ANL-20 
-

. 


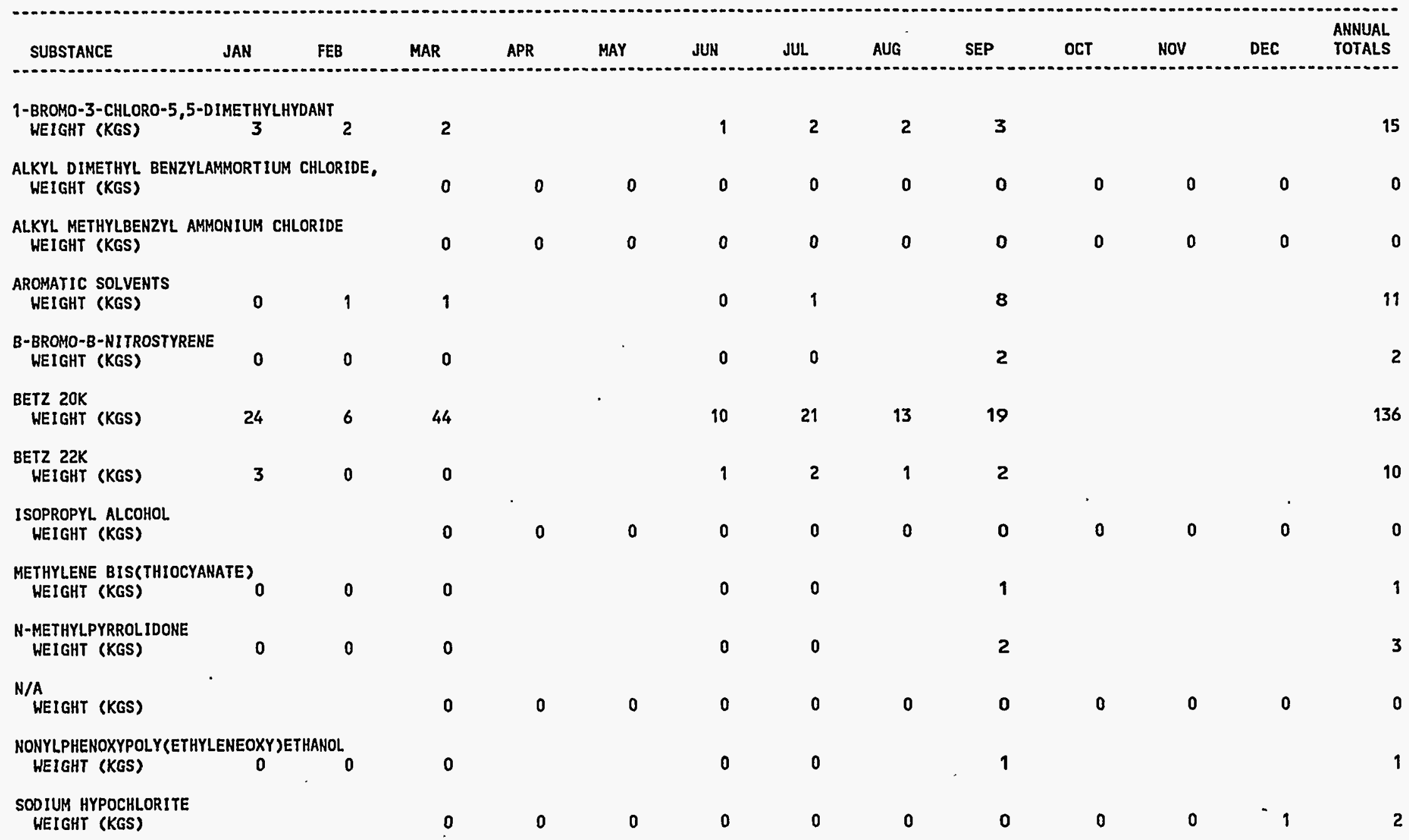


PAGE: 2

SCHED. NO. INRPTOOTA

IDAHO OPERATIONS OFFICE

11-JUL-95

UNITED STATES DEPARTMENT OF ENERGY

INEL NONRADIOLOGICAL WASTE MANAGEMENT INFORMATION SYSTEM

FOR JANUARY THROUGH DECEMBER 1994

ANL AIRBORNE DISPOSED SUBSTANCES SUMMARY

RPT 114-I

ANL

SUBSTANCE
$\begin{aligned} & \text { STODDARD SOLVENT } \\ & \text { WEIGHT }\end{aligned}$
(KGS)

$O$ IN A COLUMN INDICATES A TOTAL OF $<0.5$ 


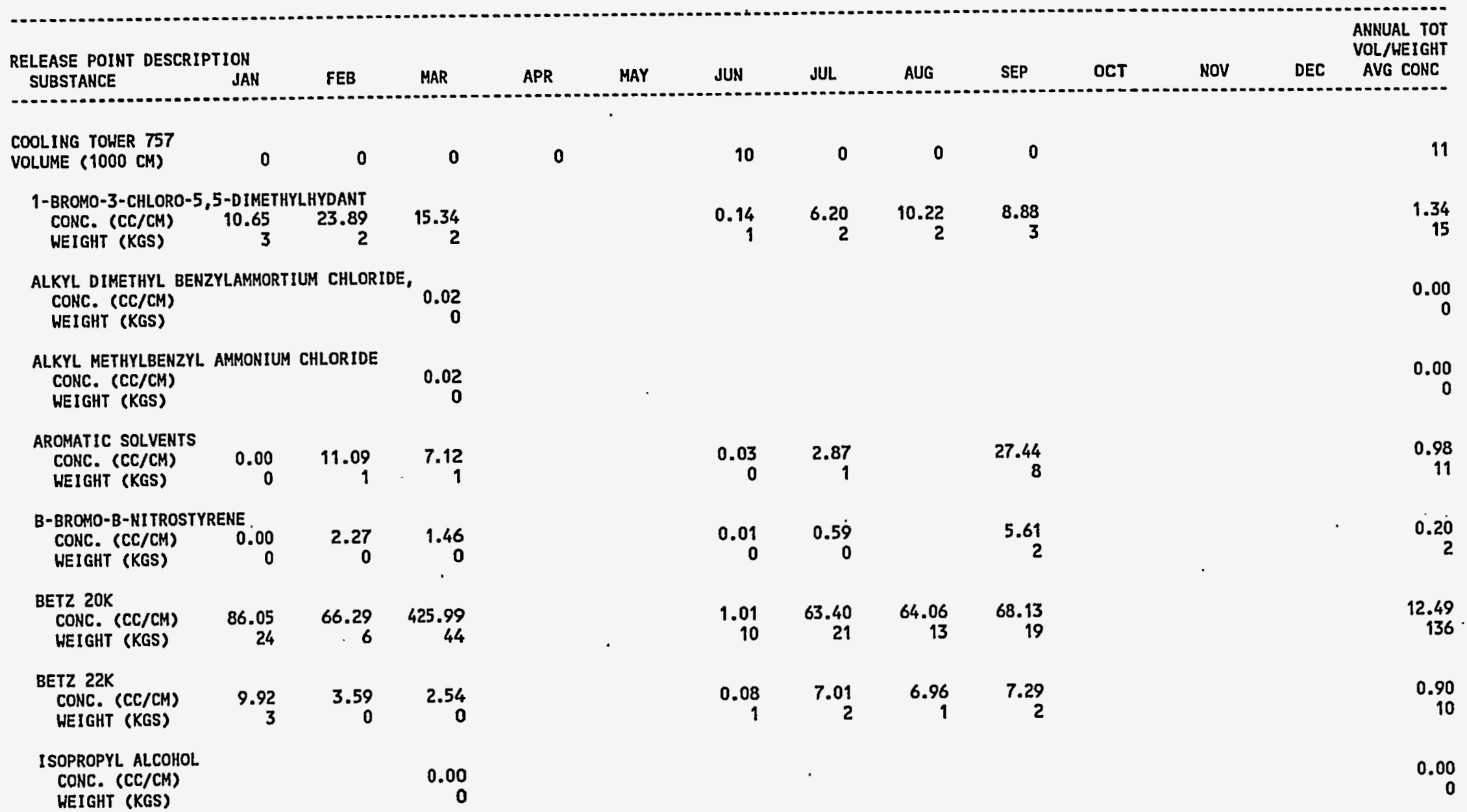




\begin{tabular}{|c|c|c|c|c|c|c|c|c|c|c|c|c|}
\hline $\begin{array}{l}\text { RELEASE POINT DESCRIPTION } \\
\text { SUBSTANCE }\end{array}$ & FEB & MAR & APR & MAY & JUN & JUL & AUG & SEP & OCT & NOV & DEC & $\begin{array}{l}\text { ANNUAL TOT } \\
\text { VOL/WEIGHT } \\
\text { AVG CONC }\end{array}$ \\
\hline \multicolumn{13}{|l|}{$\begin{array}{l}\text { COOLING TOWER } 757 \\
\text { METHYLENE BIS(THIOCYANATE) }\end{array}$} \\
\hline $\begin{array}{lr}\text { CONC. (CC/CM) } & 0.00 \\
\text { WEIGHT (KGS) } & 0\end{array}$ & $\begin{array}{r}1.01 \\
0\end{array}$ & $\begin{array}{r}0.65 \\
0\end{array}$ & & & $\begin{array}{r}0.00 \\
0\end{array}$ & $\begin{array}{r}0.26 \\
0\end{array}$ & & $\begin{array}{r}2.49 \\
1\end{array}$ & & & & $\begin{array}{r}0.09 \\
1\end{array}$ \\
\hline $\begin{array}{lr}\text { N-METHYLPYRROLIDONE } & \\
\text { CONC. (CC/CM) } & 0.00 \\
\text { WEIGHT (KGS) } & 0\end{array}$ & $\begin{array}{r}3.53 \\
0\end{array}$ & $\begin{array}{r}2.27 \\
0\end{array}$ & & & $\begin{array}{r}0.01 \\
0\end{array}$ & $\begin{array}{r}0.91 \\
0\end{array}$ & & $\begin{array}{r}8.73 \\
2\end{array}$ & & & & $\begin{array}{r}0.31 \\
3\end{array}$ \\
\hline $\begin{array}{l}\text { N/A } \\
\text { CONC. (CC/CM) } \\
\text { HEIGHT (KGS) }\end{array}$ & & $\begin{array}{r}0.00 \\
0\end{array}$ & & & & & & & & & & $\begin{array}{r}0.00 \\
0\end{array}$ \\
\hline $\begin{array}{cr}\text { NONYLPHENOXYPOLY (ETHYLENEO } & 0.00 \\
\text { CONC. (CC/CM) } & 0.0 \\
\text { HEIGHT (KGS) } & 0\end{array}$ & $\begin{array}{r}\text { ETHANOL } \\
1.01 \\
0\end{array}$ & $\begin{array}{r}0.65 \\
0\end{array}$ & & & $\begin{array}{r}0.00 \\
0\end{array}$ & $\begin{array}{r}0.26 \\
0\end{array}$ & & $\begin{array}{r}2.49 \\
1\end{array}$ & & & & $\begin{array}{r}0.09 \\
1\end{array}$ \\
\hline $\begin{array}{l}\text { SODIUM HYPOCHLORITE } \\
\text { CONC. (CC/CM) } \\
\text { WEIGHT (KGS) }\end{array}$ & & $\begin{array}{r}2.95 \\
0\end{array}$ & & . & & & & & & & & $\begin{array}{r}0.03 \\
0\end{array}$ \\
\hline $\begin{array}{l}\text { STODDARD SOLVENT } \\
\text { CONC. (CC/CM) } \\
\text { WEIGHT (KGS) }\end{array}$ & $\begin{array}{r}6.30 \\
1\end{array}$ & $\begin{array}{r}4.05 \\
0\end{array}$ & & & 0.02 & $\begin{array}{r}1.63 \\
1\end{array}$ & & $\begin{array}{r}15.59 \\
4\end{array}$ & & & & $\begin{array}{r}0.56 \\
6\end{array}$ \\
\hline $\begin{array}{l}\text { HFEF COOLING TOWER } \\
\text { VOLUME (1000 CM) }\end{array}$ & & & 0 & 0 & 0 & 0 & 0 & 0 & 0 & 0 & 0 & 1 \\
\hline $\begin{array}{l}\text { ALKYL DIMETHYL BENZYLAMHOR } \\
\text { CONC. (CC/CM) } \\
\text { HEIGHT (KGS) }\end{array}$ & M CHLOR & & $\begin{array}{r}0.19 \\
0\end{array}$ & $\begin{array}{r}0.04 \\
0\end{array}$ & $\begin{array}{r}0.00 \\
0\end{array}$ & $\begin{array}{r}0.00 \\
0\end{array}$ & $\begin{array}{r}0.09 \\
0\end{array}$ & $\begin{array}{r}0.12 \\
0\end{array}$ & $\begin{array}{r}0.20 \\
0\end{array}$ & $\begin{array}{r}0.28 \\
0\end{array}$ & $\begin{array}{r}0.63 \\
0\end{array}$ & $\begin{array}{r}0.01 \\
0\end{array}$ \\
\hline $\begin{array}{l}\text { ALKYL METHYLBENZYL AMMONIU } \\
\text { CONC. (CC/CM) } \\
\text { HEIGHT (KGS) }\end{array}$ & HLORIDE & & $\begin{array}{r}0.19 \\
0\end{array}$ & $\begin{array}{r}0.04 \\
0\end{array}$ & $\begin{array}{r}0.00 \\
0\end{array}$ & $\begin{array}{r}0.00 \\
0\end{array}$ & $\begin{array}{r}0.09 \\
0\end{array}$ & $\begin{array}{r}0.12 \\
0\end{array}$ & $\begin{array}{r}0.20 \\
0\end{array}$ & $\begin{array}{r}0.28 \\
0\end{array}$ & $\begin{array}{r}0.63 \\
0\end{array}$ & $\begin{array}{r}0.01 \\
0\end{array}$ \\
\hline & & & & $\begin{array}{l}\text { LS MAI } \\
0 \text { IN } \\
00 \text { IN }\end{array}$ & $\begin{array}{l}\text { ADD I } \\
\text { LUMN } \\
\text { LUMN I }\end{array}$ & $\begin{array}{l}\text { TOTAL } \\
\text { ATES A } \\
\text { ATES A }\end{array}$ & $\begin{array}{l}\text { CAUSE } \\
\text { AL OF } \\
\text { AL OF }\end{array}$ & $\begin{array}{l}\text { ROUNDIN } \\
.5 \\
.005\end{array}$ & & & $\cdot$ & \\
\hline
\end{tabular}




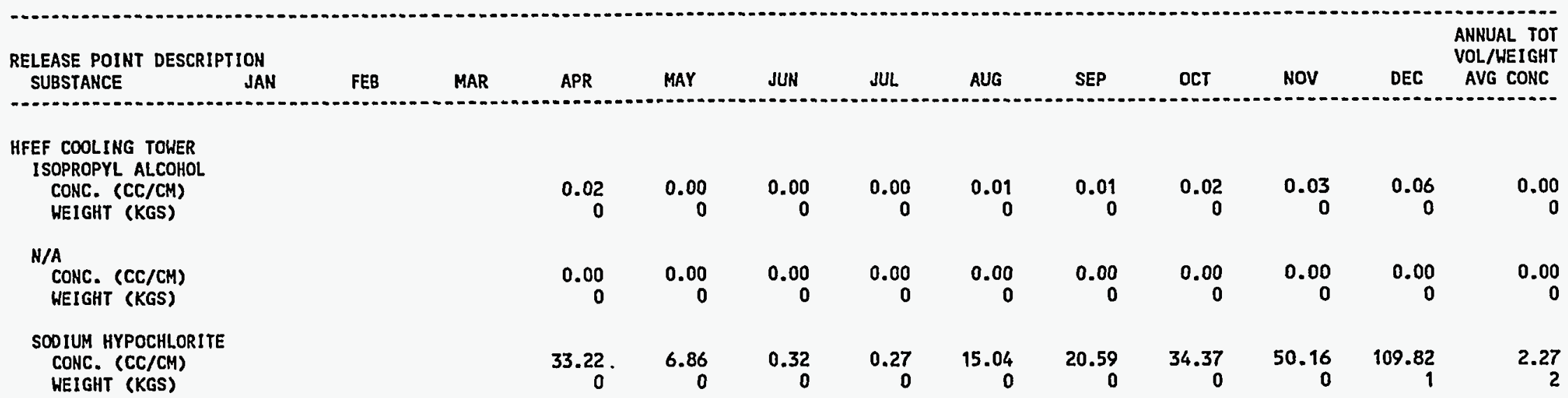


PAGE: 1

SCHED. NO. INRPTOO8A
IDAHO OPERATIONS OFFICE

$11-J U L-95$

UNITED STATES DEPARTMENT OF ENERGY

INEL NONRADIOLOGICAL HASTE MANAGEMENT INFORMATION SYSTEM
FOR JARUARY THROUGH DECEMBER 1994

ANL LIQUID DISPOSED SUBSTANCES SUMMARY

RPT 115-1

ANL

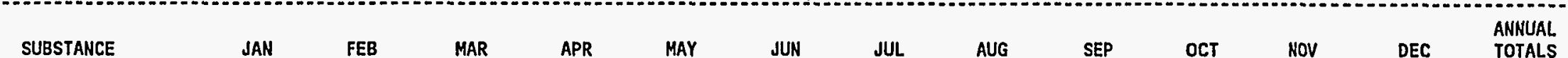

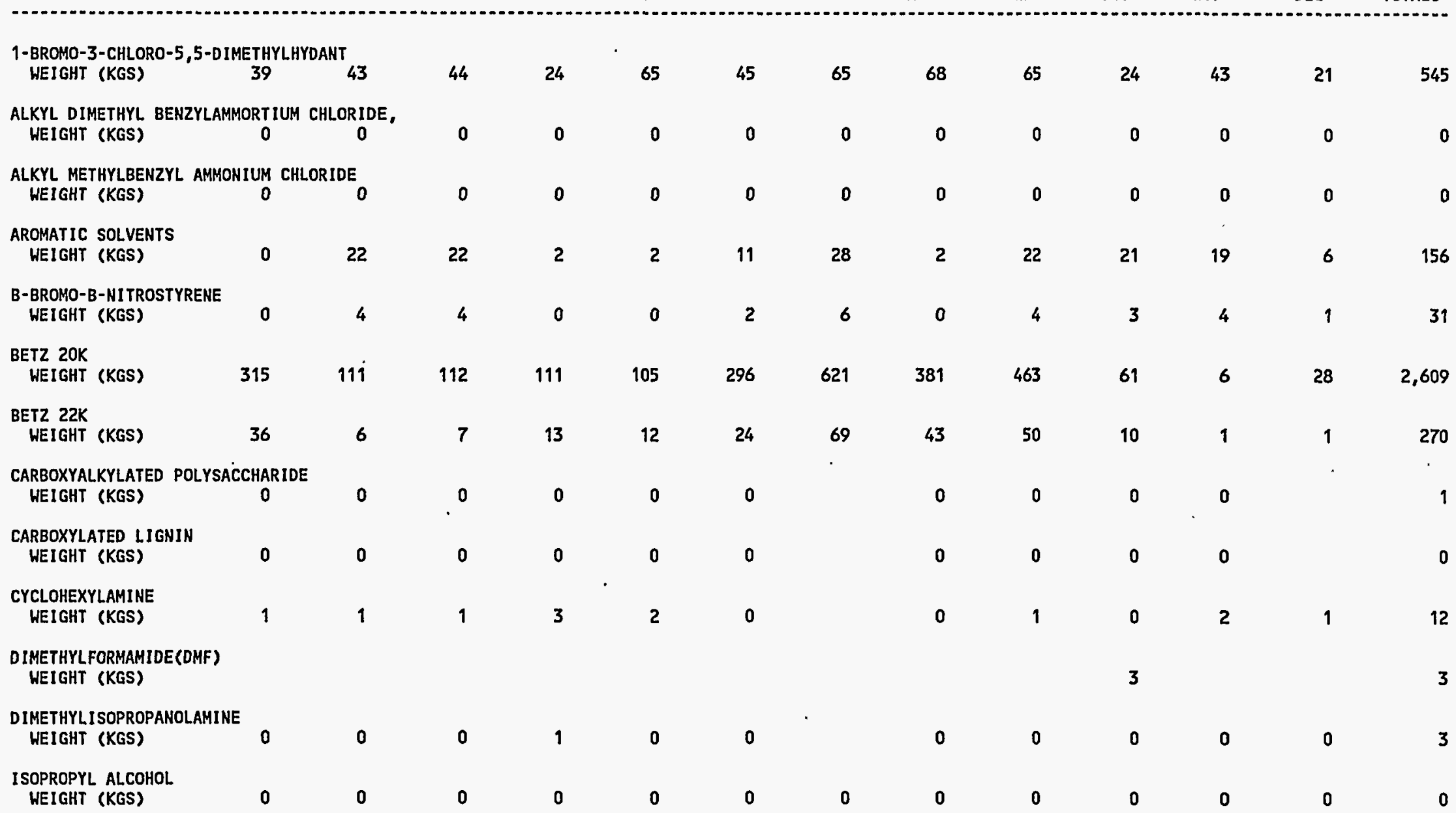

DETAILS MAY NOT ADD UP TO TOTALS BECAUSE OF ROUNDING

$O$ IN A COLUMN INDICATES A TOTAL OF $<0.5$ 
PAGE: 2

SCHED. NO. INRPTOOBA
IDAHO OPERATIONS OFFICE

11-JUL-95

UNITED STATES DEPARTMENT OF ENERGY

INEL NONRADIOLOGICAL HASTE MANAGEMENT INFORMATION SYSTEN

FOR JANUARY THROUGH DECEMBER 1994

ANL LIQUID DISPOSED SUBSTANCES SUMMARY

RPT 115-I

ANL

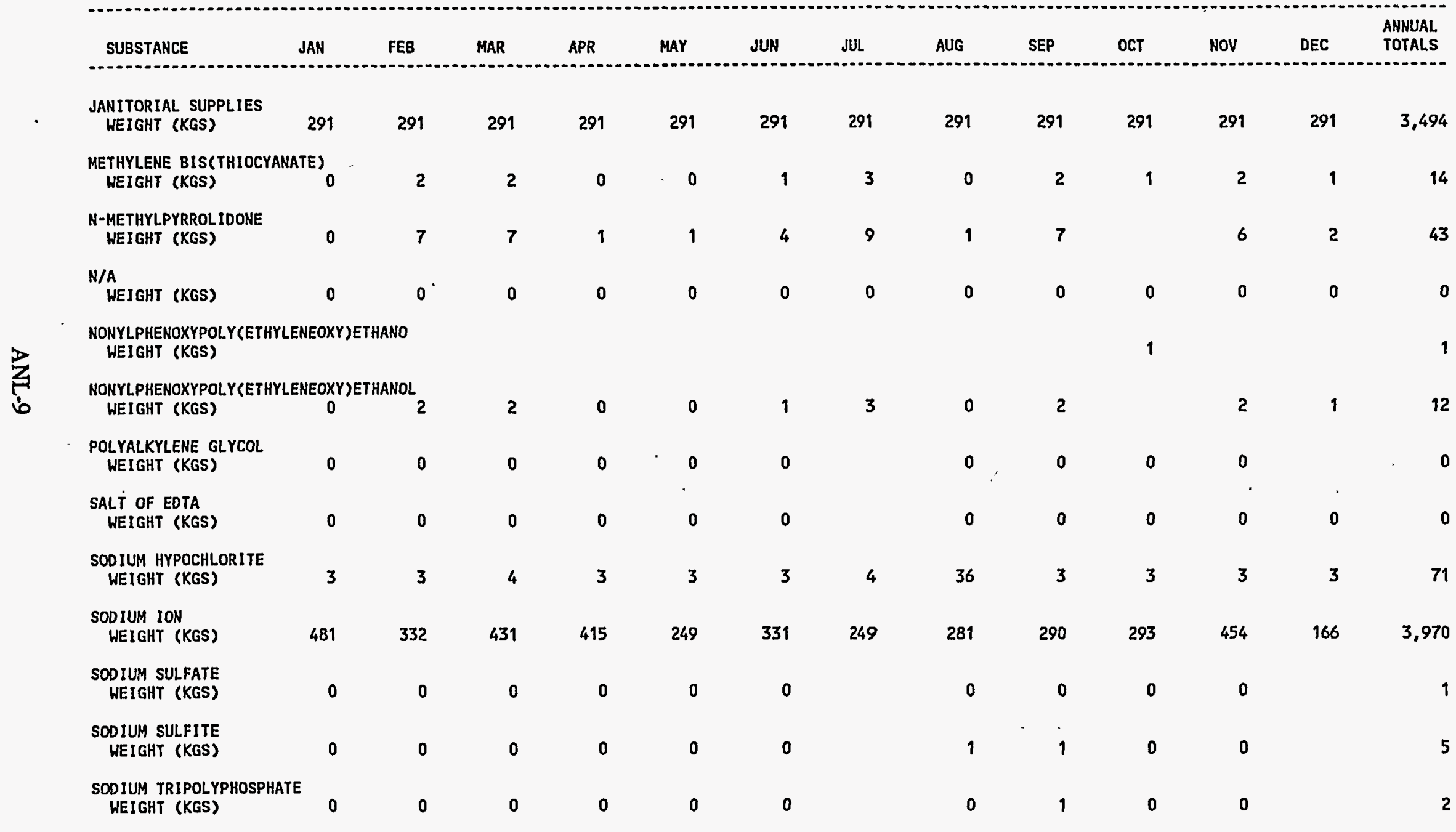

DETAILS MAY NOT ADD UP TO TOTALS BECAUSE OF ROUNDING

0 IN A COLUMN INDICATES A TOTAL OF $<0.5$ 
PAGE: 3

IDAHO OPERATIONS OFFICE

$11-J U L-95$

SCHED. NO. INRPTOOBA

UNITED STATES DEPARTMENT OF ENERG

OLOGICAL HASTE MANAGEMENT INFORMATION SYSTEM

FOR JANUARY THROUGH DECEMBER 1994

ANL LIQUID DISPOSED SUBSTANCES SUMMARY

RPT 115-

ANL

\begin{tabular}{|c|c|c|c|c|c|c|c|c|c|c|c|c|c|}
\hline SUBSTANCE & JAN & FEB & MAR & APR & MAY & JUN & JUL & AUG & SEP & ОСТ & NOV & DEC & $\begin{array}{l}\text { ANNUAL } \\
\text { TOTALS }\end{array}$ \\
\hline $\begin{array}{c}\text { STODDARD SOLVENT } \\
\text { HEIGHT (KGS) }\end{array}$ & 0 & 12 & 12 & 1 & 1 & 6 & 16 & 1 & 12 & & 11 & 4 & 77 \\
\hline $\begin{array}{l}\text { SULFATE ION } \\
\text { WEIGHT (KGS) }\end{array}$ & 5,126 & 1,670 & 3,348 & 1,225 & 966 & 2,835 & 7,257 & 5,080 & 6,215 & 935 & 966 & 901 & 36,526 \\
\hline
\end{tabular}




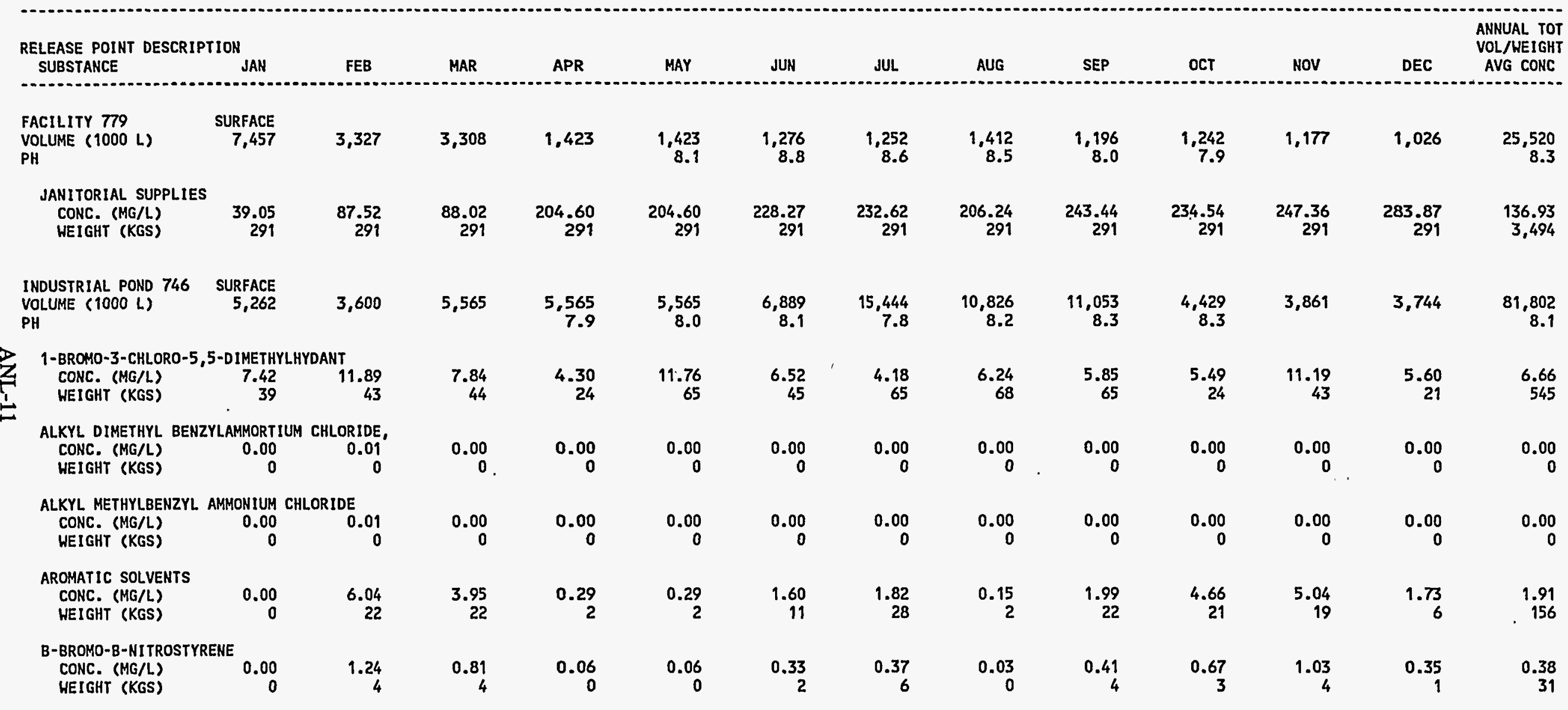


PAGE: 2

SCHED. NO. INRPTOOB
IDAHO OPERATIONS OFFICE

$11-J U L-95$

UNITED STATES DEPARTMENT OF ENERGY

INEL NONRADIOLOGICAL WASTE MANAGEMENT INFORMATION SYSTEM

FOR JANUARY THROUGH DECEMBER 1994

ANL. RELEASE POINT AND LIQUID DISPOSED SUBSTANCES SUMMARY

RPT 115-1

ANL

ANNUAL TOT

ANOLAL TOT

AVG CONC

RELEASE POINT DESCRIPTI

SUBSTANCE

JAN

FEB MAR

APR

MAY

JUN

JUL

AUG

SEP

OCT

NOV

DEC

\section{INDUSTRIAL. POND 746 SURFACE}

BETZ 2OK

CONC. (MG/L) $\quad 59.83$

$\begin{array}{rr}30.74 & 20.13 \\ 111 & 11\end{array}$

19.97
111

$\begin{array}{rr}18.91 & 42.93 \\ 105 & 296\end{array}$

40.24
621

35.19
381

41.86
463

13.83
61

1.43
6

7.41
28

31.90

BETZ 22K

CONC. (MG/L)

6.91

$\begin{array}{lll}1.67 & 1.20 & 2.26\end{array}$

$2.15 \quad 3.55$

4.45
69

$3.94 \quad 4.49$

4.49
50

2.27

0.16

0.17

3.31
270

CARBOXYALKYLATED POLYSACCHARIDE

CONC. (MG/L)

0.02

0.02

0.01

0.01

0.00

0.00
0

$\begin{array}{rr}0.01 & 0.02 \\ 0 & 0\end{array}$

0.01
.0

0.02
0

0.01
1

CARBOXYLATED LIGNIN

CONC. (MG/L)

0.00

$0.01 \quad 0.00$

0.00

0.00
0

0.00
0

0.00

0.01
0

0.00
0

0.00
0

0.00
0

CYCLOHEXYLAMINE

CONC. (MG/L)

$\begin{array}{lll}0.25 & 0.37 & 0.20\end{array}$

0.46

$0.3 \dot{1}$

0.05

0.02

0.05

0.09

0.43
2

0.16

0.14
12

DIMETHYLFORMAMIDE (DMF)

CONC. (MG/L)

WEIGHT (KGS)

DIMETHYLISOPROPANOLAMINE

CONC. (MG/L)

0.07

0.11

0.02

0.00

0.67
3

HEIGHT (KGS)

ISOPROPYL ALCOHOL

CONC. ( $M G / L)$

0.00

0.00

0.00

0.00

0.00

0.00
0

0.00
0

0.00
0

0.00
0

0.00
0

0.00

0.12

12

0.04
0

3

0.03

0.00

0.00
0

0.00
0

DETAILS MAY NOT ADD UP TO TOTALS BECAUSE OF ROUNDING

0 IN A COLUMN INDICATES A TOTAL OF $<0.5$

0.00 IN A COLUHM INDICATES A TOTAL OF $<0.005$ 
PAGE: 3

SCHED. NO. IHRPTOOS
IDAHO OPERATIONS OFFICE

11-JUL-95

UMITED STATES DEPARTMENT OF ENERGY

INEL NONRADIOLOGICAL UASTE MANAGEMENT INFORMATION SYSTEM

FOR JANUARY THROUGH DECEMBER 1994

ANL. RELEASE POINT AND LIQUID DISPOSED SUBSTANCES SUMMARY

RPT 115-I

ANL

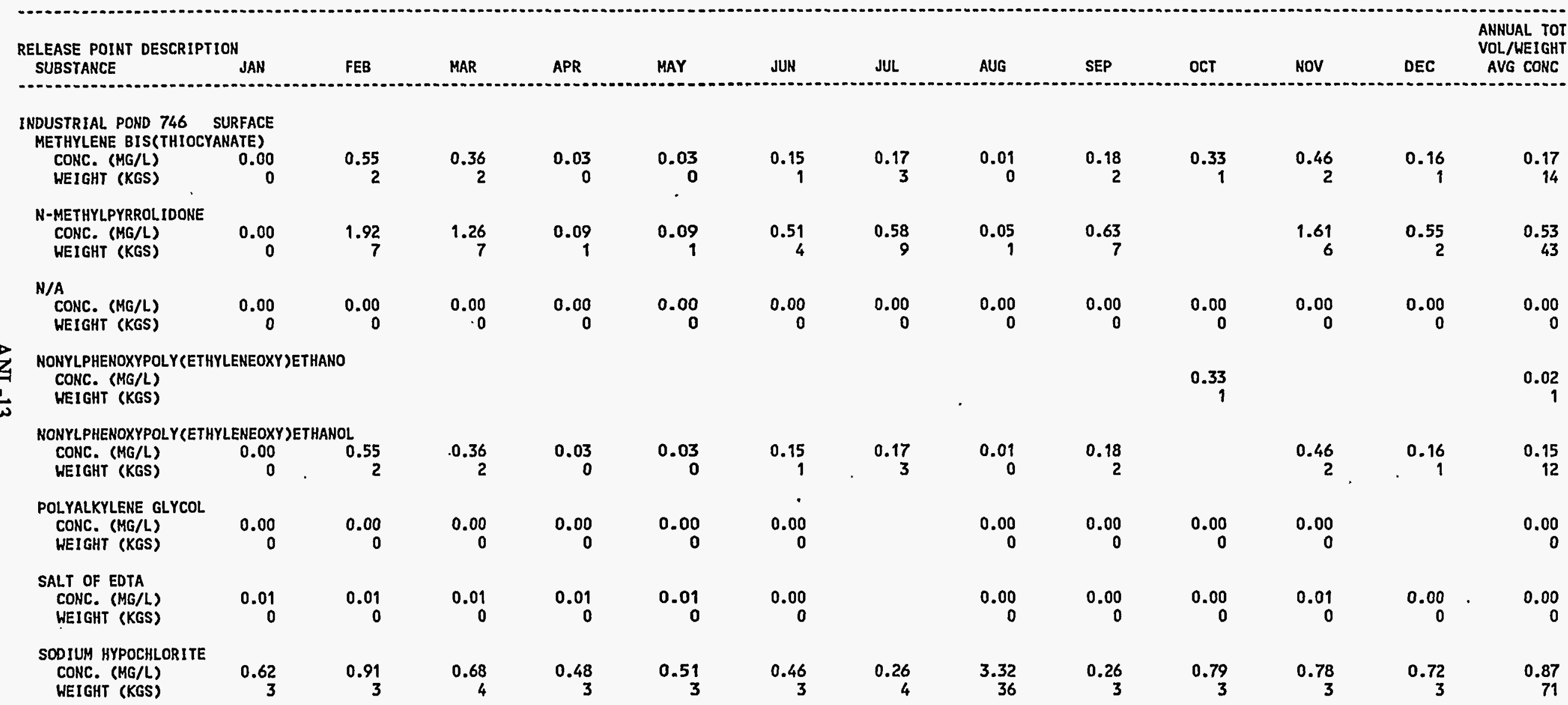

DETAILS MAY NOT ADD UP TO TOTALS BECAUSE OF ROUNDING

$\begin{aligned} & 0 \text { IN A COLUMN INDICATES A TOTAL OF }<0.5 \\ & 0.00 \text { IN A COLUMN INDICATES A TOTAL OF }<0.005\end{aligned}$ 


\begin{tabular}{|c|c|c|c|c|c|c|c|c|c|c|c|c|}
\hline $\begin{array}{l}\text { RELEASE POINT DESCRIPTION } \\
\text { SUBSTANCE }\end{array}$ & FEB & MAR & APR & MAY & JUN & JUL & AUG & SEP & ОСТ & NOV & DEC & $\begin{array}{c}\text { ANNUAL TOT } \\
\text { VOL/WEIGHT } \\
\text { AVG CONC }\end{array}$ \\
\hline $\begin{array}{l}\text { INDUSTRIAL POND } 746 \text { SURFACE } \\
\text { SODIUM ION }\end{array}$ & & & & & & & & & & & & \\
\hline $\begin{array}{l}\text { CONC. (MG/L) } \\
\text { WEIGHT (KGS) }\end{array}$ & $\begin{array}{r}92.10 \\
332\end{array}$ & $\begin{array}{r}77.38 \\
431\end{array}$ & $\begin{array}{r}74.50 \\
415\end{array}$ & $\begin{array}{r}44.68 \\
249\end{array}$ & $\begin{array}{r}48.09 \\
331\end{array}$ & $\begin{array}{r}16.09 \\
249\end{array}$ & $\begin{array}{r}25.99 \\
281\end{array}$ & $\begin{array}{r}26.26 \\
290\end{array}$ & $\begin{array}{r}66.06 \\
293\end{array}$ & $\begin{array}{r}117.49 \\
454\end{array}$ & $\begin{array}{r}44.25 \\
166\end{array}$ & $\begin{array}{l}48.53 \\
3,970\end{array}$ \\
\hline $\begin{array}{l}\text { SODIUM SULFATE } \\
\text { CONC. (MG/L) } \\
\text { WEIGHT (KGS) }\end{array}$ & $\begin{array}{r}0.02 \\
0\end{array}$ & $\begin{array}{r}0.01 \\
0\end{array}$ & $\begin{array}{r}0.01 \\
0\end{array}$ & $\begin{array}{r}0.00 \\
0\end{array}$ & $\begin{array}{r}0.00 \\
0\end{array}$ & & $\begin{array}{r}0.01 \\
0\end{array}$ & $\begin{array}{r}0.02 \\
0\end{array}$ & $\begin{array}{r}0.01 \\
0\end{array}$ & $\begin{array}{r}0.02 \\
0\end{array}$ & & $\begin{array}{r}0.01 \\
1\end{array}$ \\
\hline $\begin{array}{l}\text { SODIUM SULFITE } \\
\text { CONC. (MG/L) } \\
\text { WEIGHT (KGS) }\end{array}$ & $\begin{array}{r}0.12 \\
0\end{array}$ & $\begin{array}{r}0.05 \\
0\end{array}$ & $\begin{array}{r}0.07 \\
0\end{array}$ & $\begin{array}{r}0.02 \\
0\end{array}$ & $\begin{array}{r}0.03 \\
0\end{array}$ & & $\begin{array}{r}0.06 \\
1\end{array}$ & $\begin{array}{r}0.13 \\
1\end{array}$ & $\begin{array}{r}0.06 \\
0\end{array}$ & $\begin{array}{r}0.09 \\
0\end{array}$ & & $\begin{array}{r}0.06 \\
5\end{array}$ \\
\hline $\begin{array}{lr}\text { SODIUM TRIPOLYPHOSPHATE } \\
\text { CONC. (MG/L) } & 0.03 \\
\text { WEIGHT (KGS) } & 0\end{array}$ & $\begin{array}{r}0.04 \\
0\end{array}$ & $\begin{array}{r}0.02 \\
0\end{array}$ & $\begin{array}{r}0.03 \\
0\end{array}$ & $\begin{array}{r}0.01 . \\
0\end{array}$ & $\begin{array}{r}0.01 \\
0\end{array}$ & & $\begin{array}{r}0.02 \\
0\end{array}$ & $\begin{array}{r}0.05 \\
1\end{array}$ & $\begin{array}{r}0.02 \\
0\end{array}$ & $\begin{array}{r}0.03 \\
0\end{array}$ & & $\begin{array}{r}0.02 \\
2\end{array}$ \\
\hline $\begin{array}{l}\text { STODDARD SOLVENT } \\
\text { CONC. (MG/L) } \\
\text { HEIGHT (KGS) }\end{array}$ & $\begin{array}{r}3.43 \\
12\end{array}$ & $\begin{array}{r}2.24 \\
.12\end{array}$ & $\begin{array}{r}0.17 \\
1\end{array}$ & $\begin{array}{r}0.17 \\
1\end{array}$ & $\begin{array}{r}0.91 \\
6\end{array}$ & $\begin{array}{r}1.04 \\
16\end{array}$ & $\begin{array}{r}0.09 \\
.1\end{array}$ & $\begin{array}{r}1.13 \\
12\end{array}$ & & $\begin{array}{r}2.87 \\
11\end{array}$ & $\begin{array}{r}0.98 \\
4\end{array}$ & $\begin{array}{r}0.94 \\
77\end{array}$ \\
\hline $\begin{array}{l}\text { SULFATE ION } \\
\text { CONC. (MG/L) } \\
\text { WEIGHT (KGS) }\end{array}$ & $\begin{array}{r}463.89 \\
1,670\end{array}$ & $\begin{array}{r}601.61 \\
3,348\end{array}$ & $\begin{array}{r}220.23 \\
1,225\end{array}$ & $\begin{array}{r}173.65 \\
966\end{array}$ & $\begin{array}{r}411.53 \\
2,835\end{array}$ & $\begin{array}{r}469.91 \\
7,257\end{array}$ & $\begin{array}{r}469.27 \\
5,080\end{array}$ & $\begin{array}{r}562.27 \\
6,215\end{array}$ & $\begin{array}{r}211.15 \\
935\end{array}$ & $\begin{array}{r}250.25 \\
966\end{array}$ & $\begin{array}{r}240.80 \\
901\end{array}$ & $\begin{array}{l}446.52 \\
36,526\end{array}$ \\
\hline
\end{tabular}


PAGE: 9

SCHED. NO. INRPTOOS

IDAHO OPERATIONS OFFICE

10-JUL-95

UNITEO STATES DEPARTHENT OF ENERGY

WASTE MANAGEMENT INFORMATION SYSTEM

FOR JANUARY THROUGH DECEMBER 1994

ANL LANDFILL SOLID DISPOSED HASTE SUMMARY

RPT 116-1

RPT

(ALL VOLUMES = CUBIC METERS)

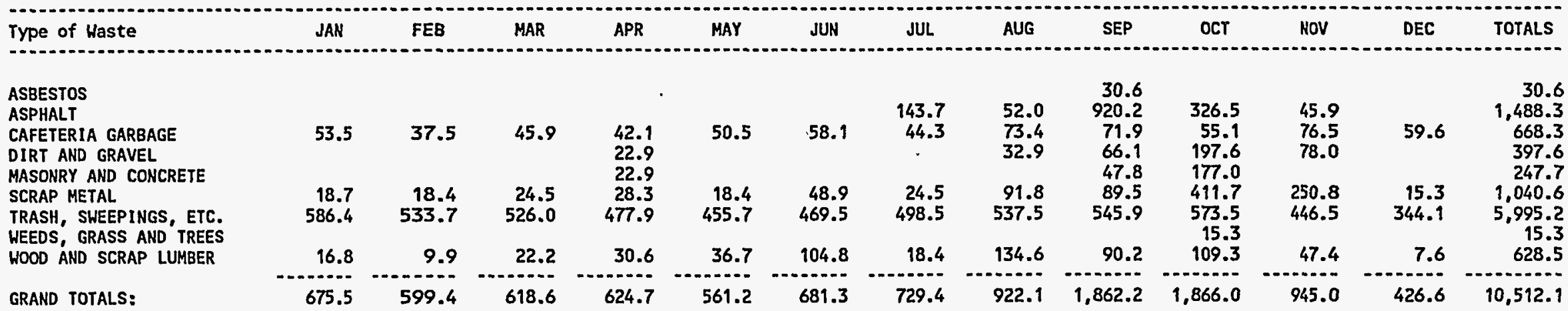

$\frac{3}{3}$ 
FOR JANUARY THROUGH DECEMBER 1994

ANL. HATER USAGE AND DISPOSAL SUMMARY

(ALL VOLUMES = NEAREST THOUSAND LITERS )

\section{WELL OR BLDG. NO./} SEWAGE BLDG. NO.

JAN

FEB MAR APR

MAY

JUN JUL AUG

SEP

OCT

Nov

DEC AVG CONC

WATER DATA

001 HATER PUMPED (L)
002 WATER PUMPED (L)

\begin{tabular}{rrr}
23,659 & 5,565 & 14,460 \\
23,659 & 5,565 & 14,460 \\
\hline 47,318 & 11,129 & 28,920
\end{tabular}

\begin{tabular}{rrr}
7,041 & 8,934 & 16,126 \\
7,079 & 8,934 & 16,126 \\
\hline 14,120 & 17,867 & 32,252
\end{tabular}

\section{$\begin{array}{lll}37,854 & 27,217 \quad 29,072\end{array}$}

\begin{tabular}{rrrr}
7,003 & 4,845 & 4,648 & 186,423 \\
7,003 & 4,883 & 4,648 & 186,499 \\
\hline 14,006 & 9,728 & 9,297 & 372,922
\end{tabular}

WATER DISPOSED

AIR (L)
SURFACE (L)
SUBSURFACE (L)

$\begin{array}{rrr}21,085 & 2,430 & 9,388 \\ 12,719 & 6,927 & 8,858 \\ 83 & 83 & 83\end{array}$

111
5,905
83

522
5,678
83

$\begin{array}{rr}10,751 & 43,532 \\ 8,176 & 16,694\end{array}$

54,434

58,144

$617-1,461$

$9,297 \quad 372,922$

SUBSURFACE (L)

SEWAGE DATA

$\begin{array}{rr}617 & 1,461 \\ 5,678 & 5,035\end{array}$

579
4,770

146,386

BIOCHEMICAL OXYGEN DEMAND (BOD)

RAW CONC. (MG/L)

FINAL CONC. (MG/L)

$\%$ REMOVED

DISSOLVED OXYGEN (DO)

RAW CONC. (MG/L)

FINAL CONC. (MG/L)

INCREASED FACTOR

\section{SETTLEABLE SOLIDS (SS)}

RAW CONC. (ML/L)

FINAL CONC. (ML/L)

\% REMOVED

PH FINAL 
PAGE: 2

SCHED. NO. INRPT010
IDAHO OPERATIONS OFFICE

$11-J U L-95$

UNITED STATES DEPARTMENT OF ENERGY

INEL NONRADIOLOGICAL WASTE MANAGEMENT INFORMATION SYSTEH

FOR JANUARY THROUGH DECEMBER 1994

ANL WATER USAGE AND DISPOSAL SUMMARY

RPT 117-1

ANL

(ALL VOLUMES = NEAREST THOUSAND LITERS)

ANNUAL TOT

WELL OR BLDG. NO.I

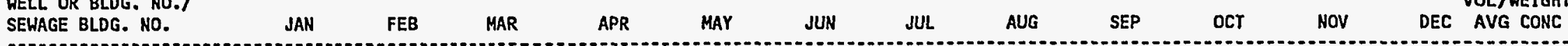

39

39

39

39

39

39

39

39

39

39

39

39

468

BIOCHEMICAL OXYGEN DEMAND (BOD)

RAH CONC. (MG/L)

FINAL CONC. (MG/L)

\% REMOVED

DISSOLVED OXYGEN (DO)

RAW CONC. (MG/L)

FINAL CONC. (MG/L)

INCREASED FACTOR

\section{SETTLEABLE SOLIDS (SS)
RAW CONC. (ML/L) \\ FINAL CONC. (ML/L) \\ \% REMOVED}

PH FINAL

DETAILS MAY NOT ADD UP TO TOTALS BECAUSE OF ROUNDING

0 IN A COLUMN INDICATES A TOTAL OF $<0.5$

0.0 IN A COLUMN INDICATES A TOTAL OF $<0.05$

0.00 IN A COLUMN INDICATES A TOTAL OF $<0.005$ 
PAGE: $\quad 3$

SCHED. NO. INRPT010

IDAHO OPERATIONS OFFICE

UNITED STATES DEPARTMENT OF ENERGY

$11-J U L-95$

INEL NONRADIOLOGICAL. HASTE MANAGEMENT INFORMATION SYSTEM

FOR JANUARY THROUGH DECEMBER 1994

ANL WATER USAGE AND DISPOSAL. SUMMARY

RPT 117-

(ALL VOLUMES = NEAREST THOUSAND LITERS)

ANL

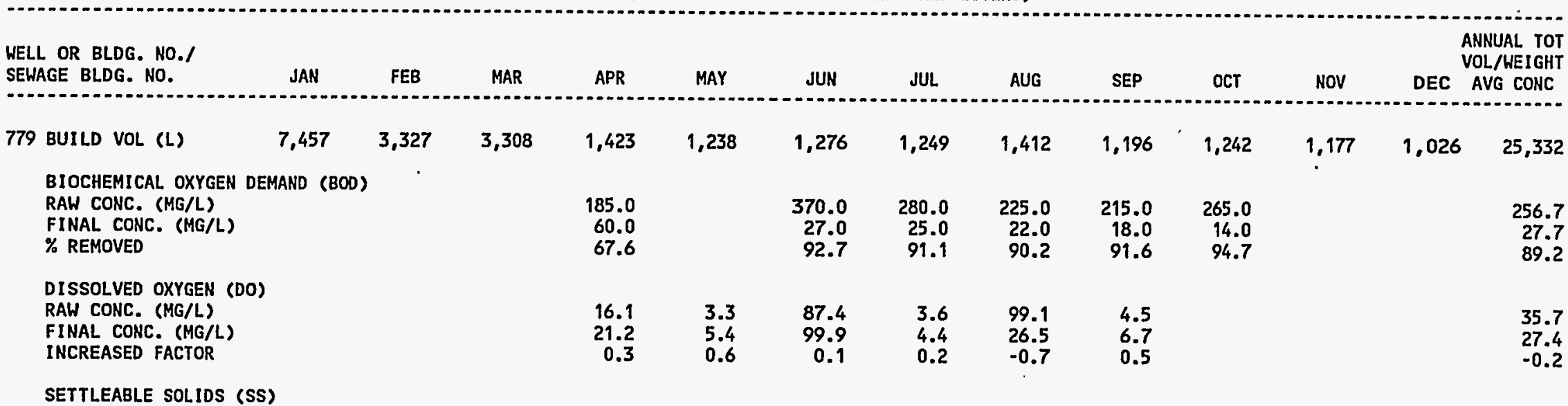

SETTLEABLE SOLIDS (SS
RAH CONC. (ML/L)
FINAL CONC. (ML/L)
$\%$ REMOVED

PH FINAL. 
PAGE: 4

SCHED. NO. INRPT010

IDAHO OPERATIONS OFFICE

11-JUL-95

UNITED STATES DEPARTMENT OF ENERGY

INEL NONRADIOLOGICAL HASTE MANAGEMENT INFORMATION SYSTEH

FOR JANUARY THROUGH DECEMBER 1994

ANL. HATER USAGE AND DISPOSAL SUMMARY

RPT 117-I

(ALL VOLUMES = NEAREST THOUSAND LITERS)

WELL OR BLDG. NO.

HELL OR BLDG. NO./
SEWAGE BLOG. NO. JAN

FEB MAR APR MAY JUN JUL AUG

SEP

793 BUILD VOL. (L)

8

8

8

8

8

8

8

8

8

OCT NOV

DEC AVG CONC

BIOCHEMICAL OXYGEN DEMAND (BOD)

RAH CONC. (MG/L)

FINAL CONC. (MG/L)

\% REMOVED

DISSOLVED OXYGEN (DO)

RAW CONC. (MG/L)

FINAL CONC. (MG/L)

INCREASED FACTOR

SETTLEABLE SOLIDS (SS)
RAW CONC. (ML/L)
FINAL CONC. (ML/L)
\% REMOVED
PH FINAL

TOTAL SEWAGE(L)

3,411

TOTAL WATER DISPOSED(L) $\quad 41,428$

12,852

3,392

1,507

$\begin{array}{rrr}1,321 . & 1,359 & 1,333 \\ 7,605 & 20,370 & 61,642\end{array}$

1,495

1,280

$\begin{array}{rr}1,325 & 1,261 \\ 7,704 & 7,840 \\ 55.0 & 80.6\end{array}$

$1,109 \quad 26,334$

ACCOUNTABILITY (\%)

87.6

$115.5 \quad 75.1$

$\begin{array}{llll}53.9 & 42.6 & 63.2 & 81.4\end{array}$

79.1

69.3

55.0

80.6

$6,541 \quad 278,651$

DETAILS MAY NOT ADD UP TO TOTALS BECAUSE OF ROUNDING

0 IN A COLUMN INDICATES A TOTAL OF $<0.5$

0.0 IN A COLUMN INDICATES A TOTAL OF $<0.05$

0.00 IN A COLUMN INDICATES A TOTAL OF $<0.005$ 
PAGE: 1

IDAHO OPERATIONS OFFICE

24-MAR-95

SCHED. NO. INRPTOII

UHITED STATES DEPARTMENT OF ENERGY

INEL MOHRADIOLOGICAL. WASTE MANAGEMENT INFORMATION SYSTEM

FOR JANUARY THROUGH DECEMBER 1994

ANL. FUEL OIL USAGE AND STACK EFFLUENTS SUMMARY

RPT 118-I

ANL.

(ALL VALUES = NEAREST THOUSAND)

\begin{tabular}{|c|c|c|c|c|c|c|c|c|c|c|c|c|c|}
\hline $\begin{array}{c}\text { FUEL OIL TYPE } \\
\text { EFFLUENTS }\end{array}$ & JAN & FEB & MAR & APR & MAY & JUN & JUL & AUG & SEP & OCT & NOV & DEC & $\begin{array}{l}\text { ANNUAL } \\
\text { TOTALS }\end{array}$ \\
\hline $\begin{array}{l}\text { TYPE } 2 \\
\text { VOLUME (LITERS) } \\
\text { SO2 (KGS) } \\
\text { NOX (KGS) }\end{array}$ & $\begin{array}{r}175 \\
1\end{array}$ & $\begin{array}{r}262 \\
1\end{array}$ & $\begin{array}{r}237 \\
2\end{array}$ & $\begin{array}{r}231 \\
2\end{array}$ & $\begin{array}{r}204 \\
1\end{array}$ & $\begin{array}{r}152 \\
1\end{array}$ & $\begin{array}{l}4 \\
0\end{array}$ & $\begin{array}{r}26 \\
0\end{array}$ & $\begin{array}{r}51 \\
0\end{array}$ & $\begin{array}{r}233 \\
2\end{array}$ & $\begin{array}{r}335 \\
2\end{array}$ & $\begin{array}{r}357 \\
2\end{array}$ & $\begin{array}{r}2,267 \\
14\end{array}$ \\
\hline PARTICULATES (KGS) & 0 & 0 & 0 & $\mathbf{0}$ & 0 & 0 & 0 & 0 & 0 & 0 & 0 & $\mathbf{0}$ & $\mathbf{0}$ \\
\hline $\begin{array}{l}\text { DIESEL } \\
\text { VOLUME (LITERS) } \\
\text { SO2 (KGS) } \\
\text { NOX (KGS) } \\
\text { PARTICULATES (KGS) }\end{array}$ & & & & & - & & & & . & & $\begin{array}{l}0 \\
0\end{array}$ & $\begin{array}{l}1 \\
0\end{array}$ & $\begin{array}{l}1 \\
0\end{array}$ \\
\hline $\begin{array}{l}\text { GRAND TOTALS: } \\
\text { VOLUME (LITERS) } \\
\text { SO2 (KGS) } \\
\text { NOX (KGS) }\end{array}$ & $\begin{array}{r}175 \\
1\end{array}$ & $\begin{array}{r}262 \\
1\end{array}$ & $\begin{array}{r}237 \\
2\end{array}$ & $\begin{array}{r}231 \\
2\end{array}$ & $\begin{array}{r}204 \\
1\end{array}$ & $\begin{array}{r}152 \\
1\end{array}$ & $\begin{array}{l}4 \\
0\end{array}$ & $\begin{array}{r}26 \\
0\end{array}$ & $\begin{array}{r}51 \\
0\end{array}$ & $\begin{array}{r}233 \\
2\end{array}$ & $\begin{array}{r}335 \\
2\end{array}$ & $\begin{array}{r}358 \\
2\end{array}$ & $\begin{array}{r}2,268 \\
14\end{array}$ \\
\hline PARTICULATES (KGS) & 0 & 0 & $\mathbf{0}$ & 0 & 0 & 0 & 0 & 0 & 0 & $\mathbf{0}$ & 0 & 0 & 0 \\
\hline
\end{tabular}




\section{Central Facilities Area 1994 Detail Data}

Report 135-CFA Liquid Disposed Substances Summary . . . . . . . . . . CFA-3

Report 135-CFA Release Point And Liquid Disposed Substances Summary . . . CFA-5

Report 136-CFA Landfill Solid Disposed Waste Summary . . . . . . . . . . . . CFA-8

Report 137-CFA Water Usage And Disposal Summary . . . . . . . . . . . . . CFA-9

Report 138-CFA Fuel Oil Usage And Stack Effluents Summary . . . . . . . . CFA-11 


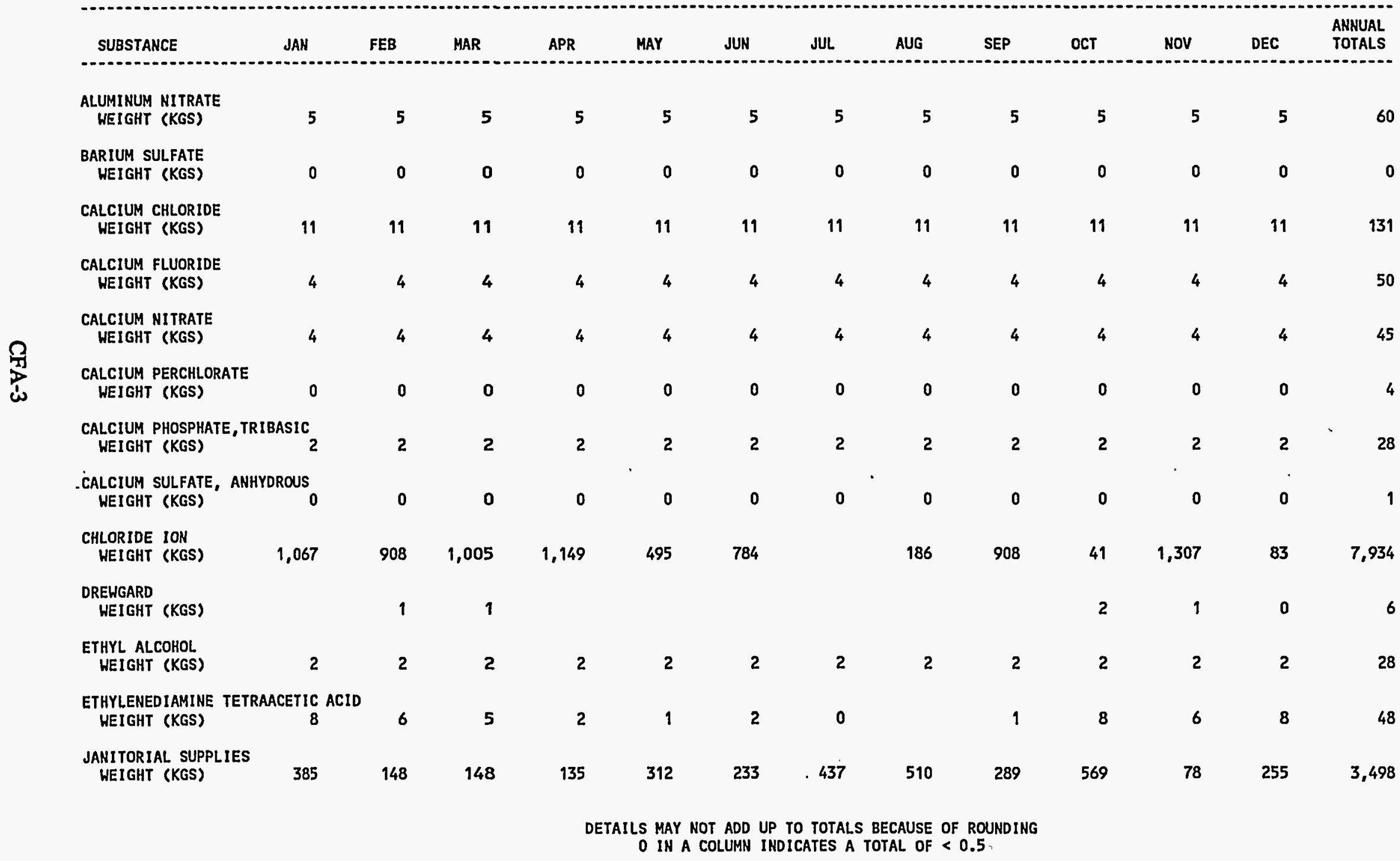


PAGE: 2

IDAHO OPERATIONS OFFICE

13-JUL-95

SCHED. NO. INRPTOOBA

UNITED STATES DEPARTMENT OF ENERGY

INEL NONRADIOLOGICAL HASTE MANAGEMENT INFORMATION SYSTEM

FOR JANUARY THROUGH DECEMBER 1994

CFA LIQUID DISPOSED SUBSTANCES SUMMARY

RPT 135-I

CFA

\begin{tabular}{|c|c|c|c|c|c|c|c|c|c|c|c|c|c|}
\hline SUBSTANCE & JAN & FEB & MAR & APR & MAY & JUN & JUL & AUG & SEP & ОСТ & NoV & DEC & $\begin{array}{l}\text { ANNUAL } \\
\text { TOTALS }\end{array}$ \\
\hline $\begin{array}{l}\text { POTASSIUM HYDROXIDE } \\
\text { WEIGHT (KGS) }\end{array}$ & 8 & 6 & 5 & 2 & 1 & 2 & 0 & & 1 & 8 & 6 & 8 & 48 \\
\hline $\begin{array}{l}\text { SODIUM ION } \\
\text { HEIGHT (KGS) }\end{array}$ & 712 & 608 & 673 & 752 & 324 & 514 & 4 & 120 & 592 & 43 & 866 & 79 & 5,288 \\
\hline $\begin{array}{l}\text { SODIUM LIGNOSULFONATE } \\
\text { WEIGHT (KGS) }\end{array}$ & 8 & 6 & 5 & 2 & 1 & 2 & 0 & & 1 & 8 & 6 & 8 & 48 \\
\hline $\begin{array}{l}\text { SULFITE ION } \\
\text { HEIGHT (KGS) }\end{array}$ & 35 & 30 & 37 & 13 & 5 & 9 & 6 & & 6 & 28 & 32 & 42 & 243 \\
\hline
\end{tabular}

0 IN A COLUMN INDICATES A TOTAL OF $<0.5$ 


\begin{tabular}{|c|c|c|c|c|c|c|c|c|c|c|c|c|}
\hline $\begin{array}{l}\text { RELEASE POINT DESCRIPTION } \\
\text { SUBSTANCE JAN }\end{array}$ & FEB & MAR & APR & MAY & JUN & JUL & AUE & SEP & OCT & NOV & DEC & $\begin{array}{l}\text { ANNUAL TOT } \\
\text { VOL/HEIGHT } \\
\text { AVG CONC }\end{array}$ \\
\hline $\begin{array}{l}\text { SEHAGE PLANT } \\
\text { VOLUME }(1000 \mathrm{~L}) \\
\text { PH }\end{array}$ & 7,946 & 11,375 & 11,386 & 13,968 & 16,979 & 17,430 & 19,029 & 14,797 & 13,665 & 9,766 & 10,375 & 155,784 \\
\hline $\begin{array}{l}\text { ALUMINUM NITRATE } \\
\text { CONC. (MG/L) } \\
\text { WEIGHT (KGS) }\end{array}$ & $\begin{array}{r}0.63 \\
5\end{array}$ & $\begin{array}{r}0.44 \\
5\end{array}$ & $\begin{array}{r}0.44 \\
5\end{array}$ & $\begin{array}{r}0.36 \\
5\end{array}$ & $\begin{array}{r}0.29 \\
5\end{array}$ & $\begin{array}{r}0.29 \\
5\end{array}$ & $\begin{array}{r}0.26 \\
5\end{array}$ & $\begin{array}{r}0.34 \\
5\end{array}$ & $\begin{array}{r}0.37 \\
5\end{array}$ & $\begin{array}{r}0.51 \\
5\end{array}$ & $\begin{array}{r}0.48 \\
5\end{array}$ & $\begin{array}{r}0.39 \\
60\end{array}$ \\
\hline $\begin{array}{l}\text { BARIUM SULFATE } \\
\text { CONC. (MG/L) } \\
\text { WEIGHT (KGS) }\end{array}$ & $\begin{array}{r}0.00 \\
0\end{array}$ & $\begin{array}{r}0.00 \\
0\end{array}$ & $\begin{array}{r}0.00 \\
0\end{array}$ & $\begin{array}{r}0.00 \\
0\end{array}$ & $\begin{array}{r}0.00 \\
0\end{array}$ & $\begin{array}{r}0.00 \\
0\end{array}$ & $\begin{array}{r}0.00 \\
0\end{array}$ & $\begin{array}{r}0.00 \\
0\end{array}$ & $\begin{array}{r}0.00 \\
0\end{array}$ & $\begin{array}{r}0.00 \\
0\end{array}$ & $\begin{array}{r}0.00 \\
0\end{array}$ & $\begin{array}{r}0.00 \\
0\end{array}$ \\
\hline $\begin{array}{l}\text { CALCIUM CHLORIDE } \\
\text { CONC. (MG/L) } \\
\text { WEIGHT (KGS) }\end{array}$ & $\begin{array}{r}1.37 \\
11\end{array}$ & $\begin{array}{r}0.96 \\
11\end{array}$ & $\begin{array}{r}0.96 \\
11\end{array}$ & $\begin{array}{r}0.78 \\
11\end{array}$ & $\begin{array}{r}0.64 \\
\quad 11\end{array}$ & $\begin{array}{r}0.62 \\
11\end{array}$ & $\begin{array}{r}0.57 \\
11\end{array}$ & $\begin{array}{r}0.74 \\
11\end{array}$ & $\begin{array}{r}0.80 \\
11\end{array}$ & $\begin{array}{r}1.11 \\
11\end{array}$ & $\begin{array}{r}1.05 \\
11\end{array}$ & $\begin{array}{r}0.84 \\
131\end{array}$ \\
\hline $\begin{array}{l}\text { CALCIUM FLUORIDE } \\
\text { CONC. (MG/L) } \\
\text { WEIGHT (KGS) }\end{array}$ & $\begin{array}{r}0.52 \\
4\end{array}$ & $\begin{array}{r}0.36 \\
4\end{array}$ & $\begin{array}{r}0.36 \\
4\end{array}$ & $\begin{array}{r}0.30 \\
4\end{array}$ & $\begin{array}{r}0.24 \\
4\end{array}$ & $\begin{array}{r}0.24 \\
4\end{array}$ & $\begin{array}{r}0.22 \\
4\end{array}$ & $\begin{array}{r}0.28 \\
4\end{array}$ & $\begin{array}{r}0.30 \\
4\end{array}$ & $\begin{array}{r}0.42 \\
4\end{array}$ & $\begin{array}{r}0.40 \\
4\end{array}$ & $\begin{array}{r}0.32 \\
50\end{array}$ \\
\hline $\begin{array}{l}\text { CALCIUM NITRATE } \\
\text { CONC. (MG/L) } \\
\text { WEIGHT (KGS) }\end{array}$ & $\begin{array}{r}0.47 \\
4\end{array}$ & $\begin{array}{r}0.33 \\
4\end{array}$ & $\begin{array}{r}0.33 \\
4\end{array}$ & $\begin{array}{r}0.27 \\
4\end{array}$ & $\begin{array}{r}0.22 \\
4\end{array}$ & $\begin{array}{r}0.22 \\
4\end{array}$ & $\begin{array}{r}0.20 \\
4\end{array}$ & $\begin{array}{r}0.25 \\
4\end{array}$ & $\begin{array}{r}0.28 \\
4\end{array}$ & $\begin{array}{r}0.39 \\
4\end{array}$ & $\begin{array}{r}0.36 \\
4\end{array}$ & $\begin{array}{r}0.29 \\
45\end{array}$ \\
\hline $\begin{array}{l}\text { CALCIUM PERCHLORATE } \\
\text { CONC. (MG/L) } \\
\text { WEIGHT (KGS) }\end{array}$ & $\begin{array}{r}0.05 \\
0\end{array}$ & $\begin{array}{r}0.03 \\
0\end{array}$ & $\begin{array}{r}0.03 \\
0\end{array}$ & $\begin{array}{r}0.03 \\
0\end{array}$ & $\begin{array}{r}0.02 \\
0\end{array}$ & $\begin{array}{r}0.02 \\
0\end{array}$ & $\begin{array}{r}0.02 \\
0\end{array}$ & $\begin{array}{r}0.02 \\
0\end{array}$ & $\begin{array}{r}0.03 \\
0\end{array}$ & $\begin{array}{r}0.04 \\
0\end{array}$ & $\begin{array}{r}0.03 \\
0\end{array}$ & $\begin{array}{r}0.03 \\
4\end{array}$ \\
\hline $\begin{array}{cr}\text { CALCIUM PHOSPHATE, TRIBASIC } \\
\text { CONC. (MG/L) } & 0.26 \\
\text { WEIGHT (KGS) } & 2\end{array}$ & $\begin{array}{r}0.30 \\
2\end{array}$ & $\begin{array}{r}0.21 \\
2\end{array}$ & $\begin{array}{r}0.21 \\
2\end{array}$ & $\begin{array}{r}0.17 \\
2\end{array}$ & $\begin{array}{r}0.14 \\
2\end{array}$ & $\begin{array}{r}0.14 \\
2\end{array}$ & $\begin{array}{r}0.12 \\
2\end{array}$ & $\begin{array}{r}0.16 \\
2\end{array}$ & $\begin{array}{r}0.17 \\
2\end{array}$ & $\begin{array}{r}0.24 \\
2\end{array}$ & $\begin{array}{r}0.23 \\
2\end{array}$ & $\begin{array}{r}0.18 \\
28\end{array}$ \\
\hline $\begin{array}{cr}\text { CALCIUM SULFATE, } & \text { ANHYDROUS } \\
\text { CONC. (HG/L) } & 0.01 \\
\text { HEIGHT (KGS) } & 0\end{array}$ & $\begin{array}{r}0.01 \\
0\end{array}$ & $\begin{array}{r}0.01 \\
0\end{array}$ & $\begin{array}{r}0.01 \\
0\end{array}$ & $\begin{array}{r}0.01 \\
0\end{array}$ & $\begin{array}{r}0.01 \\
0\end{array}$ & $\begin{array}{r}0.01 \\
0\end{array}$ & $\begin{array}{r}0.00 \\
0\end{array}$ & $\begin{array}{r}0.01 \\
0\end{array}$ & $\begin{array}{r}0.01 \\
0\end{array}$ & $\begin{array}{r}0.01 \\
0\end{array}$ & $\begin{array}{r}0.01 \\
0\end{array}$ & $\begin{array}{r}0.01 \\
1\end{array}$ \\
\hline
\end{tabular}

DETAILS MAY NOT ADD UP TO TOTALS BECAUSE OF ROUNDING

0 IN A COLUMN INDICATES A TOTAL OF $<0.5$

0.00 IN A COLUMN INDICATES A TOTAL OF $<0.005$ 


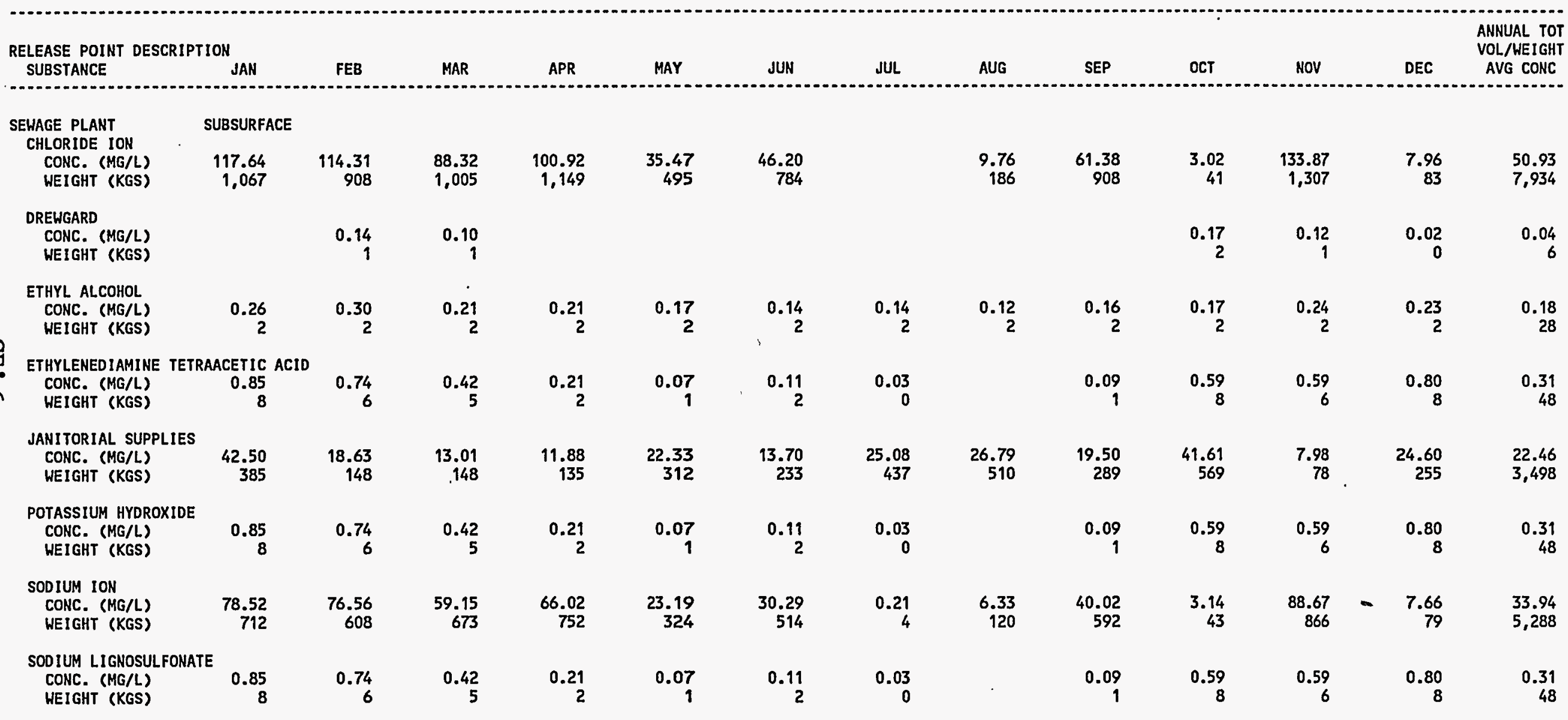


PAGE: 3

SCHED. NO. INRPTOO8
IDAHO OPERATIONS OFFICE

UNITED STATES DEPARTMENT OF ENERGY

12-JUL-95

INEL NONRADIOLOGICAL HASTE MANAGEMENT INFORMATION SYSTEM

FOR JANUARY THROUGH DECEMBER 1994

CFA RELEASE POINT AND LIQUID DISPOSED SUBSTANCES SUMMARY

RPT 135-I

CFA

\begin{tabular}{|c|c|c|c|c|c|c|c|c|c|c|c|c|c|}
\hline $\begin{array}{l}\text { RELEASE POINT DES } \\
\text { SUBSTANCE }\end{array}$ & ${ }_{\text {JION }}^{\text {JAN }}$ & FEB & MAR & APR & MAY & JUN & JUL & AUG & SEP & OCT & NOV & DEC & $\begin{array}{c}\text { ANNUAL TOT } \\
\text { VOL/WEIGHT } \\
\text { AVG CONC }\end{array}$ \\
\hline $\begin{array}{l}\text { SEWAGE PLANT } \\
\text { SULFITE ION }\end{array}$ & SUBSURF & & & & & & & & & & & & \\
\hline $\begin{array}{l}\text { CONC. (MG/L) } \\
\text { WEIGHT (KGS) }\end{array}$ & $\begin{array}{r}3.88 \\
35\end{array}$ & $\begin{array}{r}3.82 \\
30\end{array}$ & $\begin{array}{r}3.23 \\
37\end{array}$ & $\begin{array}{r}1.10 \\
13\end{array}$ & $\begin{array}{r}0.33 \\
5\end{array}$ & $\begin{array}{r}0.55 \\
9\end{array}$ & $\begin{array}{r}0.37 \\
6\end{array}$ & & $\begin{array}{r}0.42 \\
6\end{array}$ & $\begin{array}{r}2.04 \\
28\end{array}$ & $\begin{array}{r}3.28 \\
32\end{array}$ & $\begin{array}{r}4.01 \\
42\end{array}$ & $\begin{array}{r}1.56 \\
243\end{array}$ \\
\hline
\end{tabular}

㔖

DETAILS MAY NOT ADD UP TO TOTALS BECAUSE OF ROUNDING

0 IN A COLUMN INDICATES A TOTAL OF $<0.5$

0.00 IN A COLUMN INDICATES A TOTAL OF $<0.005$ 
PAGE: 1

SCHED. NO. INRPTOO9
IOAHO OPERATIONS OFFICE

$11-J U L-95$

UNITED STATES DEPARTMENT OF ENERGY

INEL NONRADIOLOGICAL WASTE MANAGEMENT INFORMATION SYSTEM

FOR JANUARY THROUGH DECEMBER 1994

CFA LANDFILL SOLID DISPOSED HASTE SUMMARY

RPT 136-1

CFA

(ALL VOLUMES = CUBIC METERS)

\begin{tabular}{|c|c|c|c|c|c|c|c|c|c|c|c|c|c|}
\hline Type of Haste & JAN & FEB & MAR & APR & MAY & JUN & JUL & AUG & SEP & OCT & NOV & DEC & TOTALS \\
\hline $\begin{array}{l}\text { ASBESTOS } \\
\text { ASPHALT } \\
\text { CAFETERIA GARBAGE } \\
\text { DIRT AND GRAVEL. } \\
\text { MASONRY AND CONCRETE } \\
\text { SCRAP METAL } \\
\text { TRASH, SWEEPINGS, ETC. } \\
\text { WEEDS, GRASS AND TREES } \\
\text { WOOD AND SCRAP LUMBER }\end{array}$ & $\begin{array}{r}3.8 \\
165.9 \\
18.4 \\
78.0 \\
6.5 \\
502.7 \\
28.3\end{array}$ & $\begin{array}{r}163.6 \\
18.7 \\
41.3 \\
483.6 \\
18.4\end{array}$ & $\begin{array}{r}144.5 \\
87.9 \\
64.2 \\
20.6 \\
680.5 \\
3.1 \\
21.8\end{array}$ & $\begin{array}{r}9.9 \\
0.8 \\
136.5 \\
12.6 \\
24.8 \\
32.9 \\
446.1 \\
1.1 \\
21.4\end{array}$ & $\begin{array}{r}5.7 \\
171.3 \\
135.0 \\
566.6 \\
24.5 \\
490.5 \\
0.4 \\
18.4\end{array}$ & $\begin{array}{r}0.8 \\
195.7 \\
176.6 \\
29.4 \\
34.0 \\
510.4 \\
0.8 \\
35.6\end{array}$ & $\begin{array}{r}68.0 \\
160.6 \\
7.3 \\
460.7 \\
40.1 \\
520.7 \\
\\
26.0\end{array}$ & $\begin{array}{r}166.7 \\
69.6 \\
45.9 \\
57.0 \\
676.7 \\
19.1 \\
36.3\end{array}$ & $\begin{array}{r}6.9 \\
188.5 \\
12.6 \\
682.0 \\
25.6 \\
537.5 \\
14.1 \\
52.8\end{array}$ & $\begin{array}{r}162.1 \\
8.0 \\
691.6 \\
59.3 \\
577.3 \\
11.5 \\
26.4\end{array}$ & $\begin{array}{r}124.6 \\
39.8 \\
174.7 \\
71.9 \\
57.3 \\
39.0 \\
579.2 \\
3.1 \\
78.8\end{array}$ & $\begin{array}{r}6.9 \\
134.6 \\
514.6 \\
13.8 \\
23.7 \\
423.2 \\
13.8\end{array}$ & $\begin{array}{r}146.8 \\
132.7 \\
1.964 .6 \\
1.114 .4 \\
2,733.1 \\
404.5 \\
6,428.4 \\
53.1 \\
377.7\end{array}$ \\
\hline GRAND TOTALS: & 803.6 & 725.6 & $1,022.7$ & 686.2 & $1,412.2$ & 983.3 & $1,283.4$ & $1,083.4$ & $1,520.0$ & $1,536.1$ & $1,168.3$ & $1,130.5$ & $13,355.3$ \\
\hline
\end{tabular}


PAGE: 1

SCHED. NO. INRPTO1O

FOR JANUARY THROUGH DECEMBER 1994

CFA WATER USAGE AND DISPOSAL SUMMARY

RPT 137-1

(ALL VOLUMES = NEAREST THOUSAND LITERS)

CFA

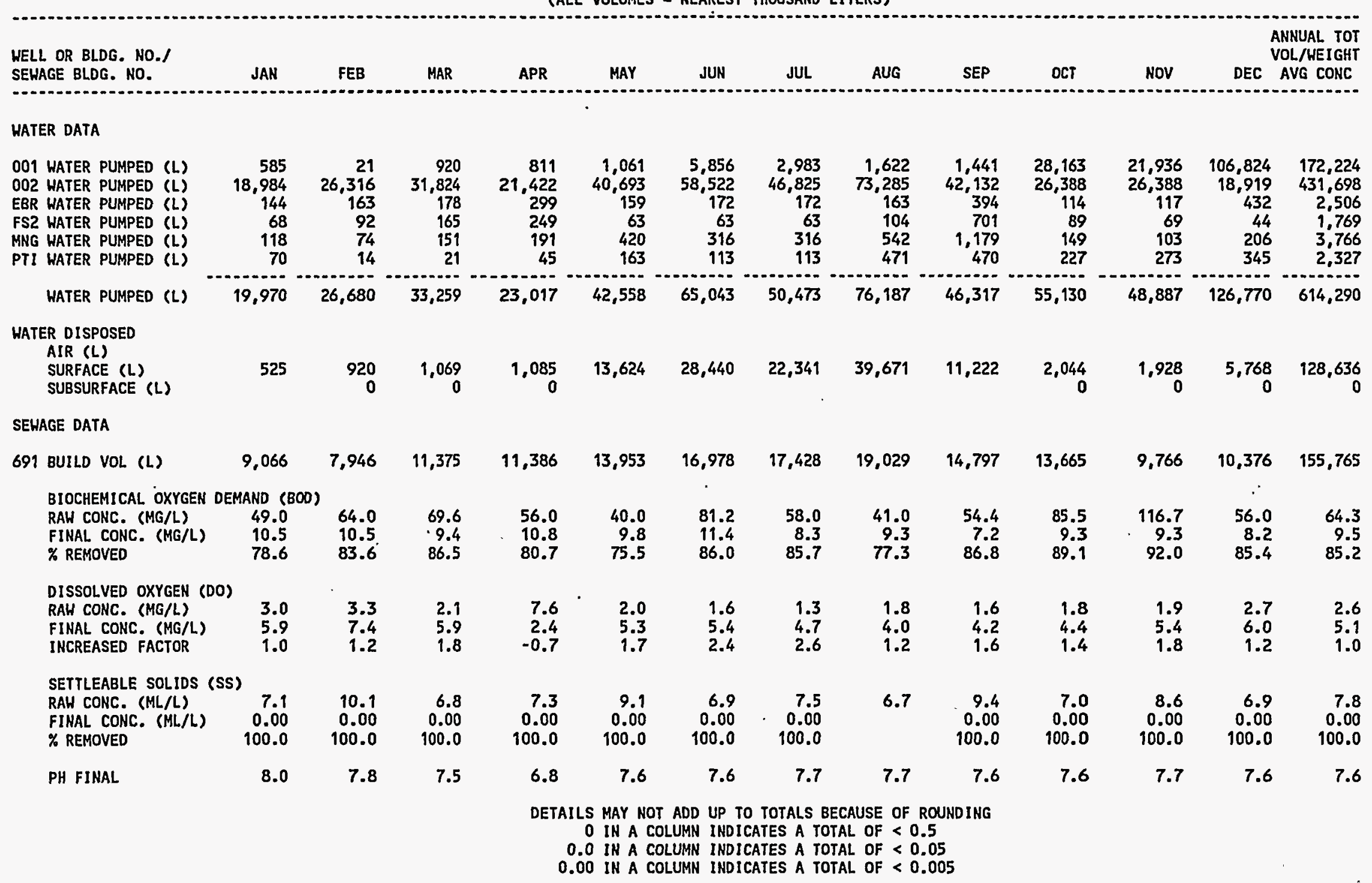


PAGE: 2

SCHED. NO. INRPTO10
IDAHO OPERATIONS OFFICE

UNITED STATES DEPARTMENT OF ENERGY

INEL NONRADIOLOGICAL HASTE MANAGEMENT INFORMATION SYSTEM

FOR JANUARY THROUGH DECEMBER 1994

CFA WATER USAGE AND DISPOSAL SUMMARY

RPT $137-1$

CFA

(ALL VOLUMES = NEAREST THOUSAND LITERS)

ANNUAL TOT

VOL/WEIGHT

HELL OR BLDG. NO./

SEWAGE BLDG. NO.

JAN

FEB MAR APR

MAY

JUN

JUL

AUG

SEP

OCT

NoV

DEC AVG CONC

\section{TOTAL SEWAGE(L)}

9,066

7,946

11,375

11,386

13,953

$16,978 \quad 17,428$

19,029

14,797

13,665

9,766

10,376

155,765

TOTAL HATER DISPOSED(L) $\quad 9,591$

8,865

12,444

12,471

27,577

45,417

39,769

58,700

26,019

15,710

11,694

16,144

284,401

ACCOUNTABBILITY (\%)

48.0

33.2

37.4

54.2

$64.8 \quad 69.8$

78.8

77.0

56.2

28.5

23.9

12.7

46.3 
PAGE: 1

SCHED. NO. INRPTO11
IDAHO OPERATIONS OFFICE

13-JUL-95

UNITED STATES DEPARTMENT OF ENERGY

INEL NONRADIOLOGICAL WASTE MANAGEMENT INFORMATION SYSTEH

FOR JANUARY THROUGH DECEMBER 1994

CFA FUEL OIL USAGE AND STACK EFFLUENTS SUMMARY

RPT 138-1

CFA

(ALL VALUES $=$ NEAREST THOUSAND)

(ALL. VALUES = NEAREST THOUSAND)
FUEL OIL TYPE

ANHUAL

JAN

FEB

APR

MAY

JUN

JUL.

AUG

SEP

OCT

Nov

DEC

TYPE 2

VOLUME (LITERS)

SO2 (KGS)

NOX (KGS)

PARTICULATES (KGS)

DIESEL.

VOLUME (LITERS)

SO2 (KGS)

PARTICULATES (KGS)

GRAND TOTALS:

VOLUME (LITERS)

SO2 (KGS)

NOX (KGS)

PARTICULATES (KGS)

$\begin{array}{rrr}67 & 60 & 28 \\ 0 & 0 & 0\end{array}$

37
0

18
0

$\begin{array}{ll}9 & 9 \\ 0 & 0\end{array}$

9

14
0

18

18
0

13
0

21
0

104
1

398

$\begin{array}{rrrr}28 & 7 & 2 & 2 \\ 0 & 0 & 0 & 0\end{array}$

$\begin{array}{ll}8 & 3 \\ 0 & 0\end{array}$

7
0

9

9
0

14
0

5
0

93
1

$94 \quad 68$

30
0

38
0

$\begin{array}{rrr}18 & 17 & 12 \\ 0 & 0 & 0\end{array}$

DETAILS MAY NOT ADD UP TO TOTALS BECAUSE OF ROUNDING

0 IN A COLUMN INDICATES A TOTAL OF $<0.5$ 


\section{Chemical Processing Plant 1994 Detail Data}

Report 144-CPP Airborne Disposed Substances Summary . . . . . . . . . . CPP-3

Report 144-CPP Airborne Release Point And Disposed Substances Summary .. CPP-4

Report 145-CPP Liquid Disposed Substances Summary . . . . . . . . . . C CPP-5

Report 145-CPP Release Point And Liquid Disposed Substances Summary . . . CPP-6

Report 146-CPP Landfill Solid Disposed Waste Summary . . . . . . . . . . . . . CPP-8

Report 147-CPP Water Usage And Disposal Summary . . . . . . . . . . . . . . CPP-9

Report 148-CPP Fuel Oil Usage And Stack Effluents Summary . . . . . . . . . . CPP-11

Report 8-INEL Coal Summary For January Through December 1994 . . . . . . CPP-12 
PAGE: 1

SCHED. NO. INRPTOOTA

IDAHO OPERATIONS OFFICE

13-JUL-95

UNITED STATES DEPARTMENT OF ENERGY

INEL NONRADIOLOGICAL HASTE MANAGEMENT INFORMATION SYSTEM

FOR JANUARY THROUGH DECEMBER 1994

CPP AIRBORNE DISPOSED SUBSTANCES SUMMARY

RPT 144-I

CPP

\begin{tabular}{|c|c|c|c|c|c|c|c|c|c|c|c|c|c|}
\hline SUBSTANCE & JAN & FEB & MAR & APR & MAY & JUN & JUL & AUG & SEP & OCT & NOV & DEC & $\begin{array}{l}\text { ANNUAL } \\
\text { TOTALS }\end{array}$ \\
\hline $\begin{array}{l}\text { NITRIC OXIDE } \\
\text { WEIGHT (KGS) }\end{array}$ & 0 & 0 & 0 & 0 & 0 & 0 & 0 & 0 & 0 & 0 & 0 & 0 & 0 \\
\hline $\begin{array}{l}\text { NITROGEN DIOXIDE } \\
\text { WEIGHT (KGS) }\end{array}$ & 0 & 0 & 0 & 0 & 0 & 0 & 0 & 0 & 0 & 0 & 0 & 0 & 0 \\
\hline
\end{tabular}

荢

$O$ IN A COLUMN INDICATES A TOTAL OF $<0.5$ 
PAGE: 1

SCHED. NO. INRPTOO7

IDAHO OPERATIONS OFFICE

13-JUL-95

UNITED STATES DEPARTMENT OF ENERGY

INEL NONRADIOLOGICAL WASTE MANAGEMENT INFORMATION SYSTEM

FOR JANUARY THROUGH DECEMBER 1994

CPP AIRBORNE RELEASE POINT AND DISPOSED SUBSTANCES SUMMARY

RPT 144-I

CPP

ANNUAL TOT

VOL/WEIGHT

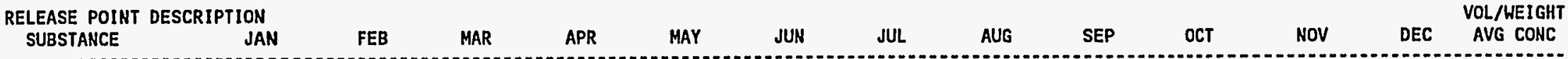

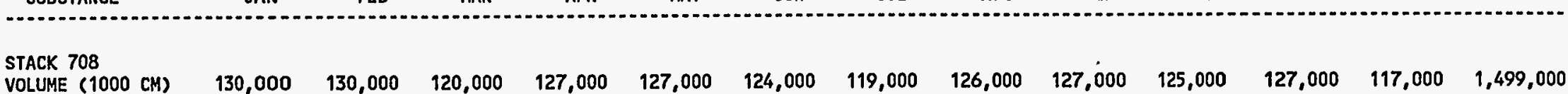

VOLUME (1000 CM)

130,000

$130,000-120,000$

$(000$

NITRIC OXIOE

CONC. (CC/CM)

$0.00 \quad 0.00$

0.00

$0.00 \quad 0.00$

0.00

0.00

0.00

0.00

0.00

0.00

0.00

0.00
0

NITROGEN DIOXIDE CONC. (CC/CM)

$0.00 \quad 0.00$

0.00

0.00

0.00

0.00

0.00

0.00

0.00

0.00

0.00

0.00
0

0.00 
CPP LIQUID DISPOSED SUBSTANCES SUMMARY

RPT 145-1

\begin{tabular}{|c|c|c|c|c|c|c|c|c|c|c|c|c|c|}
\hline SUBSTANCE & JAN & FEB & MAR & APR & MAY & JUN & JUL & AUG & SEP & OCT & HOV & DEC & $\begin{array}{l}\text { ANNUAL } \\
\text { TOTALS }\end{array}$ \\
\hline $\begin{array}{l}\text { ARSENIC } \\
\text { WEIGHT (KGS) }\end{array}$ & & 0 & & & & & & & & & & & 0 \\
\hline $\begin{array}{l}\text { BARIUM } \\
\text { HEIGHT (KGS) }\end{array}$ & 21 & 18 & 15 & 18 & 18 & 16 & 0 & 4 & 14 & 15 & 15 & 15 & 169 \\
\hline $\begin{array}{l}\text { CADMIUM } \\
\text { HEIGHT (KGS) }\end{array}$ & 2 & & & & & & & & & & & & 2 \\
\hline $\begin{array}{l}\text { CHLORIDE ION } \\
\text { WEIGHT (KGS) }\end{array}$ & 56,934 & 40,928 & 56,734 & 53,323 & 46,087 & 48,605 & 0 & 14,670 & 44,282 & 38,970 & 40,529 & 51,063 & 492,125 \\
\hline $\begin{array}{l}\text { CHROMIUM } \\
\text { HEIGHT (KGS) }\end{array}$ & & & & & 3 & 2 & & & & & 2 & & 7 \\
\hline $\begin{array}{l}\text { FLUORIDE ION } \\
\text { WEIGHT (KGS) }\end{array}$ & & 35 & 46 & 42 & 44 & 31 & .0 & 9 & 35 & 30 & 33 & 32 & 335 \\
\hline $\begin{array}{l}\text { LEAD } \\
\text { WEIGHT (KGS) }\end{array}$ & & & & & & & & & & & & 5 & 5 \\
\hline $\begin{array}{l}\text { NITRATE ION } \\
\text { WEIGHT (KGS) }\end{array}$ & 1,095 & 737 & 1,008 & 1,250 & 2,231 & 2,665 & 0 & 225 & 784 & 696 & 771 & 737 & 12,198 \\
\hline $\begin{array}{l}\text { SILVER } \\
\text { WEIGHT (KGS) }\end{array}$ & & & & & & 4 & & & & & & & 4 \\
\hline $\begin{array}{l}\text { SODIUM ION } \\
\text { WEIGHT (KGS) }\end{array}$ & 39,917 & 24,711 & 33,251 & 33,796 & 31,339 & 27,255 & 0 & 2,378 & 28,913 & 21,179 & 25,198 & 25,162 & 293,099 \\
\hline $\begin{array}{l}\text { SULFATE ION } \\
\text { WEIGHT (KGS) }\end{array}$ & 5,777 & 4,541 & 5,944 & 5,257 & 4,996 & 4,330 & 0 & 1,108 & 4,413 & 3,911 & 4,357 & 4,070 & 48,704 \\
\hline
\end{tabular}




\begin{tabular}{|c|c|c|c|c|c|c|c|c|c|c|c|c|c|}
\hline $\begin{array}{l}\text { RELEASE POINT DESCR } \\
\text { SUBSTANCE }\end{array}$ & TION JAN & FEB & MAR & APR & MAY & JUN & JUL & AUG & SEP & OCT & Nov & DEC & $\begin{array}{l}\text { ANNUAL TOT } \\
\text { VOL/WEIGHT } \\
\text { AVG CONC }\end{array}$ \\
\hline $\begin{array}{l}\text { S PERCOLATION POND } \\
\text { VOLUME }(1000 \mathrm{~L}) \\
\text { PH }\end{array}$ & $\begin{array}{l}\text { SURFACE } \\
210,090\end{array}$ & 154,444 & 207,818 & 187,756 & 184,349 & 151,416 & 133,246 & 37,800 & 152,173 & 141,195 & 151,795 & 148,009 & $1,860,092$ \\
\hline $\begin{array}{l}\text { ARSENIC } \\
\text { CONC. (MG/L) } \\
\text { WEIGHT (KGS) }\end{array}$ & & $\begin{array}{r}0.00 \\
0\end{array}$ & & & & & & & & & & & $\begin{array}{r}0.00 \\
0\end{array}$ \\
\hline $\begin{array}{l}\text { BARIUM } \\
\text { CONC. (MG/L) } \\
\text { HEIGHT (KGS) }\end{array}$ & $\begin{array}{r}0.10 \\
21\end{array}$ & $\begin{array}{r}0.12 \\
18\end{array}$ & $\begin{array}{r}0.07 \\
15\end{array}$ & $\begin{array}{r}0.09 \\
18\end{array}$ & $\begin{array}{r}0.10 \\
18\end{array}$ & $\begin{array}{r}0.11 \\
16\end{array}$ & $\begin{array}{r}0.09 \\
12\end{array}$ & $\begin{array}{r}0.10 \\
4\end{array}$ & $\begin{array}{r}0.09 \\
14\end{array}$ & $\begin{array}{r}0.11 \\
15\end{array}$ & $\begin{array}{r}0.10 \\
15\end{array}$ & $\begin{array}{r}0.10 \\
15\end{array}$ & $\begin{array}{r}0.10 \\
181\end{array}$ \\
\hline $\begin{array}{l}\text { CADMIUM } \\
\text { CONC. (MG/L) } \\
\text { HEIGHT (KGS) }\end{array}$ & $\begin{array}{r}0.01 \\
2\end{array}$ & & & & & & & & & & & & $\begin{array}{r}0.00 \\
2\end{array}$ \\
\hline $\begin{array}{l}\text { CHLORIDE ION } \\
\text { CONC. (MG/L) } \\
\text { HEIGHT (KGS) }\end{array}$ & $\begin{array}{l}271.00 \\
56,934\end{array}$ & $\begin{array}{l}265.00 \\
40,928\end{array}$ & $\begin{array}{l}273.00 \\
56,734\end{array}$ & $\begin{array}{l}284.00 \\
53,323\end{array}$ & $\begin{array}{l}250.00 \\
46,087\end{array}$ & $\begin{array}{l}321.00 \\
48,605\end{array}$ & $\begin{array}{l}271.00 \\
36,110\end{array}$ & $\begin{array}{l}388.09 \\
14,670\end{array}$ & $\begin{array}{l}291.00 \\
44,282\end{array}$ & $\begin{array}{l}276.00 \\
38,970\end{array}$ & $\begin{array}{l}267.00 \\
40,529\end{array}$ & $\begin{array}{l}345.00 \\
51,063\end{array}$ & $\begin{array}{r}283.98 \\
528,235\end{array}$ \\
\hline $\begin{array}{l}\text { CHROMIUM } \\
\text { CONC. (MG/L) } \\
\text { HEIGHT (KGS) }\end{array}$ & & - & & & $\begin{array}{r}0.02 \\
3\end{array}$ & $\begin{array}{r}0.01 \\
2\end{array}$ & & & . & & $\begin{array}{r}0.01 \\
2\end{array}$ & & $\begin{array}{r}0.00 \\
7\end{array}$ \\
\hline $\begin{array}{l}\text { FLUORIDE ION } \\
\text { CONC. (MG/L) } \\
\text { HEIGHT (KGS) }\end{array}$ & & $\begin{array}{r}0.23 \\
35\end{array}$ & $\begin{array}{r}0.22 \\
46\end{array}$ & $\begin{array}{r}0.22 \\
42\end{array}$ & $\begin{array}{r}0.24 \\
44\end{array}$ & $\begin{array}{r}0.21 \\
31\end{array}$ & $\begin{array}{r}0.22 \\
29\end{array}$ & $\begin{array}{r}0.23 \\
9\end{array}$ & $\begin{array}{r}0.23 \\
35\end{array}$ & $\begin{array}{r}0.21 \\
30\end{array}$ & $\begin{array}{r}0.22 \\
33\end{array}$ & $\begin{array}{r}0.21 \\
32\end{array}$ & $\begin{array}{r}0.20 \\
364\end{array}$ \\
\hline $\begin{array}{l}\text { LEAD } \\
\text { CONC. (MG/L) } \\
\text { HEIGHT (KGS) }\end{array}$ & & & & & & & & & & & & $\begin{array}{r}0.03 \\
5\end{array}$ & $\begin{array}{r}0.00 \\
5\end{array}$ \\
\hline $\begin{array}{l}\text { NITRATE ION } \\
\text { CONC. (MG/L) } \\
\text { WEIGHT (KGS) }\end{array}$ & $\begin{array}{r}5.21 \\
1,095\end{array}$ & $\begin{array}{r}4.77 \\
737\end{array}$ & $\begin{array}{r}4.85 \\
1,008\end{array}$ & $\begin{array}{r}6.66 \\
1,250\end{array}$ & $\begin{array}{l}12.10 \\
2,231\end{array}$ & $\begin{array}{l}17.60 \\
2,665\end{array}$ & $\begin{array}{r}5.45 \\
726\end{array}$ & $\begin{array}{r}5.95 \\
225\end{array}$ & $\begin{array}{r}5.15 \\
784\end{array}$ & $\begin{array}{r}4.93 \\
696\end{array}$ & $\begin{array}{r}5.08 \\
771\end{array}$ & $\begin{array}{r}4.98 \\
737\end{array}$ & $\begin{array}{r}6.95 \\
12,924\end{array}$ \\
\hline
\end{tabular}




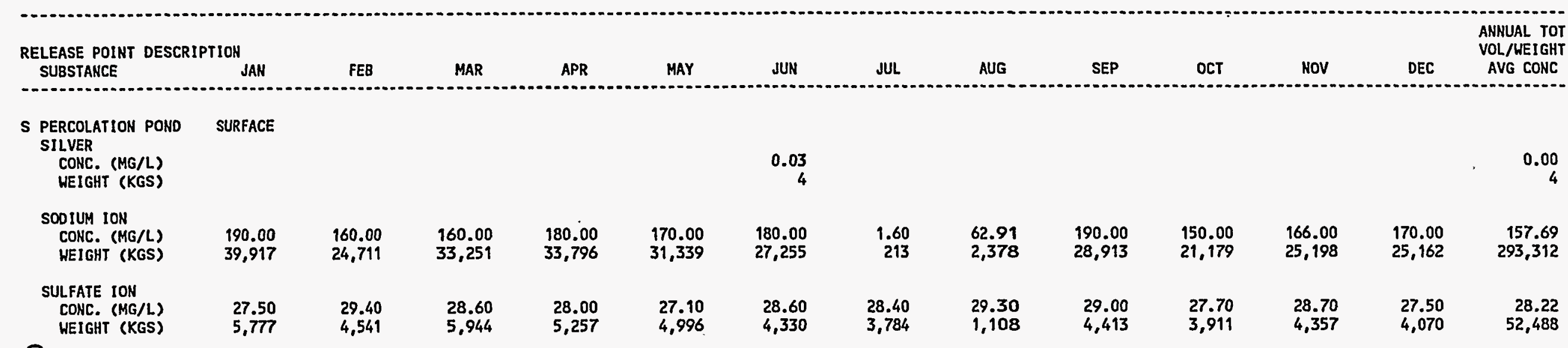


PAGE: 1

SCHED. NO. INRPTOOS

IDAHO OPERATIONS OFFICE

UNITED STATES DEPARTMENT OF ENERGY

INEL. NONRADIOLOGICAL WASTE MAMAGEMENT INFORMATION SYSTEM

FOR JANUARY THROUGH DECEMBER 1994

CPP LANDFILL SOLID DISPOSED HASTE SUMMARY

RPT $146-I$
CPP

(ALL VOLUMES $=$ CUBIC METERS)

\begin{tabular}{|c|c|c|c|c|c|c|c|c|c|c|c|c|c|}
\hline Type of Waste & JAN & FEB & MAR & APR & MAY & JUN & JUL & AUG & SEP & OCT & NOV & DEC & TOTALS \\
\hline $\begin{array}{l}\text { ASBESTOS } \\
\text { ASPHALT' } \\
\text { CAFETERIA GARBAGE } \\
\text { DIRT AND GRAVEL. } \\
\text { MASONRY AND CONCRETE } \\
\text { SCRAP METAL. } \\
\text { TRASH, SWEEPINGS, ETC. } \\
\text { WEEDS, GRASS AND TREES } \\
\text { WOOD AND SCRAP LUMBER }\end{array}$ & $\begin{array}{r}3.4 \\
18.4 \\
110.9 \\
3.1 \\
3.1 \\
626.2 \\
17.2\end{array}$ & $\begin{array}{r}223.3 \\
1.5 \\
6.5 \\
496.6 \\
41.3\end{array}$ & $\begin{array}{r}45.9 \\
5,500.5 \\
76.1 \\
27.9 \\
597.5 \\
87.5\end{array}$ & $\begin{array}{r}144.5 \\
5.654 .2 \\
58.1 \\
9.9 \\
516.5 \\
6.1 \\
65.0\end{array}$ & $\begin{array}{r}107.0 \\
485.5 \\
1,292.9 \\
20.3 \\
34.0 \\
521.8 \\
1.9 \\
31.7\end{array}$ & $\begin{array}{r}195.4 \\
410.6 \\
107.8 \\
26.8 \\
1,148.0 \\
37.8\end{array}$ & $\begin{array}{r}96.3 \\
123.1 \\
3,119.6 \\
10.3 \\
21.0 \\
682.8 \\
27.9\end{array}$ & $\begin{array}{r}644.9 \\
12.2 \\
2,952.1 \\
19.9 \\
113.2 \\
576.5 \\
0.4 \\
148.0\end{array}$ & $\begin{array}{r}393.8 \\
1,608.0 \\
105.1 \\
45.5 \\
458.4 \\
1.9 \\
59.3\end{array}$ & $\begin{array}{r}1,288.4 \\
1,261.2 \\
95.2 \\
37.1 \\
516.1 \\
6.1 \\
34.4\end{array}$ & $\begin{array}{r}118.5 \\
1.139 .3 \\
211.8 \\
49.3 \\
453.4 \\
37.5\end{array}$ & $\begin{array}{r}6.1 \\
182.0\end{array}$ & $\begin{array}{r}203.4 \\
3,443.8 \\
30.6 \\
23,272.5 \\
709.2 \\
380.4 \\
6,775.9 \\
16.4 \\
587.6\end{array}$ \\
\hline GRAND TOTALS: & 782.2 & 769.2 & $6,335.5$ & $6,454.4$ & $2,495.3$ & $1,926.4$ & $4,081.1$ & $4,467.2$ & $2,671.9$ & $3,238.5$ & $2,009.8$ & 188.5 & $35,419.7$ \\
\hline
\end{tabular}


FOR JANUARY THROUGH DECEMBER 1994

CPP WATER USAGE AND DISPOSAL SUMMARY

CPP

(ALL. VOLUMES = NEAREST THOUSAND LITERS)

ANNUAL TOT

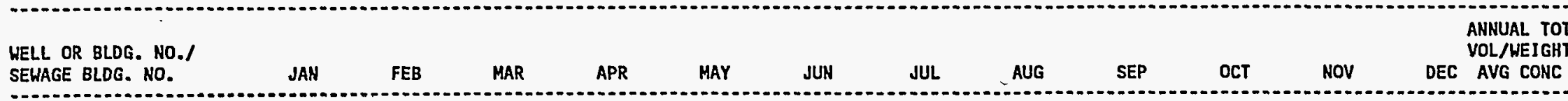

SEWAGE BLDG NO.

\section{WATER DATA}

616 WATER PUMPED (L)

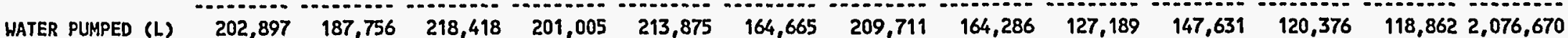

WATER DISPOSED

\begin{tabular}{|c|c|c|c|c|c|c|c|c|c|c|c|c|}
\hline $\begin{array}{l}\text { SURFACE (L) } \\
\text { SUBSURFACE (L) }\end{array}$ & 210,090 & 154.444 & 207,818 & 187,756 & 184,349 & 151,416 & 133,246 & 143,088 & 152,552 & 141,195 & 151,795 & $148,009 \quad 1,965,758$ \\
\hline
\end{tabular}

SEWAGE DATA

615 BUILD VOL

BIOCHEMICAL OXYGEN DEMAND (BOD)

RAW CONC. (MG/L) 155.0

FINAL CONC. (MG/L) $\quad 15.0$

$\%$ REMOVED

DISSOLVED OXYGEN (DO)

RAH CONC. (MG/L)

FINAL CONC. ( $M G / L)$
INCREASED FACTOR

$1.1 \quad 0.9$

SETTLEABLE SOLIDS (SS)

RAW CONC. (HL/L)

FINAL CONC. (ML/L)

\% REMOVED

$\begin{array}{lll}\text { PH FINAL } & 8.0 & 7.8\end{array}$

TOTAL SEWAGE(L) $\quad 11,773 \quad 5,186$

\begin{tabular}{|c|c|c|c|c|c|c|c|c|c|}
\hline 7,230 & 16,807 & 22,372 & 11,924 & 7,382 & 5,413 & 3,861 & 6,208 & 7,533 & 105,688 \\
\hline $\begin{array}{r}158.0 \\
33.0 \\
79.1\end{array}$ & $\begin{array}{r}100.0 \\
37.0 \\
63.0\end{array}$ & $\begin{array}{r}164.0 \\
57.0 \\
65.2\end{array}$ & $\begin{array}{r}123.0 \\
47.0 \\
61.8\end{array}$ & $\begin{array}{r}103.0 \\
17.0 \\
83.5\end{array}$ & $\begin{array}{l}92.0 \\
16.0 \\
82.6\end{array}$ & $\begin{array}{r}133.0 \\
22.0 \\
83.5\end{array}$ & $\begin{array}{l}90.0 \\
10.0 \\
88.9\end{array}$ & $\begin{array}{l}98.0 \\
10.0 \\
89.8\end{array}$ & $\begin{array}{r}120.4 \\
26.0 \\
78.4\end{array}$ \\
\hline $\begin{array}{l}0.9 \\
6.9 \\
6.7\end{array}$ & $\begin{array}{l}1.1 \\
5.5 \\
4.0\end{array}$ & $\begin{array}{l}1.1 \\
6.4 \\
4.8\end{array}$ & $\begin{array}{l}0.9 \\
5.0 \\
4.6\end{array}$ & $\begin{array}{l}0.8 \\
3.5 \\
3.4\end{array}$ & $\begin{array}{l}0.8 \\
4.9 \\
5.1\end{array}$ & $\begin{array}{l}1.2 \\
7.2 \\
5.0\end{array}$ & $\begin{array}{l}1.7 \\
9.3 \\
4.5\end{array}$ & $\begin{array}{r}1.5 \\
10.3 \\
5.9\end{array}$ & $\begin{array}{l}1.1 \\
6.1 \\
4.6\end{array}$ \\
\hline
\end{tabular}

TOTAL. WATER DISPOSED(L) 221,862 159,630

$\begin{array}{rrrrrrrrrrr} & 7.9 & 8.0 & 8.4 & 9.0 & 7.7 & 7.7 & 8.0 & 8.4 & 8.1 & 8.1 \\ & 7,230 & 16,807 & 22,372 & 11,924 & 7,382 & 5,413 & 3,861 & 6,208 & 7,533 & 105,688 \\ 207,818 & 194,986 & 201,156 & 173,788 & 145,170 & 150,470 & 157,965 & 145,057 & 158,003 & 155,542 & 2,071,447\end{array}$

DETAILS MAY NOT ADD UP TO TOTALS BECAUSE OF 'ROUNDING

0 IN A COLUMN INDICATES A TOTAL OF $<0.5$

0.0 IN A COLUMN INDICATES A TOTAL OF $<0.05$

0.00 IH A COLUMN INDICATES A TOTAL OF $<0.005$ 
PAGE: 2

SCHED. NO. INRPTO1O
IDAHO OPERATIONS OFFICE

UNITED STATES DEPARTMENT OF ENERGY

INEL NONRADIOLOGICAL WASTE MANAGEMENT INFORMATION SYSTEM

FOR JANUARY THROUGH DECEMBER 1994

CPP WATER USAGE AND DISPOSAL. SUMMARY

(ALL VOLUMES $=$ NEAREST THOUSAND LITERS)

CPP

ANNUAL TOT

ANNUAL TOT
VOL/WEIGHT

HELL OR BLDG. NO.

SEWAGE BLDG. NO.

JAN

ACCOUNTABILITY $(\%)$

109.3

91.6

98.3

130.9

99.7 
PAGE: 1

IDAHO OPERATIONS OFFICE

13-JUL-95

SCHED. NO. INRPTO11

UNITED STATES DEPARTMENT OF ENERGY

IHEL NONRADIOLOGICAL WASTE MANAGEMENT INFORMATION SYSTEM

FOR JANUARY THROUGH DECEMBER 1994

CPP FUEL OIL USAGE AND STACK EFFLUENTS SUMMARY

RPT 148-I

(ALL. VALUES $=$ MEAREST THOUSAND)

\begin{tabular}{|c|c|c|c|c|c|c|c|c|c|c|c|c|c|}
\hline $\begin{array}{l}\text { FUEL OIL TYPE } \\
\text { EFFLUENTS }\end{array}$ & JAN & FEB & MAR & APR & MAY & JUN & JUL & AUG & SEP & OCT & NOV & DEC & $\begin{array}{l}\text { ANNUAL } \\
\text { TOTALS }\end{array}$ \\
\hline $\begin{array}{l}\text { TYPE } 2 \\
\text { VOLUME (LITERS) } \\
\text { SOL (KGS) } \\
\text { NOX (KGS) } \\
\text { PARTICULATES (KGS) }\end{array}$ & $\begin{array}{r}277 \\
5\end{array}$ & $\begin{array}{r}306 \\
5\end{array}$ & $\begin{array}{r}893 \\
15\end{array}$ & $\begin{array}{r}821 \\
14\end{array}$ & $\begin{array}{r}488 \\
8\end{array}$ & $\begin{array}{r}186 \\
3\end{array}$ & $\begin{array}{r}18 \\
0\end{array}$ & $\begin{array}{l}0 \\
0\end{array}$ & $\begin{array}{r}210 \\
3\end{array}$ & $\begin{array}{r}613 \\
10\end{array}$ & $\begin{array}{r}329 \\
5\end{array}$ & $\begin{array}{r}110 \\
2\end{array}$ & $\begin{array}{r}4,252 \\
71\end{array}$ \\
\hline $\begin{array}{l}\text { GRAND TOTALS: } \\
\text { VOLUME (LITERS) } \\
\text { SO2 (KGS) } \\
\text { HOX (KGS) } \\
\text { PART ICULATES (KGS) }\end{array}$ & $\begin{array}{r}277 \\
5\end{array}$ & $\begin{array}{r}306 \\
5\end{array}$ & $\begin{array}{r}893 \\
15\end{array}$ & $\begin{array}{r}821 \\
14\end{array}$ & $\begin{array}{r}488 \\
8\end{array}$ & $\begin{array}{r}186 \\
3\end{array}$ & $\begin{array}{r}18 \\
0\end{array}$ & $\begin{array}{l}0 \\
0\end{array}$ & $\begin{array}{r}210 \\
3\end{array}$ & $\begin{array}{r}613 \\
10\end{array}$ & $\begin{array}{r}329 \\
5\end{array}$ & $\begin{array}{r}110 \\
2\end{array}$ & $\begin{array}{r}4,252 \\
71\end{array}$ \\
\hline
\end{tabular}

芯

DETAILS MAY NOT ADD UP TO TOTALS BECAUSE OF ROUNDING

0 IN A COLUMN INDICATES A TOTAL OF $<0.5$ 
PAGE: 1

SCHED. NO. INRPTO2O
IDAHO OPERATIONS OFFICE

UNITED STATES DEPARTMENT OF ENERGY

13-JUL-95

INEL COAL SUMMARY

RPT 8-1

FOR JANUARY THROUGH DECEMBER 1994

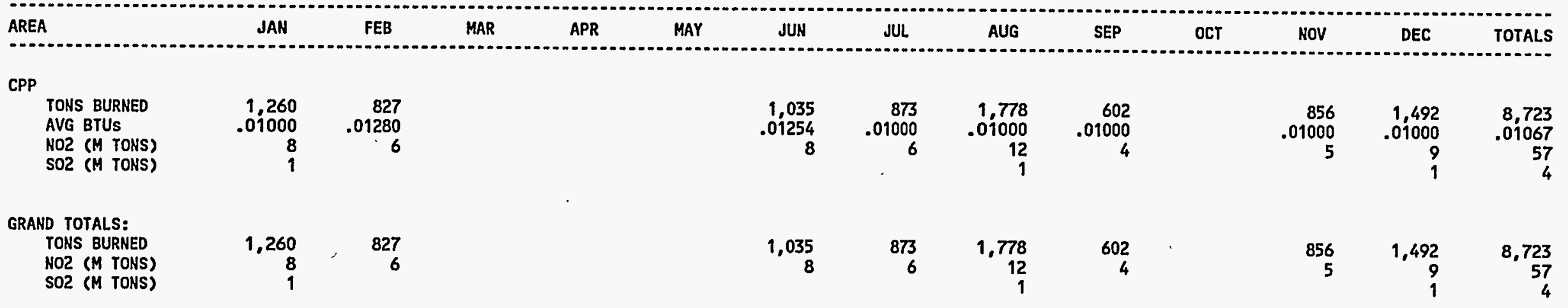

雚

BASED ON AN EXTENSIVE REVIEH OF EMISSIONS DATA, CORRECTTIONS TO SO2 EMITIED DATA HAVE BEEN PPERFORMED. DETAILS MAY NOT ADD UP TO TOTALS BECAUSE OF ROUMDING

0 IN A COLUMN INDICATES A TOTAL OF $<0.5$ 
Decontamination and Decommissioning 1994 Detail Data

Report 6-DAD Landfill Solid Disposed Waste Summary ............ D\&D-3 . 
PAGE: 1

IDAHO OPERATIONS DFFICE

24-MAR-95

SCHED. NO. IHRPTOOO

UHITED STATES DEPARTMENT OF ENERGY

IOLOGICAL UASTE MANAGEMENT IHFORMATIOH SYSTEM

FOR JANUARY THROUGH DECEMBER 1994

DND LANDFILL SOLID DISPOSED MASTE SUMMARY

RPT 6-1

DAD

(ALL VOLUMES $=$ CUBIC METERS)

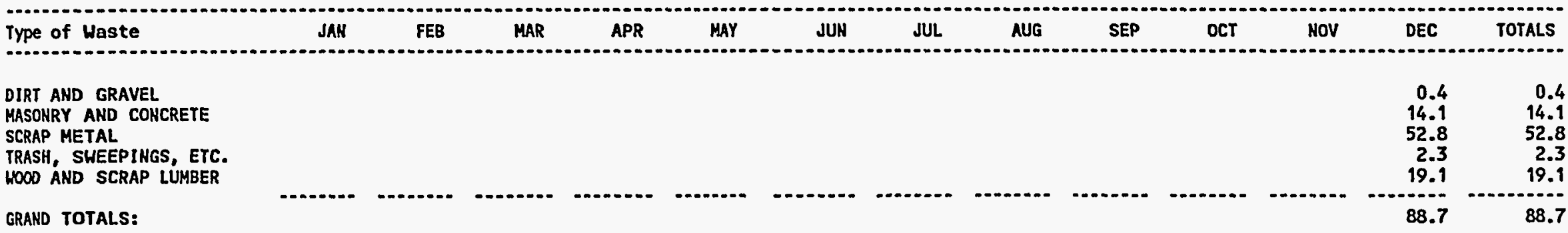

$\square$
$\emptyset$
$\dot{\omega}$

DETAILS MAY NOT ADD UP TO TOTALS BECAUSE OF ROUMDING

0.0 IH A COLUMH INDICATES A TOTAL OF $<0.05$ 
Naval Reactors Facility 1994 Detail Data

Report 184-NRF Airborne Release Point And Disposed Substances Summary .. NRF-3

Report 185-NRF Liquid Disposed Substances Summary .............. NRF-4

Report 185-NRF Release Point And Liquid Disposed Substances Summary ... . NRF-5

Report 186-NRF Landfill Solid Disposed Waste Summary . . . . . . . . NRF-7

Report 187-NRF Water Usage And Disposal Summary ............. NRF-8

Report 188-NRF Fuel Oil Usage And Stack Effluents Summary ......... NRF-10 
PAGE: 1

SCHED. NO. INRPTOOT
IDAHO OPERATIONS OFFICE

UNITED STATES DEPARTMENT OF ENERGY

INEL NONRADIOLOGICAL WASTE MANAGEMENT IHFORMATION SYSTEM

FOR JANUARY THROUGH DECEMBER 1994

NRF AIRBORNE RELEASE POINT AND DISPOSED SUBSTANCES SUMMARY

RPT 184-I

NRF

ANNUAL TOT
VOL/WE IGHT

RELEASE POINT DESCRIPTION

SUBSTANCE

JAN

FEB

MAR

APR

MAY

JUN

JUL

AUG

SEP

OCT

NOV

DEC

COOLING TOWERS

VOLUME (1000 CM)

20

3

6

7

7

7

4

8

3

3

5

78 
PAGE: 1

SCHED. NO. INRPTOOBA

IDAHO OPERATIONS OFFICE

26-JUL-95

DLOGICAL HASTE MANAGEMENT INFORMATION SYSTEM

FOR JANUARY THROUGH DECEMBER 1994

NRF LIQUID DISPOSED SUBSTANCES SUMMARY

RPT $185-$ I

NRF

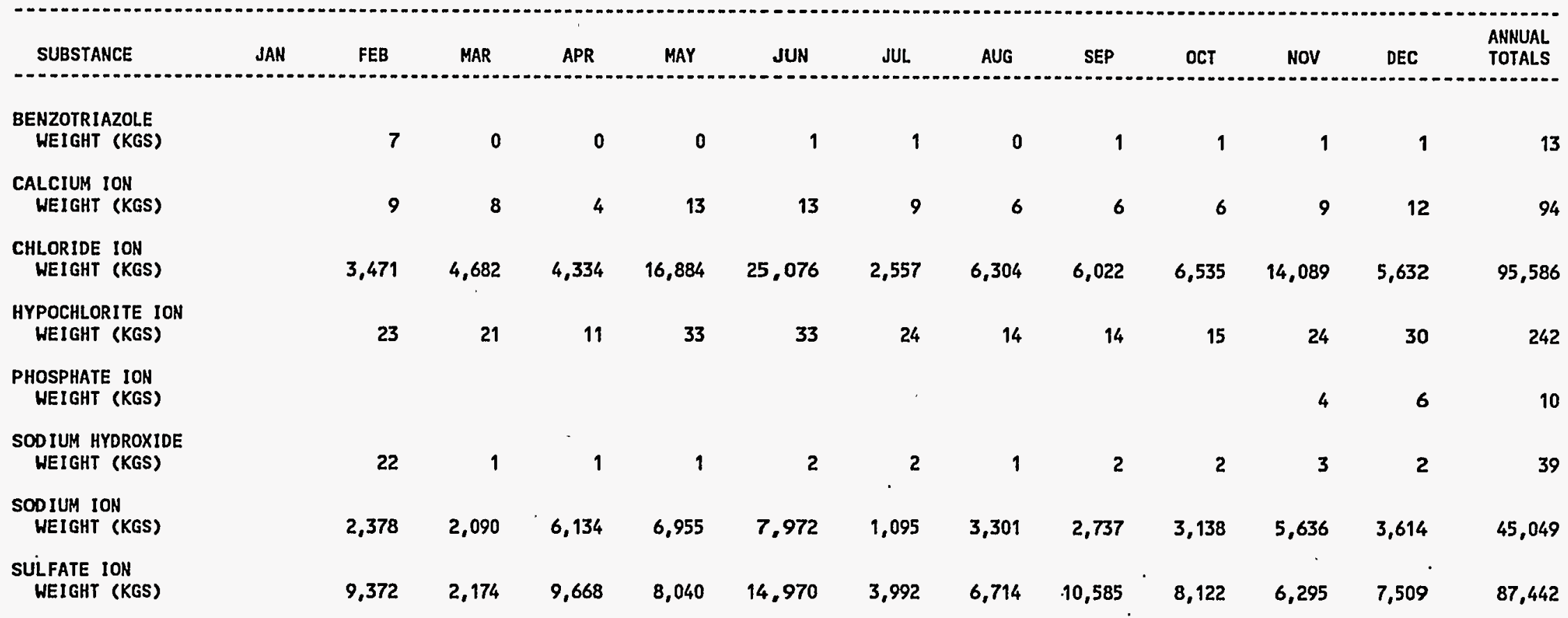




\begin{tabular}{|c|c|c|c|c|c|c|c|c|c|c|c|c|}
\hline $\begin{array}{l}\text { RELEASE POINT DESCRIPTION } \\
\text { SUBSTANCE JAN }\end{array}$ & FEB & MAR & APR & MAY & JUN & JUL & AUG & SEP & OCT & NOV & DEC & $\begin{array}{l}\text { ANNUAL TOT } \\
\text { VOL/WEIGHT } \\
\text { AVG CONC }\end{array}$ \\
\hline $\begin{array}{l}\text { SEWAGE POND } \\
\text { VOLUME (1000 L) } \\
\text { PH }\end{array}$ & $\begin{array}{r}3,324 \\
8.6\end{array}$ & $\begin{array}{r}3,642 \\
7.7\end{array}$ & $\begin{array}{r}4.047 \\
9.9\end{array}$ & $\begin{array}{r}4,558 \\
10.3\end{array}$ & $\begin{array}{r}4,474 \\
9.8\end{array}$ & $\begin{array}{r}4,849 \\
10.0\end{array}$ & $\begin{array}{r}4,872 \\
9.8\end{array}$ & $\begin{array}{r}3,780 \\
11.1\end{array}$ & $\begin{array}{r}2,951 \\
10.7\end{array}$ & $\begin{array}{r}5,050 \\
10.5\end{array}$ & $\begin{array}{r}5.224 \\
9.9\end{array}$ & $\begin{array}{r}50,471 \\
9.9\end{array}$ \\
\hline $\begin{array}{l}\text { HASTE DITCH } \\
\text { VOLUME }(1000 \mathrm{~L}) \\
\text { PH }\end{array}$ & $\begin{array}{r}34,712 \\
7.7\end{array}$ & $\begin{array}{r}33,444 \\
7.6\end{array}$ & $\begin{array}{r}33,338 \\
7.4\end{array}$ & $\begin{array}{r}40,201 \\
7.3\end{array}$ & $\begin{array}{r}37,426 \\
7.7\end{array}$ & $\begin{array}{r}44.857 \\
7.8\end{array}$ & $\begin{array}{r}51,254 \\
7.8\end{array}$ & $\begin{array}{r}36,499 \\
7.8\end{array}$ & $\begin{array}{r}36,919 \\
7.8\end{array}$ & $\begin{array}{r}29.977 \\
7.9\end{array}$ & $\begin{array}{r}23,466 \\
7.9\end{array}$ & $\begin{array}{r}463,606 \\
7.7\end{array}$ \\
\hline $\begin{array}{l}\text { BENZOTRIAZOLE } \\
\text { CONC. (MG/L) } \\
\text { HEIGHT (KGS) }\end{array}$ & $\begin{array}{r}0.21 \\
7\end{array}$ & $\begin{array}{r}0.01 \\
0\end{array}$ & $\begin{array}{r}0.09 \\
0\end{array}$ & $\begin{array}{r}0.01 \\
0\end{array}$ & $\begin{array}{r}0.01 \\
1\end{array}$ & $\begin{array}{r}0.02 \\
1\end{array}$ & $\begin{array}{r}0.01 \\
0\end{array}$ & $\begin{array}{r}0.02 \\
1\end{array}$ & $\begin{array}{r}0.02 \\
1\end{array}$ & $\begin{array}{r}0.03 \\
1\end{array}$ & $\begin{array}{r}0.03 \\
1\end{array}$ & $\begin{array}{r}0.03 \\
13\end{array}$ \\
\hline $\begin{array}{l}\text { CALCIUM ION } \\
\text { CONC. (MG/L) } \\
\text { HEIGHT (KGS) }\end{array}$ & $\begin{array}{r}0.25 \\
9\end{array}$ & $\begin{array}{r}0.25 \\
8\end{array}$ & $\begin{array}{r}0.12 \\
4\end{array}$ & $\begin{array}{r}0.32 \\
13\end{array}$ & $\begin{array}{r}0.35 \\
13\end{array}$ & $\begin{array}{r}0.21 \\
9\end{array}$ & $\begin{array}{r}0.11 \\
6\end{array}$ & $\begin{array}{r}0.15 \\
6\end{array}$ & $\begin{array}{r}0.16 \\
6\end{array}$ & $\begin{array}{r}0.31 \\
9\end{array}$ & $\begin{array}{r}0.49 \\
12\end{array}$ & $\begin{array}{r}0.20 \\
94\end{array}$ \\
\hline $\begin{array}{l}\text { CHLORIDE ION } \\
\text { CONC. (MG/L) } \\
\text { HEIGHT (KGS) }\end{array}$ & $\begin{array}{r}100.00 \\
3,471\end{array}$ & $\begin{array}{r}140.00 \\
4,682\end{array}$ & $\begin{array}{r}130.00 \\
4,334\end{array}$ & $\begin{array}{l}420.00 \\
16,884\end{array}$ & $\begin{array}{l}670.00 \\
25,076\end{array}$ & $\begin{array}{l}57.00 \\
2,557\end{array}$ & $\begin{array}{r}123.00 \\
6,304\end{array}$ & $\begin{array}{r}165.00 \\
6,022\end{array}$ & $\begin{array}{r}177.00 \\
6,535\end{array}$ & $\begin{array}{l}470.00 \\
14,089\end{array}$ & $\begin{array}{r}240,00 \\
5,632\end{array}$ & $\begin{array}{l}206.18 \\
95,586\end{array}$ \\
\hline $\begin{array}{l}\text { HYPOCHLORITE ION } \\
\text { CONC. (MG/L) } \\
\text { WEIGHT (KGS) }\end{array}$ & $\begin{array}{r}0.65 \\
23\end{array}$ & $\begin{array}{r}0.64 \\
21\end{array}$ & $\begin{array}{r}0.32 \\
11\end{array}$ & $\begin{array}{r}0.83 \\
33\end{array}$ & $\begin{array}{r}0.89 \\
33\end{array}$ & $\begin{array}{r}0.53 \\
24\end{array}$ & $\begin{array}{r}0.28 \\
14\end{array}$ & $\begin{array}{r}0.39 \\
14\end{array}$ & $\begin{array}{r}0.42 \\
15\end{array}$ & $\begin{array}{r}0.79 \\
24\end{array}$ & $\begin{array}{r}1.26 \\
30\end{array}$ & $\begin{array}{r}0.52 \\
242\end{array}$ \\
\hline $\begin{array}{l}\text { PHOSPHATE ION } \\
\text { CONC. (MG/L) } \\
\text { HEIGHT (KGS) }\end{array}$ & & & & & & & & & & $\begin{array}{r}0.15 \\
4\end{array}$ & $\begin{array}{r}0.25 \\
6\end{array}$ & $\begin{array}{r}0.02 \\
10\end{array}$ \\
\hline $\begin{array}{c}\text { SODIUM HYDROXIDE } \\
\text { CONC. (MG/L) } \\
\text { WEIGHT (KGS) }\end{array}$ & $\begin{array}{r}0.63 \\
22\end{array}$ & $\begin{array}{r}0.02 \\
1\end{array}$ & $\begin{array}{r}0.03 \\
1\end{array}$ & $\begin{array}{r}0.03 \\
1\end{array}$ & $\begin{array}{r}0.04 \\
2\end{array}$ & $\begin{array}{r}0.05 \\
2\end{array}$ & $\begin{array}{r}0.02 \\
1\end{array}$ & $\begin{array}{r}0.06 \\
2\end{array}$ & $\begin{array}{r}0.06 \\
2\end{array}$ & $\begin{array}{r}0.09 \\
3\end{array}$ & $\begin{array}{r}0.10 \\
2\end{array}$ & $\begin{array}{r}0.08 \\
39\end{array}$ \\
\hline
\end{tabular}


NRF LANDFILL SOLID DISPOSED WASTE SUMMARY

(ALL VOLUMES $=$ CUBIC METERS)

\begin{tabular}{|c|c|c|c|c|c|c|c|c|c|c|c|c|c|}
\hline Type of Waste & JAN & FEB & MAR & APR & MAY & JUN & JUL & AUG & SEP & OCT & NOV & DEC & TOTALS \\
\hline $\begin{array}{l}\text { ASBESTOS } \\
\text { ASPHALT } \\
\text { CAFETERIA GARBAGE } \\
\text { DIRT AND GRAVEL } \\
\text { MASONRY AND CONCRETE } \\
\text { SCRAP METAL } \\
\text { TRASH, SWEEPINGS, ETC. } \\
\text { WEEDS, GRASS AND TREES } \\
\text { WOOD AND SCRAP LUMBER }\end{array}$ & $\begin{array}{r}15.3 \\
671.3 \\
32.1\end{array}$ & $\begin{array}{r}21.4 \\
570.4 \\
5.4\end{array}$ & $\begin{array}{r}6.1 \\
78.8 \\
9.2 \\
3.1 \\
11.5 \\
708.0 \\
33.6\end{array}$ & $\begin{array}{r}108.6 \\
81.0 \\
34.4 \\
17.6 \\
22.2 \\
635.4 \\
27.5\end{array}$ & $\begin{array}{r}24.5 \\
53.5 \\
87.2 \\
7.6 \\
11.1 \\
22.2 \\
618.9 \\
6.1\end{array}$ & $\begin{array}{r}24.5 \\
20.6 \\
122.3 \\
26.0 \\
15.3 \\
532.2 \\
7.6 \\
8.4\end{array}$ & $\begin{array}{r}12.2 \\
22.2 \\
78.0 \\
7.6 \\
3.1 \\
6.1 \\
587.2 \\
12.2\end{array}$ & $\begin{array}{r}71.9 \\
8.4 \\
107.0 \\
9.9 \\
26.8 \\
12.6 \\
952.7 \\
\\
23.3\end{array}$ & $\begin{array}{r}45.9 \\
277.5 \\
93.3 \\
7.3 \\
35.9 \\
21.4 \\
645.3 \\
8.4\end{array}$ & $\begin{array}{r}0.8 \\
331.1 \\
72.6 \\
45.1 \\
15.7 \\
539.8 \\
9.9\end{array}$ & $\begin{array}{r}12.2 \\
269.9 \\
73.4 \\
22.9 \\
42.8 \\
17.2 \\
406.0 \\
13.0\end{array}$ & $\begin{array}{r}58.1 \\
1.5 \\
45.9 \\
0.8 \\
3.1 \\
3.8 \\
352.5 \\
6.1\end{array}$ & $\begin{array}{r}305.1 \\
1.099 .5 \\
1.002 .4 \\
99.8 \\
214.5 \\
184.7 \\
7,219.7 \\
7.6 \\
186.2\end{array}$ \\
\hline GRAND TOTALS: & 838.8 & 695.0 & 850.2 & 926.7 & 831.1 & 757.0 & 728.7 & 1.212 .7 & $1,135.0$ & $1,015.0$ & 857.5 & 471.8 & $10,319.4$ \\
\hline
\end{tabular}


(ALL VOLUMES $=$ MEAREST THOUSAND LITERS)

\begin{tabular}{|c|c|c|c|c|c|c|c|c|c|c|c|c|c|}
\hline $\begin{array}{l}\text { WELL OR BLDG. NO.I } \\
\text { SEWAGE BLDG. NO. }\end{array}$ & JAN & FEB & MAR & APR & MAY & JUN & JUL. & AUG & SEP & OCT & NOV & DEC & $\begin{array}{l}\text { ANNUAL TOT } \\
\text { VOL/WEIGHT } \\
\text { AVG CONC }\end{array}$ \\
\hline \multicolumn{14}{|l|}{ WATER DATA } \\
\hline $\begin{array}{l}001 \text { WATER PUMPED (L) } \\
002 \text { WATER PUMPED (L) } \\
003 \text { WATER PUMPED (L) } \\
004 \text { WATER PUMPED (L) }\end{array}$ & $\begin{array}{r}6,677 \\
51,784 \\
1,291 \\
25,184\end{array}$ & $\begin{array}{r}6,908 \\
12,465 \\
15 \\
21,827\end{array}$ & $\begin{array}{r}3,176 \\
10,039 \\
106 \\
29,159\end{array}$ & $\begin{array}{r}4,864 \\
10,308 \\
15 \\
28,250\end{array}$ & $\begin{array}{r}6,280 \\
9,672 \\
19 \\
35,511\end{array}$ & $\begin{array}{r}10,792 \\
9,751 \\
64 \\
28,023\end{array}$ & $\begin{array}{r}9,441 \\
10,800 \\
79 \\
36,586\end{array}$ & $\begin{array}{r}18,196 \\
8,237 \\
79 \\
34,072\end{array}$ & $\begin{array}{r}6,840 \\
8,506 \\
53 \\
32,789\end{array}$ & $\begin{array}{r}738 \\
9,066 \\
45 \\
33,130\end{array}$ & $\begin{array}{r}901 \\
9,229 \\
61 \\
27,739\end{array}$ & $\begin{array}{r}1,109 \\
10,804 \\
45 \\
21,721\end{array}$ & $\begin{array}{r}75,924 \\
160,660 \\
1,874 \\
353,992\end{array}$ \\
\hline HATER PUMPED (L) & 84,937 & 41,215 & 42,480 & 43,437 & 51,481 & 48,631 & 56,906 & 60,585 & 48,188 & 42,979 & 37,930 & 33,679 & 592,449 \\
\hline $\begin{array}{l}\text { WATER DISPOSED } \\
\text { AIR (L) } \\
\text { SURFACE (L) } \\
\text { SUBSURFACE (L) }\end{array}$ & $\begin{array}{l}19,714 \\
61,513\end{array}$ & $\begin{array}{r}3,182 \\
34,712\end{array}$ & $\begin{array}{r}5,390 \\
33,444\end{array}$ & $\begin{array}{r}6,049 \\
33,338\end{array}$ & $\begin{array}{r}6,712 \\
40,201\end{array}$ & $\begin{array}{r}6,730 \\
37,426\end{array}$ & $\begin{array}{r}7,207 \\
44,857\end{array}$ & $\begin{array}{r}4,455 \\
51,254\end{array}$ & $\begin{array}{r}7,911 \\
36,499\end{array}$ & $\begin{array}{r}3,918 \\
36,113\end{array}$ & $\begin{array}{r}2,903 \\
29,977\end{array}$ & $\begin{array}{r}4,989 \\
23,466\end{array}$ & $\begin{array}{r}79,161 \\
462,799\end{array}$ \\
\hline \multicolumn{14}{|l|}{ SEWAGE DATA } \\
\hline 722 BUILD VOL (L) & 3,701 & 3,324 & 3,642 & 4,047 & 4,558 & 4,474 & 4,849 & 4,872 & 3,780 & 2,951 & 5,050 & 5,224 & 50,471 \\
\hline $\begin{array}{l}\text { BIOCHEMICAL OXYGEN D } \\
\text { RAW CONC. (MG/L) } \\
\text { FINAL CONC. (MG/L) } \\
\% \text { REMOVED }\end{array}$ & $\begin{array}{c}\text { EMAND CBOD } \\
45.0\end{array}$ & 52.0 & 29.0 & 21.0 & 64.0 & 15.0 & 125.0 & 21.0 & 40.0 & 32.0 & 13.0 & 33.0 & 40.8 \\
\hline $\begin{array}{l}\text { DISSOLVED OXYGEN (DC } \\
\text { RAH CONC. (MG/L) } \\
\text { FINAL CONC. (MG/L) } \\
\text { INCREASED FACTOR }\end{array}$ & 0.8 & 1.6 & 2.2 & 13.9 & 14.9 & 11.4 & 5.1 & 8.7 & 17.6 & 12.4 & 11.5 & 0.8 & 8.4 \\
\hline \multicolumn{14}{|c|}{$\begin{array}{l}\text { SETTLEABLE SOLIDS (SS) } \\
\text { RAH CONC. (ML/L) } \\
\text { FINAL CONC. (ML/L) } \\
\text { \% REMOVED }\end{array}$} \\
\hline PH FINAL & 10.1 & 8.6 & 7.7 & 9.9 & 10.3 & 9.8 & 10.0 & 9.8 & 11.1 & 10.7 & 10.5 & 10.4 & 9.9 \\
\hline TOTAL SEWAGE(L) & 3,701 & 3,324 & 3,642 & 4,047 & 4,558 & 4,474 & 4,849 & 4,872 & 3,780 & 2,951 & 5,050 & 5,224 & 50,471 \\
\hline
\end{tabular}


PAGE: 2
SCHED. NO. INRPTO1O

IDAHO OPERATIONS OFFICE

UNITED STATES DEPARTMENT OF ENERGY

INEL NONRADIOLOGICAL WASTE MANAGEMENT INFORMATION SYSTEM

FOR JANUARY THROUGH DECEMBER 1994

NRF HATER USAGE AND DISPOSAL SUMMARY

RPT

$187-1$

(ALL VOLUMES = NEAREST THOUSAND LITERS)

ANNUAL TOT
VOL/WEIGHT

\begin{tabular}{|c|c|c|c|c|c|c|c|c|c|c|c|c|c|}
\hline $\begin{array}{l}\text { HELL OR BLDG. NO./ } \\
\text { SEWAGE BLDG. NO. }\end{array}$ & JAN & FEB & MAR & APR & MAY & JUN & JUL & AUG & SEP & OCT & NOV & DEC & $\begin{array}{l}\text { WNUAL TOT } \\
\text { OL/WEIGHT } \\
\text { AVG CONC }\end{array}$ \\
\hline TOTAL WATER DISPOSED(L) & 84,928 & 41,218 & 42,476 & 43,434 & 51,470 & 48,631 & 56,913 & 60,582 & 48,190 & 42,982 & 37,929 & 33,679 & 592,432 \\
\hline CCOUNTABILITY (\%) & 100.0 & 100.0 & 100.0 & 100.0 & 100.0 & 100.0 & 100.0 & 100.0 & 100.0 & 100.0 & 100.0 & 100.0 & 100.0 \\
\hline
\end{tabular}


PAGE: 1

SCHED. NO. INRPT011
IDAHO OPERATIONS OFFICE

27-JUL-95

UNITED STATES DEPARTMENT OF ENERGY

INEL. NONRADIOLOGICAL WASTE MANAGEMENT INFORMATION SYSTEM

FOR JANUARY THROUGH DECENBER 1994

NRF FUEL OIL USAGE AND STACK EFFLUENTS SUMMARY

RPT 188-I

NRF

(ALL VALUES = NEAREST THOUSAND)

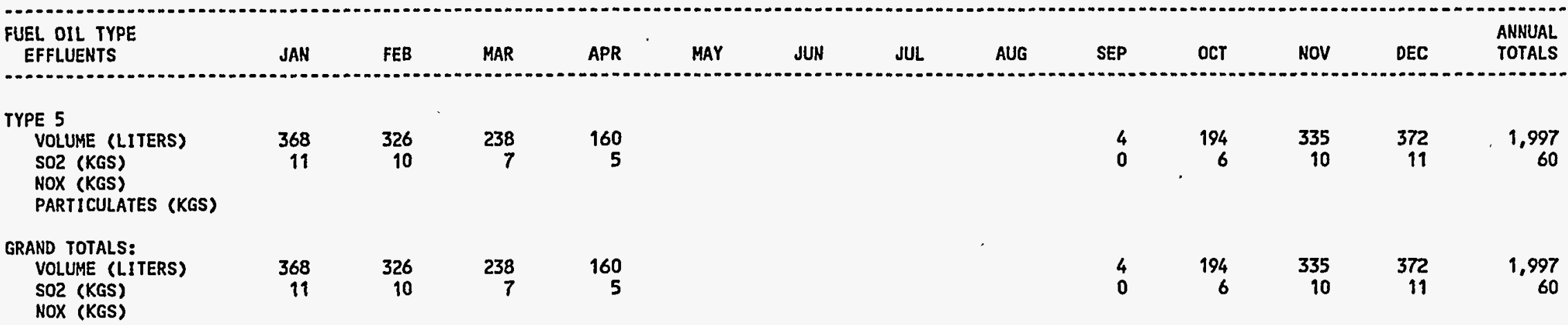

$O$ IN A COLUMN INDICATES A TOTAL OF $<0.5$ 
Power Burst Facility 1994 Detail Data

Report 195-PBF Liquid Disposed Substances Summary . . . . . . . . . . . PBF-3 Report 195-PBF Release Point And Liquid Disposed Substances Summary . . . PBF-6 Report 196_-PBF Landfill Solid Disposed Waste Summary . . . . . . . . . . . . PBF-11 Report 197-PBF Water Usage And Disposal Summary . . . . . . . . . . . . . . PBF-12 Report 198-PBF Fuel Oil Usage And Stack Effluents Summary . . . . . . . . . . PBF-14 
.

、 
PAGE: 1

SCHED. NO. INRPTOOBA

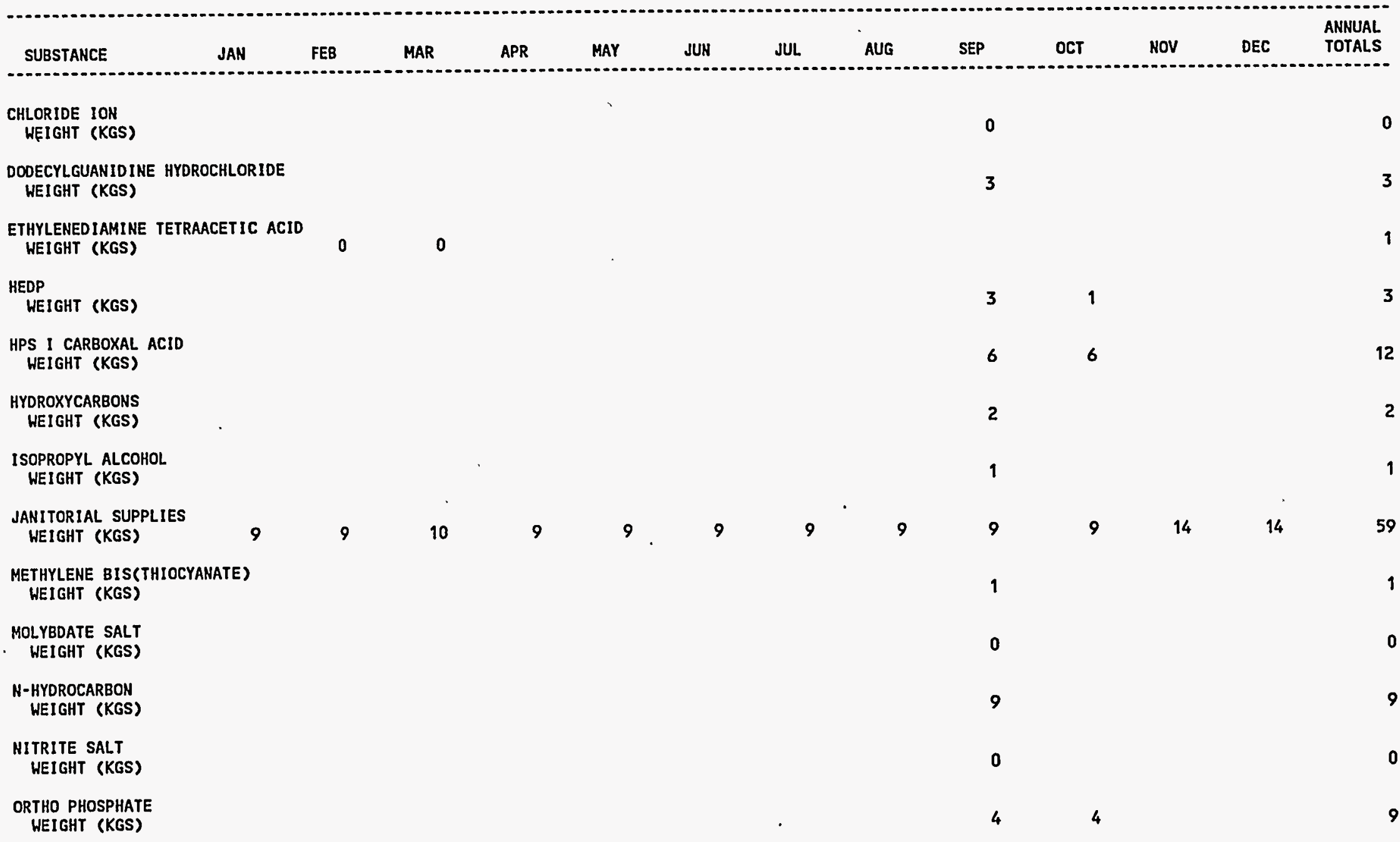

DETAILS MAY NOT ADD UP TO TOTALS BECAUSE OF ROUNDING

0 IN A COLUMM INDICATES A TOTAL OF $<0.5$ 
PAGE: 2

SCHED. NO. INRPTOOBA
IDAHO OPERATIONS OFFICE

27-JUL-95

UNITED STATES DEPARTMENT OF ENERGY

ULOGICAL WASTE MANAGEMENT INFORMATION SYSTEM

FOR JANUARY THROUGH DECEMBER 1994

PBF LIQUID DISPOSED SUBSTANCES SUMMARY

RPT 195-I

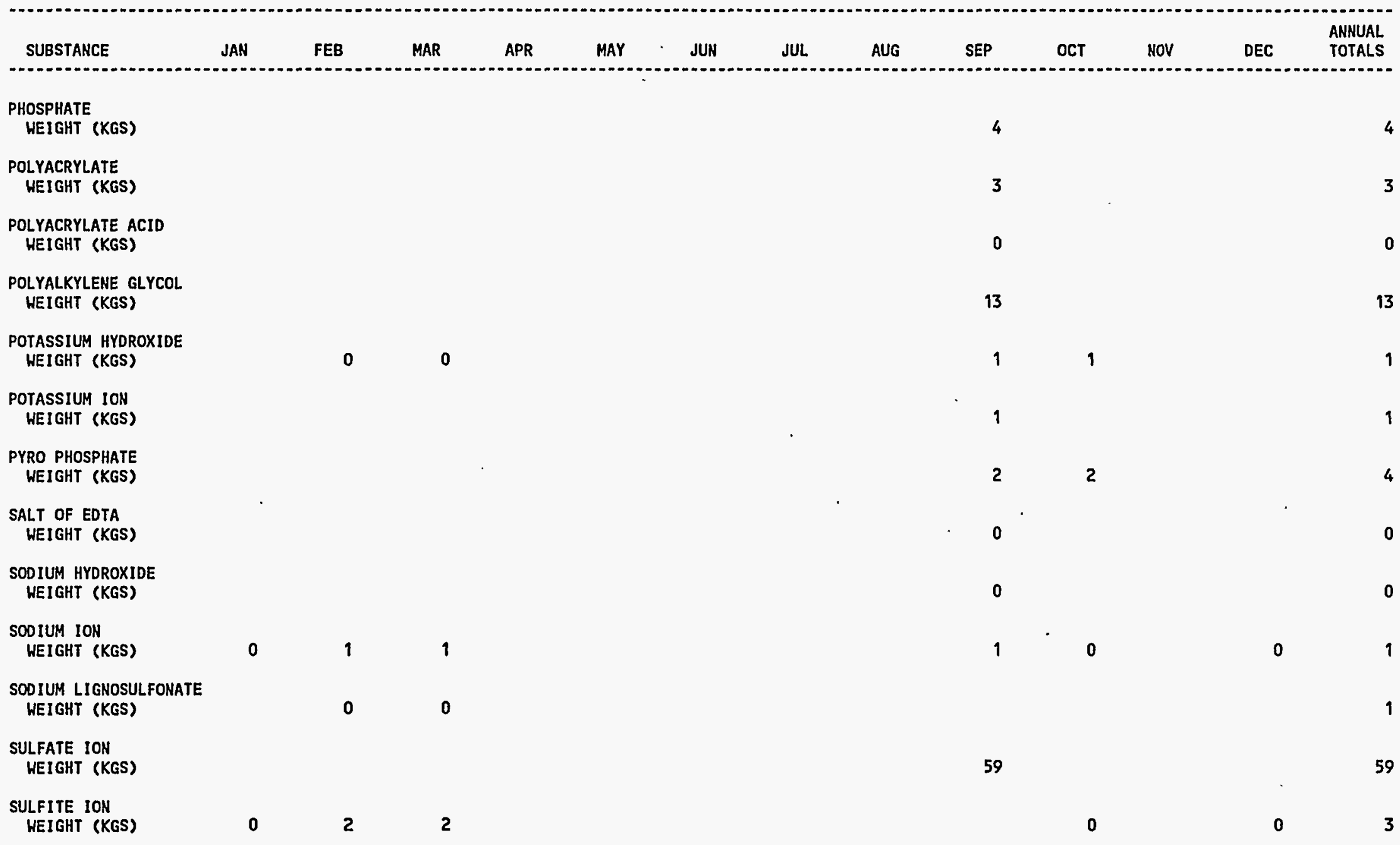

DETAILS MAY NOT ADD UP TO TOTALS BECAUSE OF ROUNDING

0 IN A COLUMN INDICATES A TOTAL OF $<0.5$ 
PAGE: 3

SCHED. NO. INRPTO08A

ION SYSTEM

FOR JANUARY THROUGH DECEMBER 1994

PBF LIQUIO DISPOSED SUBSTANCES SUMMARY

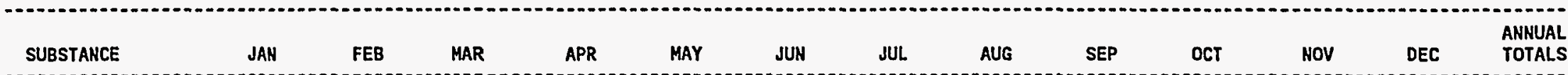

\section{TOLYTRIAZOLE}


INEL NONRADIOL OGICAL HASTE MANAGEMENT INFORMATION SYSTEM

FOR JANUARY THROUGH DECEMBER 1994

PBF RELEASE POINT AND LIQUID DISPOSED SUBSTANCES SUMMARY

RPT 195-I

PBF

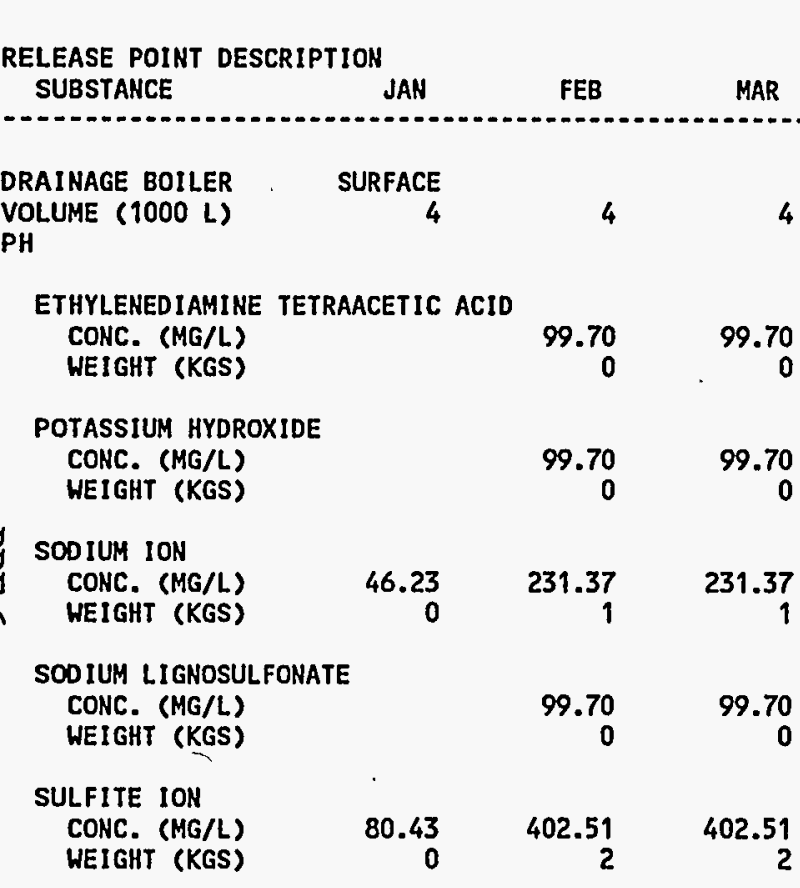

ANNUAL TOT

AVG CONC

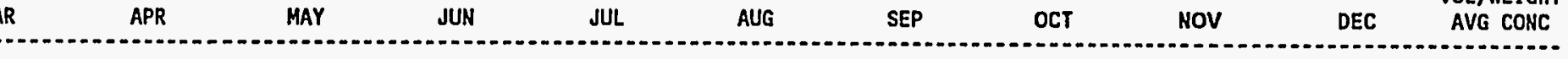

CHLORIDE ION

CONC. (MG/L) WEIGHT (KGS) 


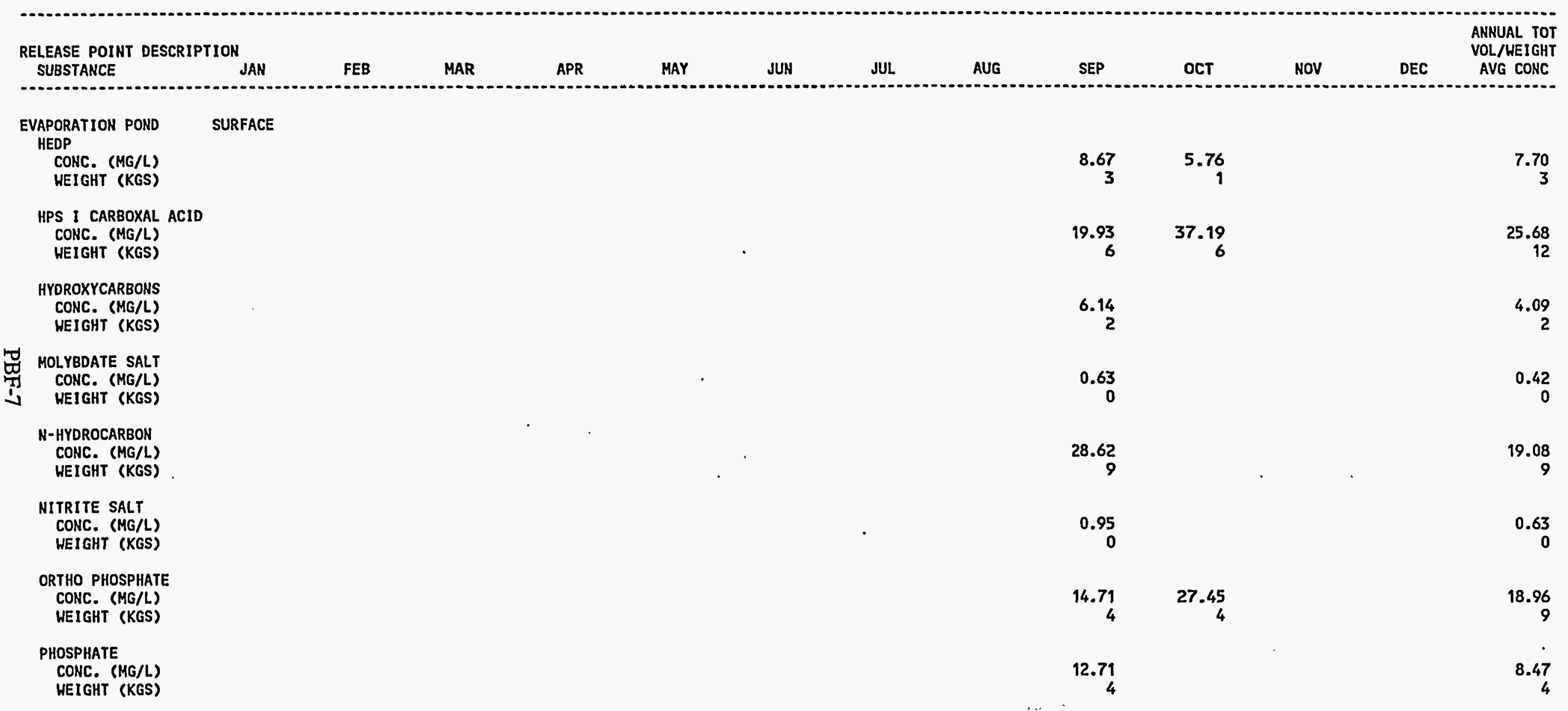




RELEASE POINT DESCRIPTION,
SUBSTANCE
EVAPORATION POND SURFACE
POLYCRYLATE
CONC. (MG/L)
WEIGHT (KGS)
POLYCRYLATE ACID
CONC. (MG/L)
WEIGHT (KGS)
POTASSIUM HYDROXIDE
CNC. CMG/L)
WEIGHT (KGS)
POTASSIUM ION
CONC. (MG/L)
WEIGHT (KGS)
PYRO PHOSPHATE
CONC. (MG/L)
WEIGHT (KGS)
SALT OF EDTA
CONC. (MG/L)
HEIGHT (KGS)
SODIUM HYROXIDE
CONC. (MG/L)
WEIGHT (KGS)
SODIUM ION
CONC. (MG/L)
HEIGHT (KGS)

11.51
3

0.06
0

0

2.37

4.43

2.01
1

7.35

13.73

0.06
0

0.06
0

3.14

ANNUAL TOT

VOL/WEIGHT

AVG CONC

DETAILS MAY NOT ADD UP TO TOTALS BECAUSE OF ROUNDING

0 IN A COLUMN INDICATES A TOTAL OF $<0.5$

0.00 IN A COLUMN INDICATES A TOTAL OF $<0.005$ 


\begin{tabular}{|c|c|c|c|c|c|c|c|c|c|c|c|c|}
\hline $\begin{array}{l}\text { RELEASE POINT DESCRIPTION } \\
\text { SUBSTANCE JAN }\end{array}$ & FEB & MAR & APR & MAY & JUN & JUL & AUG & SEP & ОСТ & NOV & DEC & $\begin{array}{l}\text { VOL/WEIGHT } \\
\text { AVG CONC }\end{array}$ \\
\hline $\begin{array}{l}\text { EVAPORATION POND } \\
\text { SUL.FATE ION } \\
\text { CONC. (MG/L) } \\
\text { WEIGHT (KGS) }\end{array}$ & & & & & & & & $\begin{array}{r}194.29 \\
59\end{array}$ & & & & $\begin{array}{r}129.53 \\
59\end{array}$ \\
\hline $\begin{array}{l}\text { TOLYTRIAZOLE } \\
\text { CONC. (MG/L) } \\
\text { HEIGHT (KGS) }\end{array}$ & & & & & & & & $\begin{array}{r}5.26 \\
2\end{array}$ & $\begin{array}{r}7.08 \\
1\end{array}$ & & & 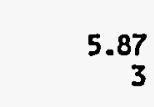 \\
\hline $\begin{array}{lc}\text { SEPTIC TANK (PBF) } & \text { SUBSURFACE } \\
\text { VOLUME }(1000 \mathrm{~L}) & 239 \\
\text { PH } & \end{array}$ & 376 & 428 & 704 & 615 & 1,465 & 401 & 876 & 575 & 505 & 321 & 458 & 6,9 \\
\hline $\begin{array}{l}\text { DODECYLGUANIDINE HYDROCHLORIDE } \\
\text { CONC. (MG/L) } \\
\text { WEIGHT (KGS) }\end{array}$ & & & & & & & & $\begin{array}{r}5.18 \\
3\end{array}$ & & & & 0 \\
\hline $\begin{array}{l}\text { ISOPROPYL ALCOHOL } \\
\text { CONC. (MG/L) } \\
\text { WEIGHT (KGS) }\end{array}$ & & & & & & & & $\begin{array}{r}1.73 \\
1\end{array}$ & & . & & 0 \\
\hline $\begin{array}{lr}\text { JANITORIAL SUPPLIES } \\
\text { CONC. (MG/L) } & 18.99 \\
\text { HEIGHT (KGS) } & 5\end{array}$ & $\begin{array}{r}12.05 \\
5\end{array}$ & $\begin{array}{r}13.77 \\
6\end{array}$ & $\begin{array}{r}6.45 \\
5\end{array}$ & $\begin{array}{r}7.37 \\
5\end{array}$ & $\begin{array}{r}3.10 \\
5\end{array}$ & $\begin{array}{r}11.32 \\
5\end{array}$ & $\begin{array}{r}5.18 \\
5\end{array}$ & $\begin{array}{r}7.88 \\
5\end{array}$ & $\begin{array}{r}8.99 \\
5\end{array}$ & $\begin{array}{r}21.19 \\
7\end{array}$ & $\begin{array}{r}14.87 \\
7\end{array}$ & \\
\hline $\begin{array}{l}\text { METHYLENE BIS(THIOCYANATE) } \\
\text { CONC. (MG/L) } \\
\text { WEIGHT (KGS) }\end{array}$ & & & & & & & & $\begin{array}{r}1.73 \\
1\end{array}$ & * & & & 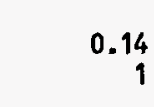 \\
\hline $\begin{array}{l}\text { POLYALKYLENE GLYCOL } \\
\text { CONC. (MG/L) } \\
\text { WEIGHT (KGS) }\end{array}$ & & & & & & - & $\ldots$ & $\begin{array}{r}22.44 \\
\ldots \quad 13\end{array}$ & & & & $\begin{array}{l}5 \\
3\end{array}$ \\
\hline
\end{tabular}

SEPTIC TANK (WERF) SUBSURFACE 
PAGE: 5

SCHED. NO. INRPTOOS
IDAHO OPERATIONS OFFICE

27-JUL-95

UNITED STATES DEPARTMENT OF ENERGY

INEL NONRADIOLOGICAL WASTE MANAGEMENT INFORMATION SYSTEM

FOR JANUARY THROUGH DECEMBER 1994

PBF RELEASE POINT AND LIQUID DISPOSED SUBSTANCES SUMMARY

RPT 195-I

PBF

\begin{tabular}{|c|c|c|c|c|c|c|c|c|c|c|c|c|c|}
\hline $\begin{array}{l}\text { ELEASE POINT DESCRIP } \\
\text { SUBSTANCE }\end{array}$ & 'TION JAN & FEB & MAR & APR & MAY & JUN & JUL & AUG & SEP & OCT & NOV & DEC & $\begin{array}{l}\text { ANNUAL TOT } \\
\text { VOL/WEIGHT } \\
\text { AVG CONC }\end{array}$ \\
\hline $\begin{array}{l}\text { EPTIC TANK (WERF) } \\
\text { OLUME }(1000 \mathrm{~L})\end{array}$ & $\begin{array}{c}\text { SUBSURFACE } \\
119\end{array}$ & 188 & 214 & 352 & 307 & 732 & 201 & 438 & 288 & 252 & 161 & 229 & 3,481 \\
\hline $\begin{array}{l}\text { JANITORIAL SUPPLIES } \\
\text { CONC. (MG/L) } \\
\text { WEIGHT (KGS) }\end{array}$ & $\begin{array}{r}37.98 \\
5\end{array}$ & $\begin{array}{r}24.10 \\
5\end{array}$ & $\begin{array}{r}21.19 \\
5\end{array}$ & $\begin{array}{r}12.89 \\
5\end{array}$ & $\begin{array}{r}14.75 \\
5\end{array}$ & $\begin{array}{r}6.19 \\
5\end{array}$ & $\begin{array}{r}22.62 \\
5\end{array}$ & $\begin{array}{r}10.36 \\
5\end{array}$ & $\begin{array}{r}15.77 \\
5\end{array}$ & $\begin{array}{r}17.98 \\
5\end{array}$ & $\begin{array}{r}42.38 \\
7\end{array}$ & $\begin{array}{r}29.73 \\
7\end{array}$ & $\begin{array}{r}16.94 \\
59\end{array}$ \\
\hline
\end{tabular}

DETAILS MAY NOT ADD UP TO TOTALS BECAUSE OF ROUNDING

0 IN A COLUMN INDICATES A TOTAL OF $<0.5$

0.00 IN A COLUMN INDICATES A TOTAL OF $<0.005$ 
PAGE: 1

SCHED. NO. INRPTOO9
IDAHO OPERATIONS OFFICE

27-JUL-95

UNITED STATES DEPARTMENT OF ENERGY

INEL. HONRADIOLOGICAL WASTE MANAGEMENT IMFORMATION SYSTEM

FOR JANUARY THROUGH DECEMBER 1994

PBF LANDFILL SOLID DISPOSED HASTE SUMMARY

RPT 196-I

PBF

(ALL VOLUMES = CUBIC METERS)

\begin{tabular}{|c|c|c|c|c|c|c|c|c|c|c|c|c|c|}
\hline Type of Waste & JAN & FEB & MAR & APR & MAY & JUN & JUL & AUG & SEP & OCT & NOV & DEC & TOTALS \\
\hline $\begin{array}{l}\text { ASPHALT } \\
\text { DIRT AND GRAVEL } \\
\text { MASONRY AND CONCRETE } \\
\text { SCRAP METAL } \\
\text { TRASH, SWEEPINGS, ETC. } \\
\text { WEEDS, GRASS AND TREES } \\
\text { HOOD AND SCRAP LUMBER }\end{array}$ & $\begin{array}{r}1.9 \\
84.9 \\
5.0\end{array}$ & $\begin{array}{r}0.4 \\
47.4 \\
5.4\end{array}$ & $\begin{array}{r}0.4 \\
3.1 \\
81.0 \\
3.4\end{array}$ & $\begin{array}{r}0.4 \\
1.5 \\
63.5 \\
0.4 \\
2.3\end{array}$ & $\begin{array}{r}5.4 \\
2.7 \\
52.8 \\
3.1\end{array}$ & $\begin{array}{r}35.9 \\
0.8\end{array}$ & $\begin{array}{r}37.1 \\
1.1 \\
0.4\end{array}$ & $\begin{array}{r}8.8 \\
73.0 \\
0.8 \\
0.4\end{array}$ & $\begin{array}{r}0.4 \\
40.1 \\
0.4\end{array}$ & $\begin{array}{r}10.7 \\
0.4 \\
5.4 \\
53.1 \\
0.4 \\
3.1\end{array}$ & $\begin{array}{r}0.4 \\
41.3 \\
1.1\end{array}$ & $\begin{array}{r}2.3 \\
35.9 \\
0.4\end{array}$ & $\begin{array}{r}12.2 \\
10.7 \\
6.5 \\
26.8 \\
645.3 \\
2.7 \\
25.6\end{array}$ \\
\hline
\end{tabular}

DETAILS MAY NOT ADD UP TO TOTALS BECAUSE OF ROUNDING

0.0 IN A COLUMN INDICATES A TOTAL OF $<0.05$ 
FOR JANUARY THROUGH DECEMBER 1994

PBF WATER USAGE AND DISPOSAL SUMMARY

RPT 197-I

PBF

(ALL VOLUMES = NEAREST THOUSAND LITERS)

ANUUAL TOT VOL/WEIGHT

WELL OR BLDG. NO./
SEWAGE BLDG. NO.

WATER DATA

001 WATER PUMPED (L)
002 WATER PUMPED (L)

WATER PUMPED (L)

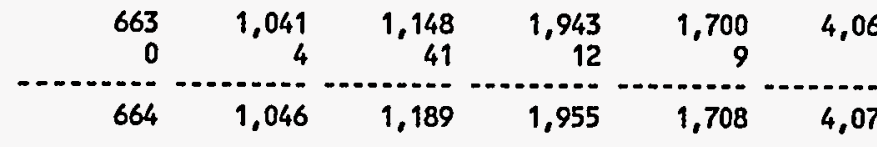

1,114
401
1,515

\begin{tabular}{rr}
203 & 50 \\
2,230 & 1,547 \\
\hline 2,433 & 1,598
\end{tabular}

\begin{tabular}{rrrr}
1,384 & 546 & 572 & 14,434 \\
18 & 346 & 700 & 5,308 \\
\hline 1,401 & 892 & 1,272 & 19,742
\end{tabular}

WATER DISPOSED

\section{AIR (L)}

SURFACE (L)

SUBSURFACE (L)

199

314

357

586

513

1,221

334

730

479

421

268

385

5,806

SEHAGE DATA

619 BUILD VOL (L)

188

214

BIOCHEMICAL OXYGEN DEMAND (BOD)

RAW CONC. (MG/L)

FINAL CONC. (MG/L)

$\%$. REMOVED

DISSOLVED OXYGEN (DO)

RAH CONC. (MG/L)

FINAL CONC. (MG/L)

INCREASED FACTOR

SETTLEABLE SOLIDS (SS)

RAH CONC. (ML/L)

FINAL CONC. (ML/L)

\% REMOVED

PH FINAL.

DETAILS MAY NOT ADD UP TO TOTALS BECAUSE OF ROUNDING

$O$ IN A COLUMN INDICATES A TOTAL OF $<0.5$

0.0 IN A COLUMN INDICATES A TOTAL OF $<0.05$

0.00 IN A COLUMM INDICATES A TOTAL OF $<0.005$ 
PAGE: 2

SCHED. NO. INRPT010
IDAHO OPERATIONS OFFICE

UNITED STATES DEPARTMENT OF ENERGY

27-JUL-95
FOR JANUARY THROUGH DECEMBER 1994
PBF WATER USAGE AND DISPOSAL. SUMMARY
RPT 197-I

PBF

(ALL VOLUMES = NEAREST THOUSAND LITERS)

HELL OR BLDG. NO.J
SEHAGE BLDG. NO. JAN FEB MAR APR MAY JUN JUL AUE SEP OCT NOV AVG CONC

SEWAGE BLDG. NO.

239

376

428

704

$615 \quad 1,465$

401

876

575

505

321

458

6,963

BIOCHEMICAL OXYGEN DEMAND (BOD)

RAW CONC. (MG/L)

FINAL CONC. (MG/L)

\% REMOVED

DISSOLVED OXYGEN (DO)

RAW CONC. (MG/L)

FINAL CONC. (MG/L)

INCREASED FACTOR

SETTLEABLE SOLIDS (SS)

RAW CONC. (ML/L)

FINAL CONC. (ML/L)

\% REMOVED

PH FINAL

TOTAL SEWAGE(L)

358

565

.642

1,056

923

2,198

601

1,314

863

757

482

$687 \quad 10,444$

TOTAL WATER DISPOSED(L)

557

1,642

$1,435 \quad 3,419$

$936 \quad 2,044$

1,342

1,177

749

$1,07216,251$

ACCOUNTABILITY (\%)

$84.0 \quad 84.0$

$84.0 \quad 84.0$

$84.0 \quad 84.0$

$61.8 \quad 84.0$

84.0

84.0

84.0

84.3

82.3

DETAILS MAY NOT ADD UP TO TOTALS BECAUSE OF ROUNDING

0 IN A COLUMN INDICATES A TOTAL OF $<0.5$

0.0 IN A COLUMN INDICATES A TOTAL OF $<0.05$

0.00 IN A COLUMN INDICATES A TOTAL OF $<0.005$ 
PAGE: 1

SCHED. NO. INRPTO11
IDAHO OPERATIONS OFFICE

UNITED STATES DEPARTMENT OF ENERGY

27-JUL-95

INEL. NONRADIOLOGICAL WASTE MANAGEMENT INFORMATION SYSTEM

FOR JANUARY THROUGH DECEMBER 1994

PBF FUEL OIL USAGE AND STACK EFFLUENTS SUMMARY

RPT 198-1

PBF

(ALL VALUES $=$ NEAREST THOUSAND)

FUEL OIL TYPE
EFFLUENTS

TYPE 2

VOLUME (LITERS)

SOR (KGS)

NOX (KGS)

PARTICULATES (KGS)

GRAND TOTALS:

VOLUME (LITERS)

SO2 (KGS)

20

20
0

19
0

APR

MAY

JUN

JUL

AUG

SEP

OCT

NOV

DEC

ANNUAL

TOTALS

PARTICULATES (KGS)

莗
$\frac{1}{+}$ 
Radioactive Waste Management Complex 1994 Detail Data

Report 256-WMC Landfill Solid Disposed Waste Summary ........... WMC-3

Report 257-WMC Water Usage And Disposal Summary . . . . . . . . . WMC-4 
PAGE: 1

SCHED. NO. INRPTOO9
IDAHO OPERATIONS OFFICE

UNITED STATES DEPARTMEHT OF ENERGY

INEL NONRADIOLOGICAL WASTE MANAGEMEMT IHFORMATION SYSTEM

FOR JANUARY THROUGH DECEMBER 9994

WMC LANDFILL SOLID DISPOSED MASTE SUMMARY
27-JUL-95

RPT 256-1

(ALL VOLUMES = CUBIC METERS)

\begin{tabular}{|c|c|c|c|c|c|c|c|c|c|c|c|c|c|}
\hline Type of Waste & JAN & FEB & MAR & APR & MAY & JUN & JUL & AUG & SEP & DCT & NOV & DEC & TOTALS. \\
\hline $\begin{array}{l}\text { DIRT AND GRAVEL } \\
\text { MASONRY AND CONCRETE } \\
\text { SCRAP METAL } \\
\text { TRASH, SWEEPINGS, ETC. } \\
\text { WEEDS, GRASS AND TREES } \\
\text { WOOD AND SCRAP LUMBER }\end{array}$ & 61.6 & 61.2 & $\begin{array}{r}4.2 \\
73.8 \\
8.0\end{array}$ & $\begin{array}{r}3.1 \\
68.8\end{array}$ & $\begin{array}{r}0.4 \\
57.0 \\
0.4\end{array}$ & $\begin{array}{r}3.1 \\
58.9 \\
16.8\end{array}$ & $\begin{array}{r}3.1 \\
79.9 \\
1.1\end{array}$ & $\begin{array}{r}0.8 \\
80.3 \\
8.0\end{array}$ & $\begin{array}{r}6.1 \\
92.9 \\
11.5\end{array}$ & $\begin{array}{r}48.6 \\
2.3 \\
9.2\end{array}$ & $\begin{array}{r}1.9 \\
55.4\end{array}$ & 71.9 & $\begin{array}{r}155.2 \\
1.9 \\
20.6 \\
810.1 \\
2.3 \\
55.1\end{array}$ \\
\hline GRAND TOTALS: & 61.6 & 61.2 & 86.0 & 71.9 & 57.7 & 78.8 & 84.1 & 89.1 & 110.5 & 215.2 & 57.3 & 71.9 & $1,045.2$ \\
\hline
\end{tabular}


PAGE: 1

SCHED. NO. INRPT010
IDAHO OPERATIONS OFFICE

UNITED STATES DEPARTMENT OF ENERGY

$27-$ JUL -95

INEL NONRADIOLOGICAL. HASTE MANAGEMENT IMFORMATION SYSTEM

FOR JANUARY THROUGH DECEMBER 1994

WMC HATER USAGE AND DISPOSAL SUMMARY

RPT 257-1

HMC

(ALL. VOLUMES = NEAREST THOUSAND LITERS)

ANNUAL TO

VOL/WEIGHT

WELL OR BLDG. NO./

JAN FEB

MAR

APR

MAY

JUN

JUL

AUG

SEP

OCT

NOV DEC AVG CONC

\section{WATER DATA}

WMC WATER PUMPED (L)

\begin{tabular}{|c|c|c|c|c|c|c|c|c|c|c|c|c|}
\hline 501 & 859 & 2,257 & 798 & 2,068 & 5,243 & 12,988 & 4,864 & 3,183 & 1,868 & 746 & 1,163 & 36,537 \\
\hline 501 & 859 & 2,257 & 798 & 2,068 & 5,243 & 12,988 & 4,864 & 3,183 & 1,868 & 746 & 1,163 & 36,537 \\
\hline
\end{tabular}

WATER PUMPED (L)

WATER DISPOSED

$$
\begin{aligned}
& \text { AIR (L) } \\
& \text { SURFACE (L) } \\
& \text { SUBSURFACE (L) }
\end{aligned}
$$

SEWAGE DATA

WMC BUILD VOL (L)

85

146

161

142

150

157

79

119

104

104

266

253

1,767

RAW CONC. (MG/L)

FINAL CONC. (MG/L)

\% REMOVED

DISSOLVED OXYGEN (DO)

RAW CONC. (MG/L)

FINAL CONC. (MG/L)

INCREASED FACTOR

SETTLEABLE SOLIDS (SS)

RAH CONC. (ML/L)

FINAL CONC. (ML/L)

\% REMOVED

PH FINAL

TOTAL SEWAGE(L)

$85 \quad 146$

146

$161 \quad 142$

150

$\begin{array}{ll}157 & 79 \\ 157 & 79\end{array}$

119

104

104
104

266

$\begin{array}{ll}253 & 1,767 \\ 253 & 1.767\end{array}$

DETAILS MAY NOT ADD UP TO TOTALS BECAUSE OF ROUNDING

0 IN A COLUMN INDICATES A TOTAL OF $<0.5$

0.0 IN A COLUMN INDICATES A TOTAL OF $<0.05$

0.00 IN A COLUMN INDICATES A TOTAL OF $<0.005$ 
PAGE: 2
SCHED. NO. INRPTO10

IDAHO OPERATIONS OFFICE

UNITED STATES DEPARTMENT OF ENERGY

27-JUL-95

INEL NONRADIOLOGICAL WASTE MANAGEMENT INFORMATION SYSTEM

FOR JANUARY THROUGH DECEMBER 1994

WMC WATER USAGE AND DISPOSAL SUMMARY

RPT 257-1

MMC

(ALL VOLUMES = NEAREST THOUSAND LITERS)

ANNUAL TOT

HELL OR BLDG. NO.

SEWAGE BLDG. NO.

JAN FEB MAR APR

PR $\quad M A Y$

MAY JUN JUL

SEP

ACCOUNTABILITY (\%)

17.0

17.0

7.2

17.8

7.2

3.0

0.6

2.4

3.3

21.8

4.8 
Special Manufacturing Capability 1994 Detail Data

Report 278-SMC Fuel Oil Usage And Stack Effluents Summary .......... SMC-3 
PAGE: 1
SCHED. NO. INRPTOI1

IDAHO OPERATIONS OFFICE

IOLOGICAL WASTE MANAGEMENT INFORMATION SYSTEM

FOR JANUARY THROUGH DECENBER 1994

SMC FUEL OIL USAGE AND STACK EFFLUENTS SUMMARY

(ALL VALUES = NEAREST THOUSAND)

\begin{tabular}{|c|c|c|c|c|c|c|c|c|c|c|c|c|c|}
\hline $\begin{array}{l}\text { FUEL OIL TYPE } \\
\text { EFFLUENTS }\end{array}$ & JAN & FEB & HAR & APR & MAY & JUN & JUL & AUG & SEP & OCT & NOV & DEC & $\begin{array}{l}\text { ANNUAL } \\
\text { TOTALS }\end{array}$ \\
\hline $\begin{array}{l}\text { TYPE } 2 \\
\text { VOLUME (LITERS) } \\
\text { SO2 (KGS) } \\
\text { NOX (KGS) } \\
\text { PARTICULATES (KGS) }\end{array}$ & $\begin{array}{r}228 \\
1\end{array}$ & $\begin{array}{r}253 \\
1\end{array}$ & $\begin{array}{r}216 \\
1\end{array}$ & $\begin{array}{r}125 \\
1\end{array}$ & $\begin{array}{r}96 \\
0\end{array}$ & $\begin{array}{r}91 \\
0\end{array}$ & $\begin{array}{r}62 \\
0\end{array}$ & $\begin{array}{r}29 \\
0\end{array}$ & $\begin{array}{r}82 \\
0\end{array}$ & $\begin{array}{r}121 \\
1\end{array}$ & $\begin{array}{r}197 \\
1\end{array}$ & $\begin{array}{r}228 \\
1\end{array}$ & $\begin{array}{r}1,728 \\
9\end{array}$ \\
\hline $\begin{array}{l}\text { GRAND TOTALS: } \\
\text { VOLUME (LITERS) } \\
\text { SO2 (KGS) } \\
\text { NOX (KGS) }\end{array}$ & $\begin{array}{r}228 \\
1\end{array}$ & $\begin{array}{r}253 \\
1\end{array}$ & $\begin{array}{r}216 \\
1\end{array}$ & $\begin{array}{r}125 \\
1\end{array}$ & $\begin{array}{r}96 \\
0\end{array}$ & $\begin{array}{r}91 \\
0\end{array}$ & $\begin{array}{r}62 \\
0\end{array}$ & $\begin{array}{r}29 \\
0\end{array}$ & $\begin{array}{r}82 \\
0\end{array}$ & 121 & $\begin{array}{r}197 \\
q\end{array}$ & $\begin{array}{r}228 \\
1\end{array}$ & $\begin{array}{r}1,728 \\
9\end{array}$ \\
\hline
\end{tabular}




\section{Test Area North 1994 Detail Data}

Report 216-TAN Landfill Solid Disposed Waste Summary . . . . . . . . . . . . TAN-3 
PAGE:

SCHED. NO. INRPTOO9
IDAHO OPERATIONS OFFICE

UNITED STATES DEPARTMENT OF ENERGY

INEL. NONRADIOLOGICAL HASTE MANAGEMENT IHFORMATION SYSTEH

FOR JANUARY THROUGH DECEMBER 1994

TAN LANDFILL SOLID DISPOSED HASTE SUMMARY
$27-J U L-95$

RPT 216-I

TAN

(ALL VOLUMES = CUBIC METERS)

\begin{tabular}{|c|c|c|c|c|c|c|c|c|c|c|c|c|c|}
\hline Type of Waste & JAN & FEB & MAR & APR & MAY & JUN & JUL & AUG & SEP & OCT & Nov & DEC & TOTALS \\
\hline $\begin{array}{l}\text { ASBESTOS } \\
\text { ASPHALT } \\
\text { CAFETERIA GARBAGE } \\
\text { DIRT AND GRAVEL } \\
\text { MASONRY AND CONCRETE } \\
\text { SCRAP METAL } \\
\text { TRASH, SWEEPINGS, ETC. } \\
\text { WOOD AND SCRAP LUMBER }\end{array}$ & $\begin{array}{r}18.4 \\
3.1\end{array}$ & $\begin{array}{r}24.5 \\
18.4 \\
182.0\end{array}$ & $\begin{array}{r}6.5 \\
177.0 \\
6.1\end{array}$ & $\begin{array}{r}21.8 \\
209.1\end{array}$ & $\begin{array}{r}5.4 \\
2.3 \\
2.3 \\
43.6 \\
219.4 \\
7.6\end{array}$ & $\begin{array}{r}9.2 \\
69.6 \\
200.3 \\
6.1\end{array}$ & $\begin{array}{r}0.8 \\
18.4 \\
152.9\end{array}$ & $\begin{array}{r}9.2 \\
42.8 \\
14.1 \\
203.0 \\
29.1\end{array}$ & $\begin{array}{r}36.7 \\
12.2 \\
\\
29.1 \\
315.8 \\
50.5 \\
206.4 \\
6.1\end{array}$ & $\begin{array}{r}13.8 \\
7.6 \\
214.9 \\
6.1\end{array}$ & $\begin{array}{r}12.2 \\
7.6 \\
217.9 \\
13.8\end{array}$ & $\begin{array}{r}3.4 \\
133.8 \\
0.4\end{array}$ & $\begin{array}{r}64.2 \\
48.2 \\
9.2 \\
34.4 \\
416.7 \\
253.8 \\
2.298 .0 \\
88.3\end{array}$ \\
\hline ND TOTALS: & 215.6 & & 189.6 & 230.9 & 280.6 & 285.2 & 172.0 & 298.2 & 656.8 & & 251.6 & 149.9 & 3,21 \\
\hline
\end{tabular}




\section{Test Reactor Area 1994 Detail Data}

Report 235-TRA Liquid Disposed Substances Summary . . . . . . . . . . . . TRA-3

Report 235-TRA Release Point And Liquid Disposed Substances Summary . . . TRA-6

Report 236 -TRA Landfill Solid Disposed Waste Summary . . . . . . . . . . . . TRA-12

Report 237-TRA Water Usage And Disposal Summary . . . . . . . . . . . . . . TRA-13

Report 238-TRA Fuel Oil Usage And Stack Effluents Summary . . . . . . . . . TRA-15 
PAGE: 1

IDAHO OPERATIONS OFFICE

$27-J U L-95$

SCHED. NO. INRPTOOBA

UNITED STATES DEPARTMENT OF ENERGY

INEL. NONRADIOLOGICAL WASTE MANAGEMENT INFORMATION SYSTEM

FOR JANUARY THROUGH DECEMBER 1994

TRA LIQUID DISPOSED SUBSTANCES SUMMARY

RPT 235-1

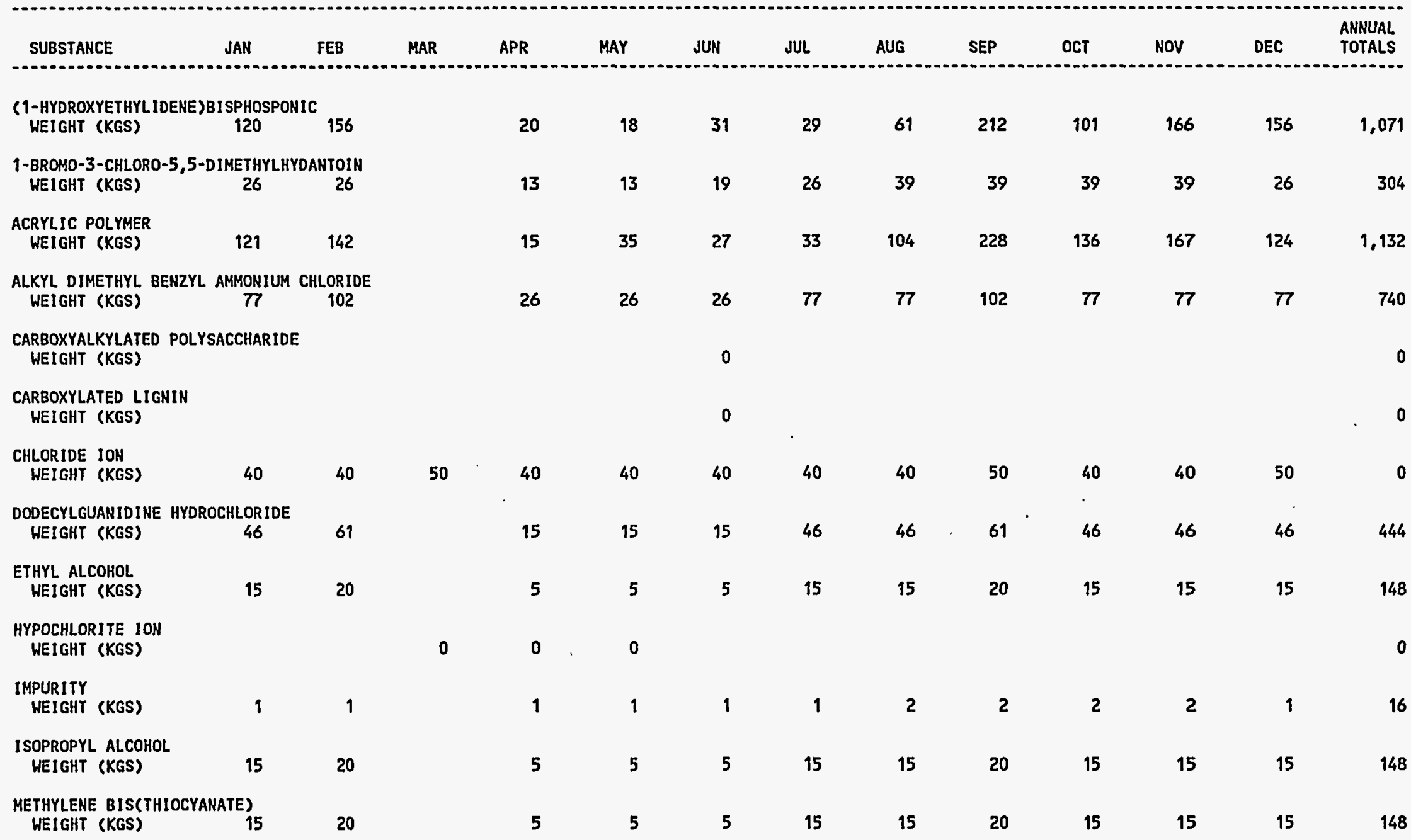

DETAILS MAY NOT ADD UP TO TOTALS BECAUSE OF ROUNDING 0 IN A COLUMN INDICATES A TOTAL OF $<0.5$ 
PAGE: 2

SCHED. NO. INRPTOOBA

IDAHO OPERATIONS OFFICE

27-JUL-95

UNITED STATES DEPARTMENT OF ENERGY

DLGICAL HASTE MANAGEMENT INFORMATION SYSTEM

INEL NONRADIOLOG SALUARY THROUGH DECEMBER 1994
FOR JANUA

TRA LIQUID DISPOSED SUBSTANCES SUMMARY

RPT 235-I

TRA

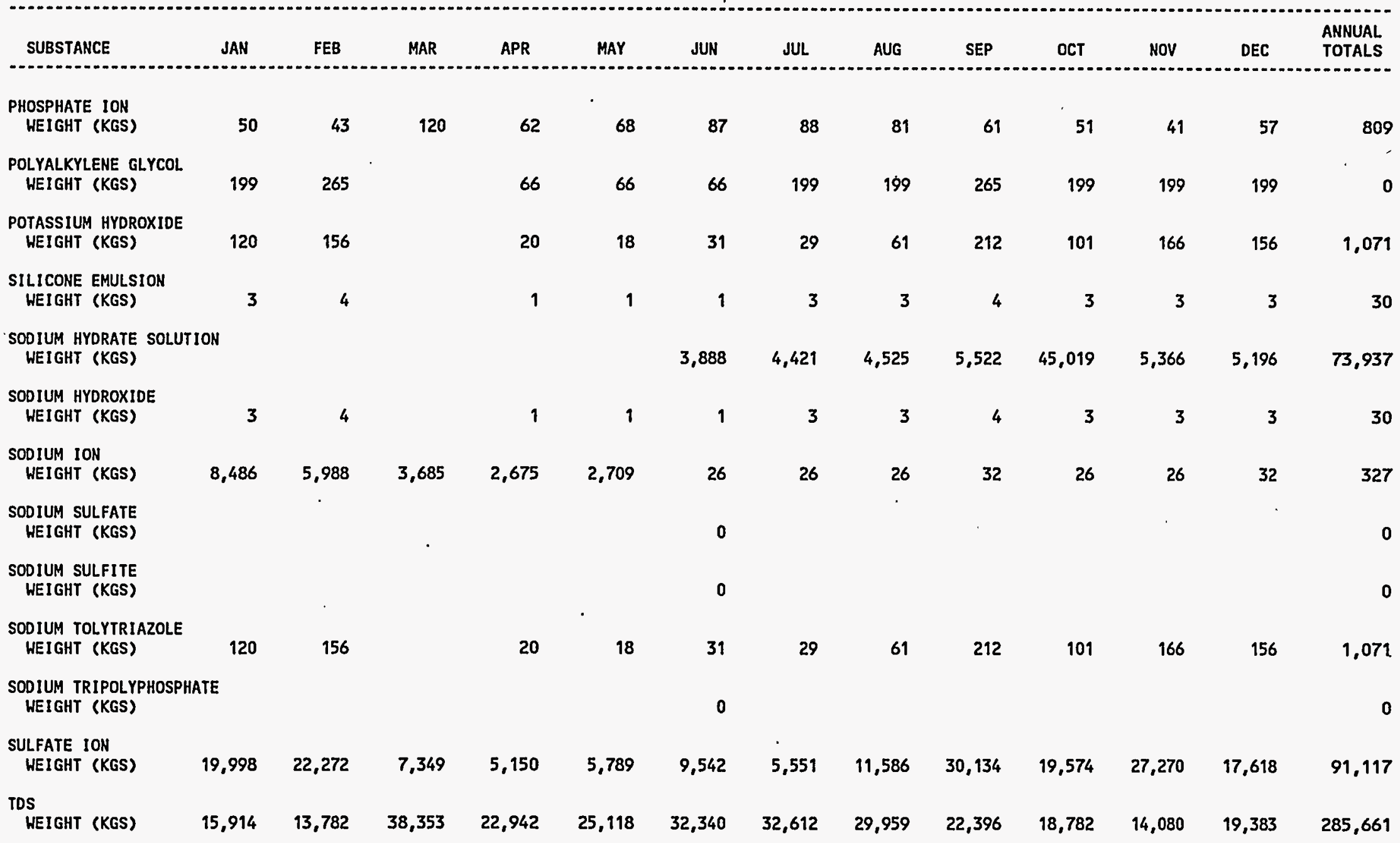

DETAILS MAY NOT ADD UP TO TOTALS BECAUSE OF ROUNDING

$O$ IN A COLUMN INDICATES A TOTAL OF $<0.5$ 
TRA LIQUID DISPOSED SUBSTANCES SUMMARY

\begin{tabular}{|c|c|c|c|c|c|c|c|c|c|c|c|c|c|}
\hline SUBSTANCE & JAN & FEB & MAR & APR & MAY & JUN & JUL. & AUG & SEP & OCT & NOV & DEC & $\begin{array}{l}\text { ANNUAL } \\
\text { TOTALS }\end{array}$ \\
\hline $\begin{array}{l}\text { TETRAPOTASSIUM } \\
\text { WEIGHT (KGS) }\end{array}$ & $\begin{array}{c}\text { HOSPHATE } \\
301\end{array}$ & 391 & & 50 & 44 & 78 & 74 & 153 & 530 & 254 & 414 & 390 & 2,677 \\
\hline $\begin{array}{l}\text { TOTAL HARDNESS } \\
\text { HEIGHT (KGS) }\end{array}$ & 10,941 & 9,475 & 26,368 & 14,261 & 15,614 & 20,103 & 20,272 & 18,623 & 13,922 & 12,284 & 9,525 & 13,112 & 184,500 \\
\hline $\begin{array}{l}\text { TRIBUTYLTIN OXI } \\
\text { WEIGHT (KGS) }\end{array}$ & 15 & 20 & & 5 & 5 & 5 & 15 & 15 & 20 & 15 & 15 & 15 & 48 \\
\hline
\end{tabular}




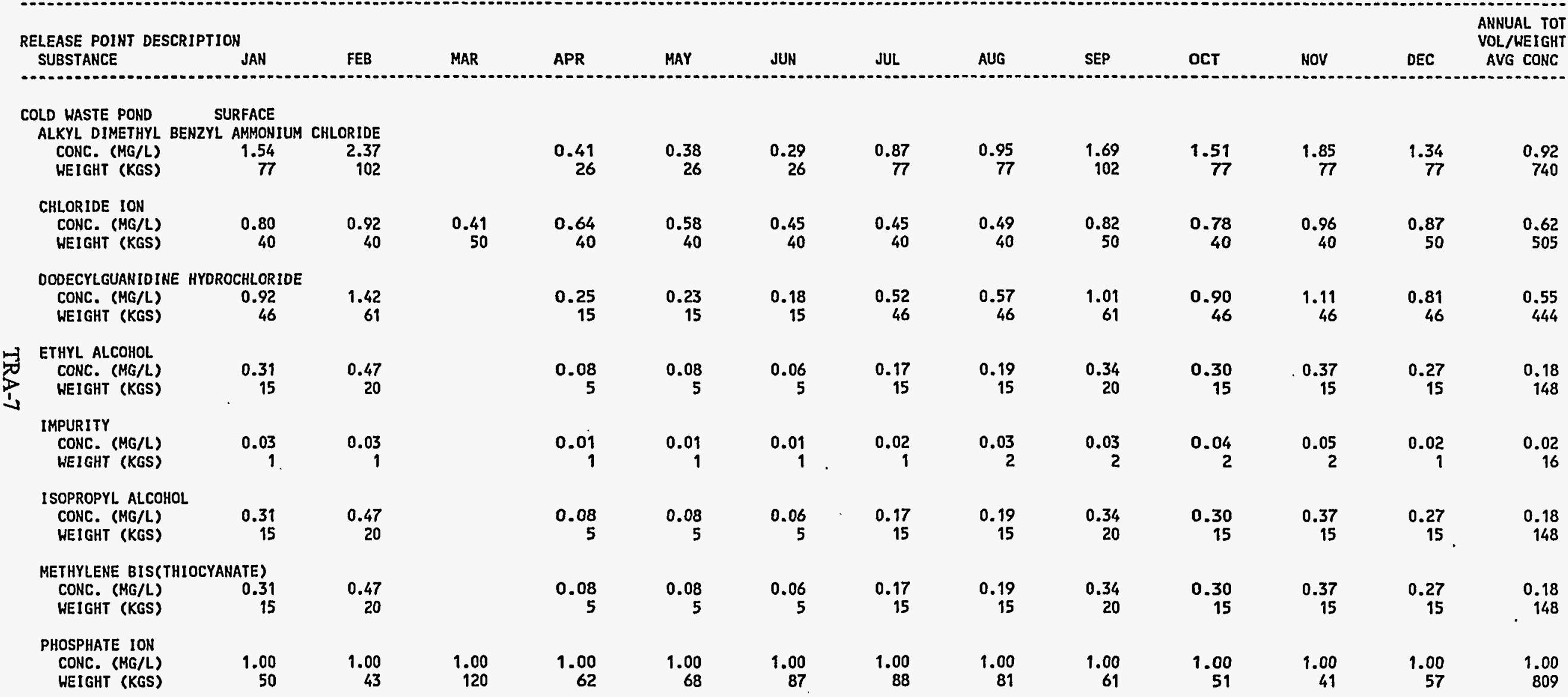


INEL NONRADIOLOGICAL HASTE MANAGEMENT INFORMATION SYSTEM

FOR JANUARY THROUGH DECEMBER 1994

TRA RELEASE POINT AND LIQUID DISPOSED SUBSTANCES SUMMARY

RPT 235-1

TRA

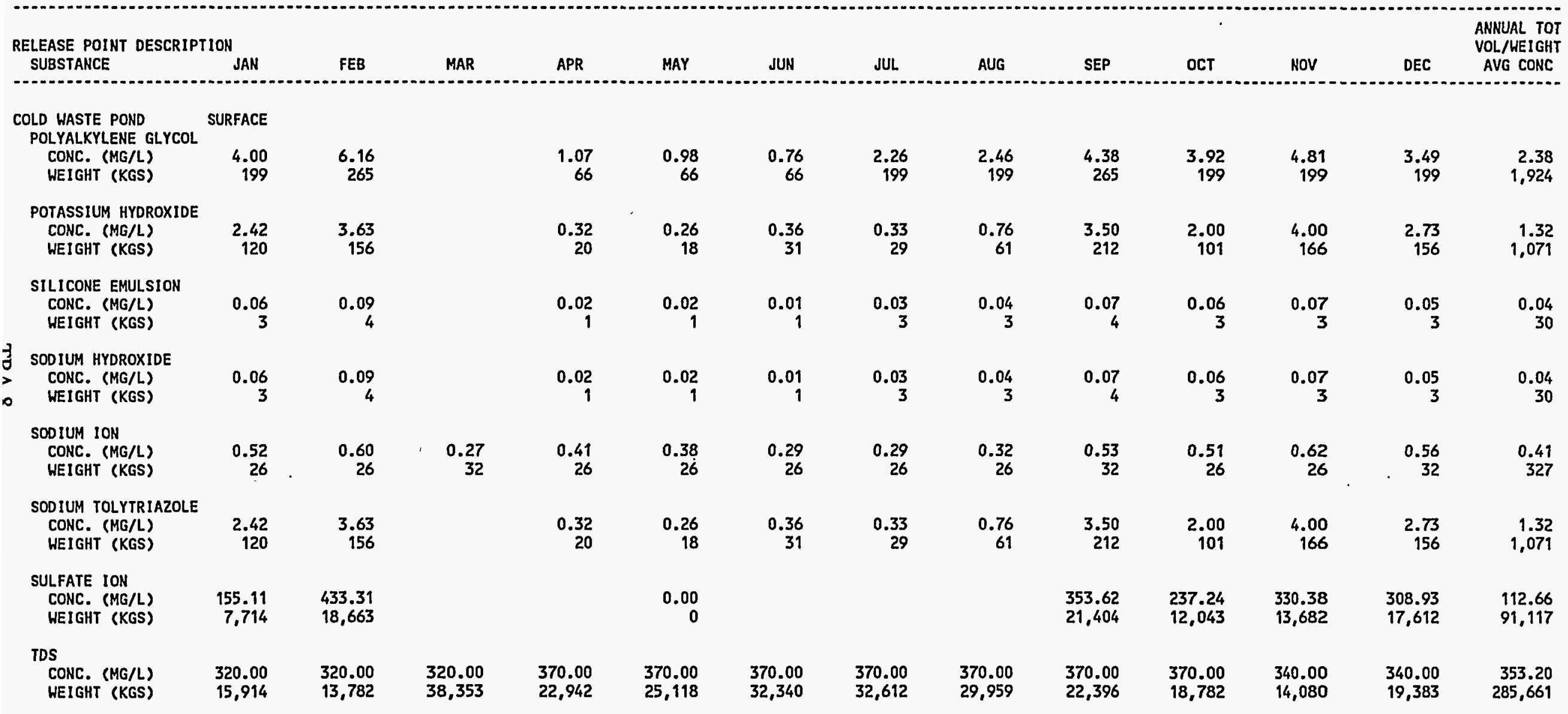




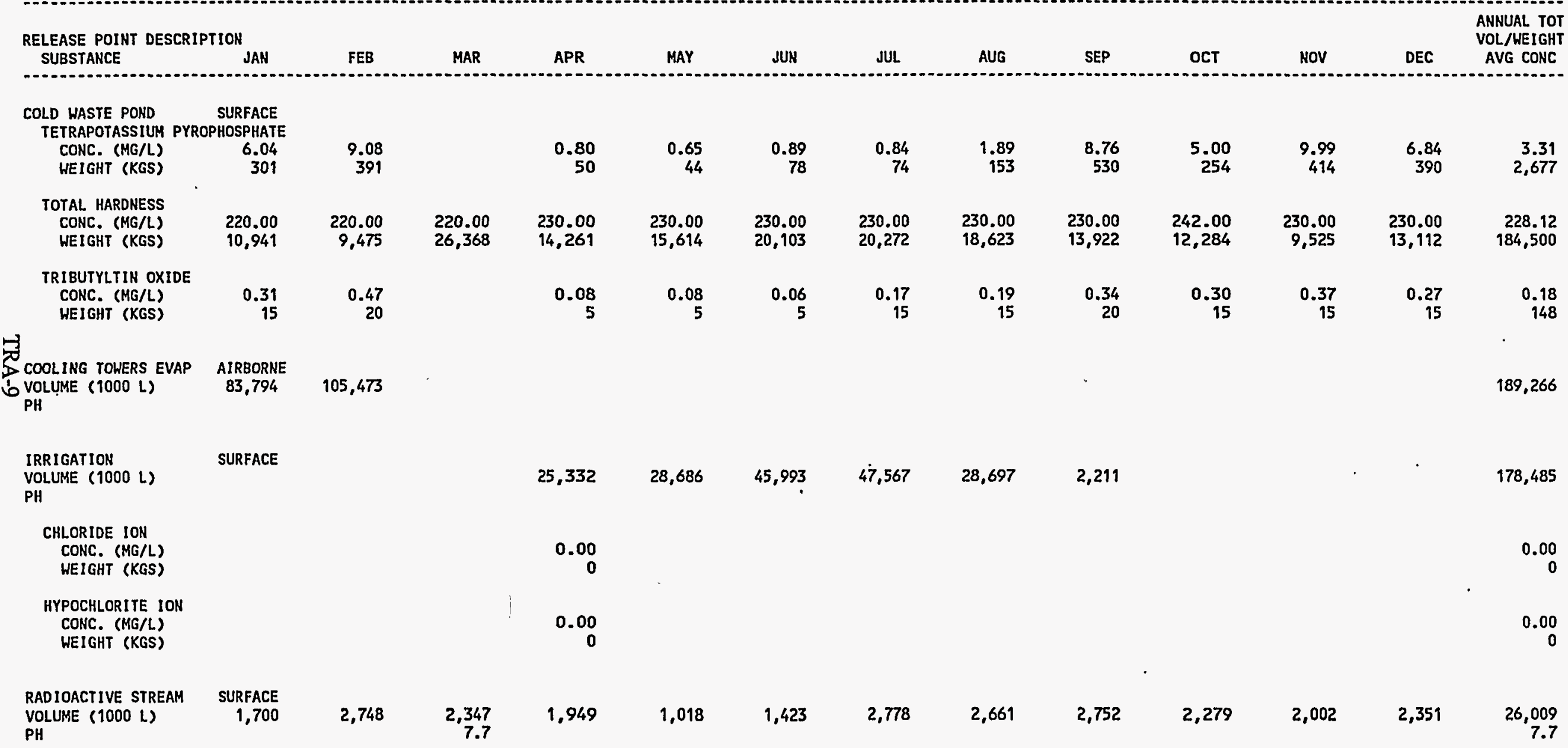

DETAILS MAY NOT ADD UP TO TOTALS BECAUSE OF ROUNDING

0 IN A COLUMN INDICATES A TOTAL OF $<0.5$

0.00 IN A COLUMN INDICATES A TOTAL OF $<0.005$ 


\begin{tabular}{|c|c|c|c|c|c|c|c|c|c|c|c|c|}
\hline $\begin{array}{l}\text { RELEASE POINT DESCRIPTION } \\
\text { SUBSTANCE }\end{array}$ & FEB & MAR & APR & MAY & JUN & JUL. & AUG & SEP & OCT & NOV & DEC & $\begin{array}{l}\text { ANNUAL TOT } \\
\text { VOL/WEIGHT } \\
\text { AVG CONC }\end{array}$ \\
\hline $\begin{array}{l}\text { RADIOACTIVE STREAM } \\
\text { CHLORIDE ION } \\
\text { CONC. (MG/L) } \\
\text { WEIGHT (KGS) }\end{array}$ & & $\begin{array}{r}0.00 \\
0\end{array}$ & $\begin{array}{r}0.00 \\
0\end{array}$ & $\begin{array}{r}10.00 \\
0\end{array}$ & & & & & & & & $\begin{array}{r}0.00 \\
0\end{array}$ \\
\hline $\begin{array}{l}\text { HYPOCHLORITE ION } \\
\text { CONC. (MG/L) } \\
\text { WEIGHT (KGS) }\end{array}$ & & $\begin{array}{r}0.00 \\
0\end{array}$ & $\begin{array}{r}0.00 \\
0\end{array}$ & $\begin{array}{r}0.00 \\
0\end{array}$ & & & & & & & & $\begin{array}{r}0.00 \\
0\end{array}$ \\
\hline $\begin{array}{lr}\text { SEWAGE PLANT } & \text { SURFACE } \\
\text { VOLUME }(1000 \mathrm{~L}) & 5,148 \\
\text { PH } & \end{array}$ & 4,766 & $\begin{array}{r}4.732 \\
7.7\end{array}$ & $\begin{array}{r}5.663 \\
7.3\end{array}$ & $\begin{array}{r}5,004 \\
7.4\end{array}$ & 4,997 & 6,193 & $\begin{array}{r}6,617 \\
7.6\end{array}$ & 7,366 & 7,393 & 62,762 & 5,758 & $\begin{array}{r}126,398 \\
7.5\end{array}$ \\
\hline $\begin{array}{l}\text { CARBOXYALKYLATED POLYSACCHAR } \\
\text { CONC. (MG/L) } \\
\text { WEIGHT (KGS) }\end{array}$ & & & & . & $\begin{array}{r}0.00 \\
0\end{array}$ & & & & & & & $\begin{array}{r}0.00 \\
0\end{array}$ \\
\hline $\begin{array}{l}\text { CARBOXYLATED LIGNIN } \\
\text { CONC. (MG/L) } \\
\text { WEIGHT (KGS) }\end{array}$ & & & & & $\begin{array}{r}0.00 \\
0\end{array}$ & . & & & & & & $\begin{array}{r}0.00 \\
0\end{array}$ \\
\hline $\begin{array}{l}\text { CHLORIDE ION } \\
\text { CONC. (MG/L) } \\
\text { WEIGHT (KGS) }\end{array}$ & & $\begin{array}{r}0.00 \\
0\end{array}$ & $\begin{array}{r}0.00 \\
0\end{array}$ & $\begin{array}{r}0.00 \\
0\end{array}$ & & & & v & & & & $\begin{array}{r}0.00 \\
0\end{array}$ \\
\hline $\begin{array}{l}\text { HYPOCHLORITE ION } \\
\text { CONC. (MG/L) } \\
\text { WEIGHT (KGS) }\end{array}$ & & $\begin{array}{r}0.00 \\
0\end{array}$ & $\begin{array}{r}0.00 \\
0\end{array}$ & $\begin{array}{r}0.00 \\
0\end{array}$ & & & & & & & & $\begin{array}{r}0.00 \\
0\end{array}$ \\
\hline $\begin{array}{l}\text { POLYALKYLENE GLYCOL } \\
\text { CONC. (MG/L) } \\
\text { WEIGHT (KGS) }\end{array}$ & & & & & $\begin{array}{r}0.00 \\
0\end{array}$ & & & & & & & $\begin{array}{r}0.00 \\
0\end{array}$ \\
\hline
\end{tabular}


PAGE: 6

SCHED. NO. INRPTOOB
IDAHO OPERATIONS OFFICE

27-JUL-95

WLGICAL HASTE MANAGEMEHT INFORMATION SYSTEM

FOR JANUARY THROUGH DECEMBER 1994

TRA RELEASE POINT AND LIQUID DISPOSED SUBSTANCES SUMMARY

RPT 235-1

TRA

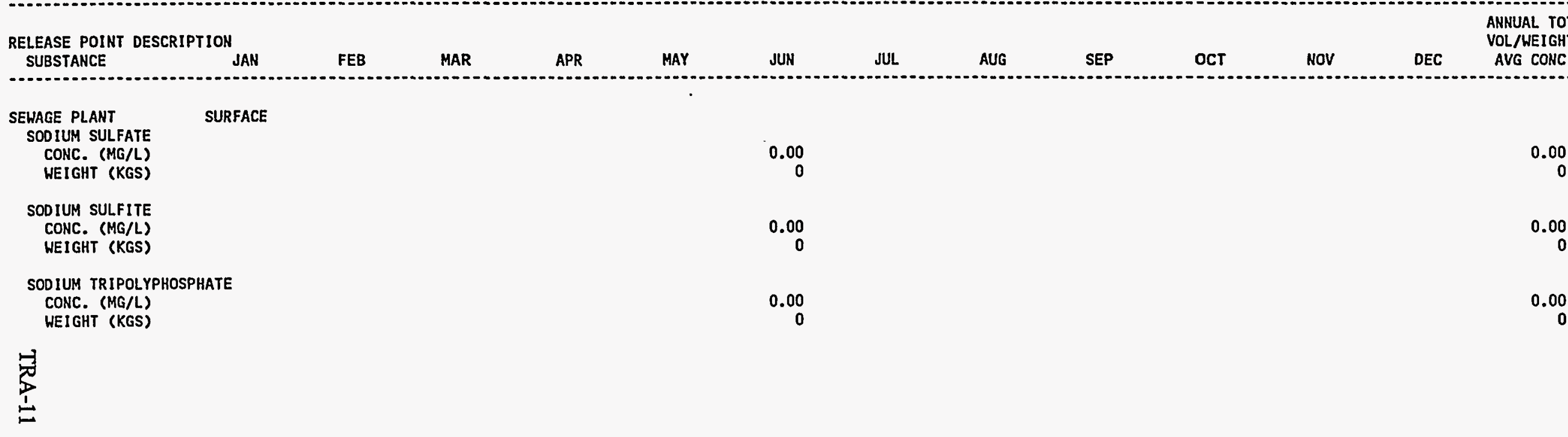


PAGE: 1

SCHED. NO. INRPTOOS

IDAHO OPERATIONS OFFICE

27-JUL-95

UNITED STATES DEPARTMENT OF ENERGY

INEL NONRADIOLOGICAL. WASTE MANAGEMENT INFORMATION SYSTEM

FOR JANUARY THROUGH DECEMBER 1994

TRA LANDFILL SOLID DISPOSED WASTE SUMMARY

RPT 236-I

TRA

(ALL VOLUMES = CUBIC METERS)

\begin{tabular}{|c|c|c|c|c|c|c|c|c|c|c|c|c|c|}
\hline Type of Waste & JAN & FEB & MAR & APR & MAY & JUN & JUL & AUG & SEP & OCT & NOV & DEC & TOTALS \\
\hline $\begin{array}{l}\text { ASBESTOS } \\
\text { ASPHALT } \\
\text { DIRT AND GRAVEL } \\
\text { MASONRY AND CONCRETE } \\
\text { SCRAP METAL } \\
\text { TRASH, SWEEPINGS, ETC. } \\
\text { WEEDS, GRASS AND TREES } \\
\text { WOOD AND SCRAP LUMBER }\end{array}$ & $\begin{array}{r}3.1 \\
186.9 \\
6.1\end{array}$ & $\begin{array}{r}9.2 \\
226.7 \\
3.8\end{array}$ & $\begin{array}{r}9.2 \\
300.9\end{array}$ & $\begin{array}{r}9.6 \\
278.3 \\
22.9\end{array}$ & 182.7 & $\begin{array}{r}29.1 \\
1.5 \\
3.4 \\
86.0 \\
147.2 \\
87.9\end{array}$ & $\begin{array}{r}9.9 \\
9.2 \\
116.2\end{array}$ & $\begin{array}{r}2.7 \\
13.8 \\
196.5 \\
1.5\end{array}$ & $\begin{array}{r}1.1 \\
7.6 \\
114.7 \\
0.8 \\
9.2\end{array}$ & $\begin{array}{r}3.1 \\
105.5 \\
13.0\end{array}$ & $\begin{array}{r}6.1 \\
120.8 \\
7.6\end{array}$ & 85.6 & $\begin{array}{r}29.1 \\
1.1 \\
1.5 \\
20.3 \\
153.7 \\
2.062 .1 \\
0.8 \\
152.2\end{array}$ \\
\hline 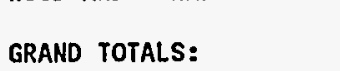 & 196.1 & 239.7 & 310.0 & 310.8 & 182.7 & 355.2 & 135.3 & 214.5 & 133.4 & 122.7 & 134.6 & & $2,420.7$ \\
\hline
\end{tabular}

葲 
FOR JANUARY THROUGH DECEMBER 1994

TRA HATER USAGE AND D́ISPOSAL SUMMARY

RPT 237-I

TRA

(ALL VOLUMES = NEAREST THOUSAND LITERS)

ANNUAL TOT HELL OR BLDG. NO./ JAN FEB MAR APR MAY JUN

JUL AUG SEP OCT NOV DEC AVG CONC

\section{HATER DATA} 001 WATER PUMPED (L)
004 HATER PUMPED (L)

$\begin{array}{llrr}\text { (L) } & & & 4,709 \\ \text { (L) } & 142,255 & 158,305 & 124,422\end{array}$ WATER PUMPED (L) WATER DISPOSED AIR (L) SURFACE (L) SUBSURFACE (L)

158,305
142,255

29,131

96,717

$104,212 \quad 137,031 \quad 146,419-151$

$141,953 \quad 160,690 \quad 175,264,1,744,460$ 96,717 104,212 137,03 146,419 151,037 05,926

$\begin{array}{llll}161,953 \quad 160,690 & 175,264 & 1,744,233\end{array}$ SWAGE DATA

BUILD VOL (L)

$$
5,148 \quad 4,766
$$

4,732

5,663

$$
500
$$

BIOCHEMICAL OXYGEN DEMAND (BOD)

RAH CONC.

76.0

121.3
28.0

87.5
21.0

71.0
25.2

133.8
25.3
81.1

136,53

14.478

151,037

130,748
67,834

157,704

160,690

$175,264 \quad 1,764,693$

$\%$ REMOVED

72.9

76.9

76.0

64.5

DISSOLVED OXYGEN (DO)

RAW CONC. (MG/L)

FINAL CONC. (MG/L)

INCPEASED PACTOR

5.7

4.4
5.5
0.3

4.4
5.4

SETTLEABLE SOLIDS (SS)

RAW CONC. (ML/L)
FINAL CONC. (ML/L)
\% REMOVED

2.6
0.11

6.1

95.8

$\begin{array}{rr}6.1 & 4.2 \\ 0.14 & 0.11 \\ 97.7 & 97.4\end{array}$

PH FIMAL

$\begin{array}{lll}7.3 & 7.5 & 7.7\end{array}$

TOTAL SEHAGE(L)

$5,148 \quad 4,766 \quad 4,732$

TOTAL HATER DISPOSED(L) $142,267 \quad 158,321 \quad 129,131$

$$
3.7
$$

2.8
0.08
97.

$3.2 \quad 3.1 \quad 2.4$

7.3
5,663

96,718

\section{$\begin{array}{lll}3.1 & 2.4 & 2.4 \\ 5.7 & 4.3 & 5.6\end{array}$}

5.7
0.8

2.4
4.3
0.8

$\begin{array}{rrr}4.6 & 3.8 & 4.3 \\ 0.17 & 0.21 & 0.18 \\ 96.3 & 94.5 & 95.8 \\ 7.4 & 7.2 & 7.3 \\ 500 & 4.997 & 6.193\end{array}$
DETAILS MAY NOT ADD UP TO TOTALS BECAUSE OF ROUNDING
0 IN A COLUMN INDICATES A TOTAL OF $<0.5$

0.0 IN A COLUMN INDICATES A TOTAL OF $<0.05$

0.00 IN A COLUMN INDICATES A TOTAL OF $<0.005$ 
PAGE: 2

SCHED. NO. INRPTO1O
IDAHO OPERATIONS OFFICE

UNITED STATES DEPARTMENT OF ENERGY

$27-J U L-95$

INEL NONRADIOLOGICAL WASTE MANAGEMENT INFORMATION SYSTEM

FOR JANUARY THROUGH DECEMBER 1994

TRA WATER USAGE AND DISPOSAL SUMMARY

(ALL VOLUMES $=$ NEAREST THOUSAND LITERS)

RPT 237-1

TRA

\begin{tabular}{|c|c|c|c|c|c|c|c|c|c|c|c|c|}
\hline WELLL OR BLDG. NO./ & & & & & & & & & & & & $\begin{array}{l}\text { ANNUAL TOT } \\
\text { VOL/HEIGH }\end{array}$ \\
\hline SEHAGE BLUG. NO. & JAN & FEB & MAR & APR & MAY & JUN & JUL & AUG & SEP & OCT & NOV & DEC AVG CONC \\
\hline CCOUNTABILITY (\%) & 100.0 & 100.0 & 100.0 & 100.0 & 95.7 & 104.3 & 101.9 & 100.0 & 100.0 & 100.0 & 100.0 & 97.3 \\
\hline
\end{tabular}


PAGE: 1
SCHED. NO. INRPT011

IDAHO OPERATIONS OFFICE

$27-$ JUL -95

UNITED STATES DEPARTMENT OF ENERGY

INEL NONRADIOLOGICAL WASTE MANAGEMENT INFORMATION SYSTEM

FOR JANUARY THROUGH DECEMBER 1994

TRA FUEL OIL USAGE AND STACK EFFLUENTS SUMMARY

RPT 238-1

TRA

(ALL VALUES = MEAREST THOUSAND)

\begin{tabular}{|c|c|c|c|c|c|c|c|c|c|c|c|c|c|}
\hline $\begin{array}{l}\text { FUEL OIL TYPE } \\
\text { EFFLUENTS }\end{array}$ & JAN & FEB & MAR & APR & MAY & JUN & JUL & AUG & SEP & OCT & NOV & DEC & $\begin{array}{l}\text { ANNUAL } \\
\text { TOTALS }\end{array}$ \\
\hline $\begin{array}{l}\text { TYPE } 2 \\
\text { VOLUME (LITERS) } \\
\text { SO2 (KGS) } \\
\text { NOX (KGS) } \\
\text { PARTICULATES (KGS }\end{array}$ & $\begin{array}{r}108 \\
1\end{array}$ & $\begin{array}{r}122 \\
1\end{array}$ & $\begin{array}{l}6 \\
0\end{array}$ & $\begin{array}{l}2 \\
0\end{array}$ & & $\begin{array}{r}18 \\
0\end{array}$ & $\begin{array}{r}68 \\
0\end{array}$ & $\begin{array}{r}100 \\
1\end{array}$ & $\begin{array}{r}169 \\
1\end{array}$ & $\begin{array}{r}106 \\
1\end{array}$ & $\begin{array}{r}123 \\
1\end{array}$ & $\begin{array}{r}123 \\
1\end{array}$ & $\begin{array}{r}945 \\
7\end{array}$ \\
\hline $\begin{array}{l}\text { GRAND TOTALS: } \\
\text { VOLUME (LITERS) } \\
\text { SO2 (KGS) } \\
\text { NOX (KGS) }\end{array}$ & $\begin{array}{r}108 \\
1\end{array}$ & $\begin{array}{r}122 \\
1\end{array}$ & $\begin{array}{l}6 \\
0\end{array}$ & $\begin{array}{l}2 \\
0\end{array}$ & & $\begin{array}{r}18 \\
0\end{array}$ & $\begin{array}{r}68 \\
0\end{array}$ & $\begin{array}{r}900 \\
1\end{array}$ & $\begin{array}{r}169 \\
1\end{array}$ & $\begin{array}{r}106 \\
1\end{array}$ & $\begin{array}{r}123 \\
1\end{array}$ & $\begin{array}{r}123 \\
1\end{array}$ & 945 \\
\hline
\end{tabular}


- 
Technical Support Facility-Test Area North 1994 Detail Data

Report 225-TSF Liquid Disposed Substances Summary . . . . . . . . . . TSF-3

Report 225-TSF Release Point And Liquid Disposed Substances Summary . . . . T TSF-4

Report 227-TSF Water Usage And Disposal Summary . . . . . . . . . . TSF-6

Report 228-TSF Fuel Oil Usage And Stack Effluents Summary . . . . . . . . . TSF-8 

PAGE: 1

SCHED. NO. INRPTOOBA

\begin{tabular}{|c|c|c|c|c|c|c|c|c|c|c|c|c|c|}
\hline SUBSTANCE & JAN & FEB & MAR & APR & MAY & JUN & JUL & AUG & SEP & OCT & Nov & DEC & $\begin{array}{l}\text { ANNUAL } \\
\text { JOTALS }\end{array}$ \\
\hline $\begin{array}{cl}\text { CHLORIDE } & \text { ION } \\
\text { WEIGHT } & \text { (KGS) }\end{array}$ & 619 & 303 & 179 & 275 & 96 & & & & & 193 & 179 & 578 & 2,422 \\
\hline $\begin{array}{l}\text { CYCLOHEXYLAMINE } \\
\text { WEIGHT (KGS) }\end{array}$ & 1 & 3 & 0 & 1 & 0 & & & & & 1 & 0 & 1 & 7 \\
\hline $\begin{array}{l}\text { EDETATE SODIUM } \\
\text { HEIGHT (KGS) }\end{array}$ & & & & & & & & & & 16 & 6 & 6 & 27 \\
\hline $\begin{array}{l}\text { ETHYLENED IAMINE TETRAA } \\
\text { HEIGHT (KGS) }\end{array}$ & $\underset{9}{\text { ICETIC ACID }}$ & 12 & 1 & 12 & 2 & & & & & & & & 36 \\
\hline $\begin{array}{l}\text { JANITORIAL SUPPLIES } \\
\text { HEIGHT (KGS) }\end{array}$ & 277 & 82 & & & & & & & & & & & 359 \\
\hline $\begin{array}{l}\text { LIGNOSULFONIC ACID, SO } \\
\text { HEIGHT (KGS) }\end{array}$ & DIUM SALT & & & & & & & & & 16 & 6 & 6 & 27 \\
\hline $\begin{array}{l}\text { MORPHOL INE } \\
\text { HEIGHT (KGS) }\end{array}$ & 1 & 3 & 0 & 1 & 0 & & & & & 1 & 0 & 1 & 7 \\
\hline $\begin{array}{l}\text { POTASSIUM HYDROXIDE } \\
\text { HEIGHT (KGS) }\end{array}$ & 9 & 12 & 1 & 12 & 2 & & & & & & & ${ }^{\circ}$ & 36 \\
\hline $\begin{array}{l}\text { SOOIUM ION } \\
\text { HEIGHT (KGS) }\end{array}$ & 415 & 211 & 123 & 178 & 62 & & & & & 125 & 124 & 380 & 1,620 \\
\hline $\begin{array}{l}\text { SODIUM LIGNOSULFONATE } \\
\text { HEIGHT (KGS) }\end{array}$ & 9 & 12 & 1 & 12 & 2 & & & & & & & & 36 . \\
\hline $\begin{array}{l}\text { SULFATE ION } \\
\text { HEIGHT (KGS) }\end{array}$ & & & 13 & & & & & & & & & & 13 \\
\hline $\begin{array}{l}\text { SULFITE ION } \\
\text { HEIGHT (KGS) }\end{array}$ & 24 & 26 & & & & & & & & & 14 & 10 & 75 \\
\hline
\end{tabular}


FOR JANUARY THROUGH DECEMBER 1994

TSF RELEASE POINT AND LIQUID DISPOSED SUBSTANCES SUMMARY

RPT 225-1

TSF

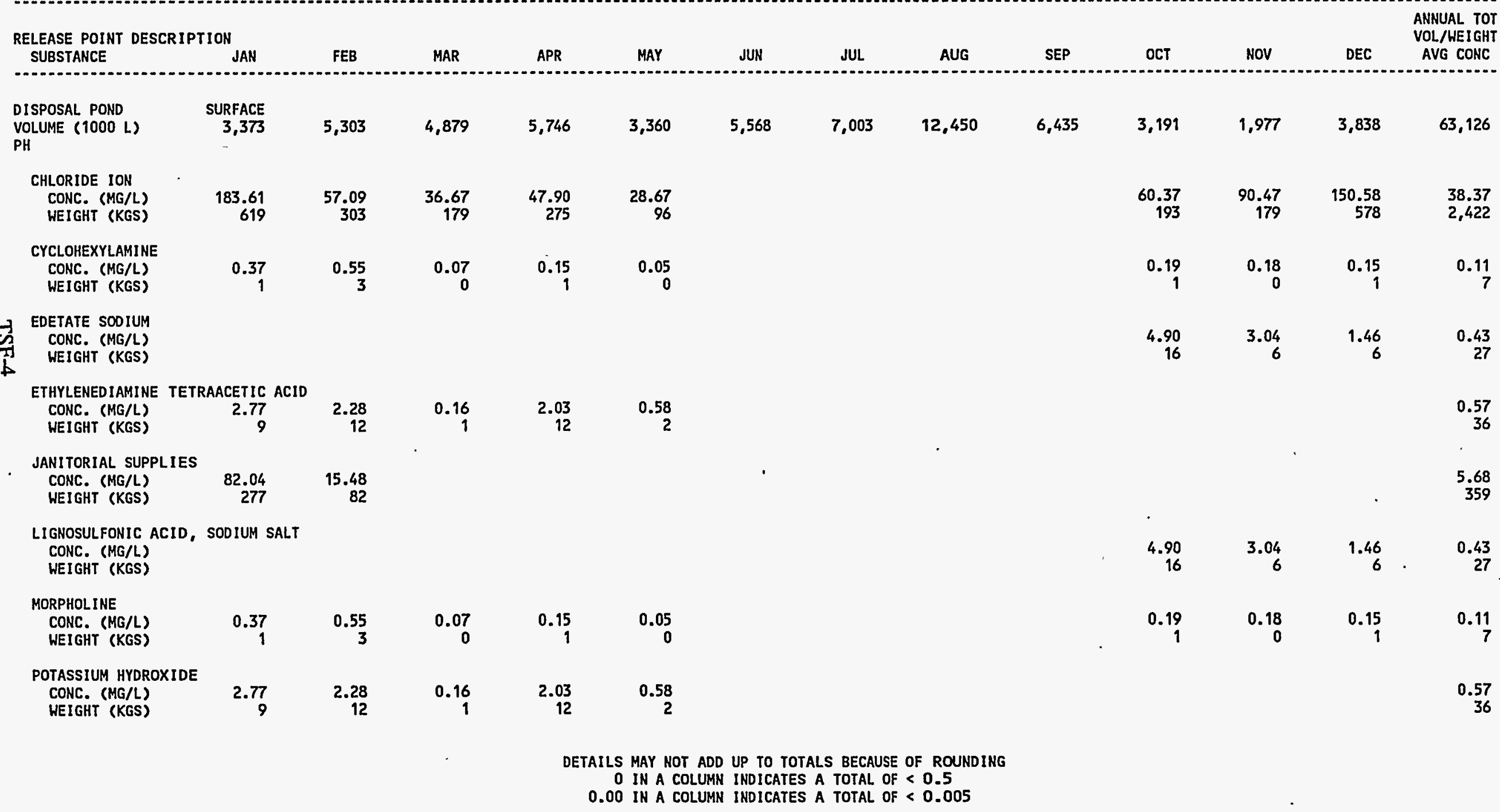


PAGE: 2

SCHED. NO. INRPTOO8
IDAHO OPERATIONS OFFICE

27-JUL-95

UNITED STATES DEPARTMENT OF ENERGY

INEL NONRADIOLOGICAL YASTE MANAGEMENT INFORMATION SYSTEM

FOR JANUARY THROUGH DECEMBER 1994

TSF RELEASE POINT AND LIOUID DISPOSED SUBSTANCES SUMMARY

RPT 225-I

TSF

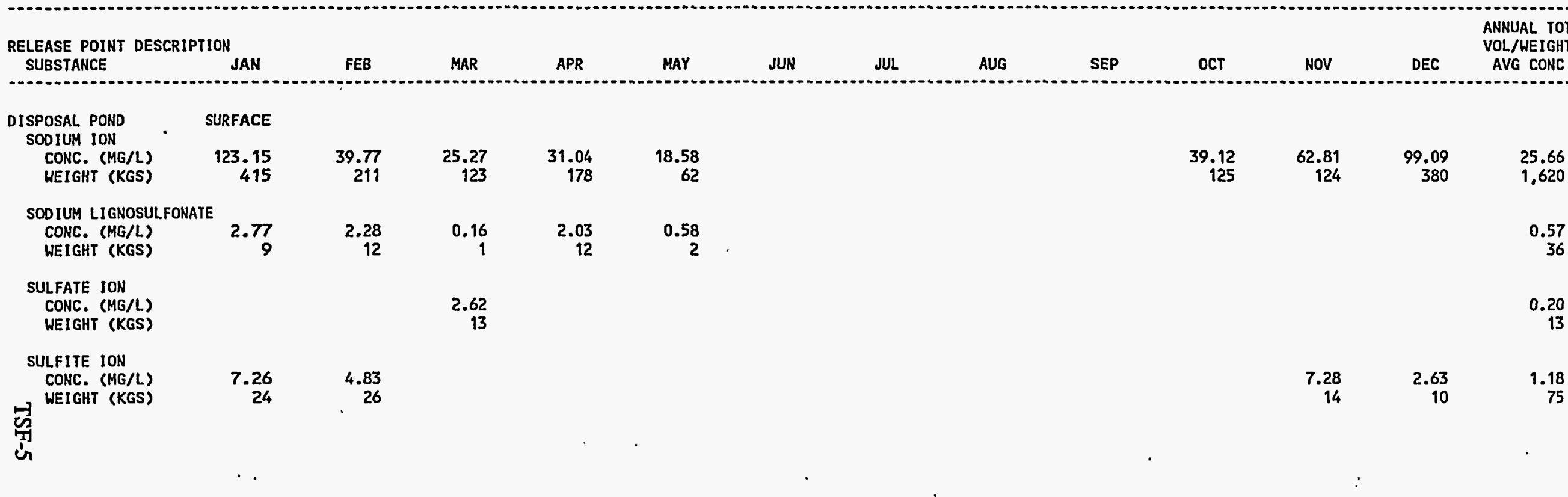

DETAILS MAY NOT ADD UP TO TOTALS'BECAUSE OF ROUNDING

$O$ IN A COLUMN INDICATES A TOTAL OF $<0.5$

0.00 IN A COLUMN INDICATES A TOTAL OF $<0.005$ 
PAGE: 11
SCHED. NO. INRPT010

IDAHO OPERATIONS OFFICE

27-JUL-95

UNITED STATES DEPARTMENT OF ENERGY

INEL NONRADIOLOGICAL HASTE MANAGEMENT INFORMATION SYSTEM

FOR JANUARY THROUGH DECEMBER 1994

TSF WATER USAGE AND DISPOSAL SUMMARY

RPT 227-1

TSF

(ALL VOLUMES = NEAREST THOUSAND LITERS)

ANNUAL TOT
VOL/WEIGHT

WELL OR BLDG. NO./

JAN FEB MAR APR MAY JUN JUL AUG

SEP

OCT

NOV DEC AVG CONC

WATER DATA

612 WATER PUMPED (L)

613 HATER PUMPED (L)

HATER PUMPED (L)

\begin{tabular}{rr}
3,706 & 4,243 \\
3,706 & 4,56 \\
\hline 7,412 & 8,812
\end{tabular}

3,888

WATER DISPOSED

AIR (L)

SURFACE (L)

3,373

5,303

4,879

5,746

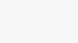

SEWAGE DATA

623 BUILD VOL (L)

$4,039 \quad 3,508 \quad 3,065$

3,028

$3,173 \quad 3,092$

$3,223 \quad 4,300$

443

3,895

3,737

3,232

38,735

BIOCHEMICAL OXYGEN DEMAND (BOD)

$\begin{array}{lcl}\text { RAH CONC. (MG/L) } & 70.0 & 37.0\end{array}$

FINAL CONC.

$\begin{array}{lr}10.0 & 6.3 \\ 85.7 & 83.0\end{array}$

$80.8 \quad 69.0$

\section{3}

\% REMOVED

8.4
89.6

86.5

38.3
11.8
69.2

40.4

. 44.0

41.5

39.

DISSOLVED OXYGEN (DO)

RAW CONC. (MG/L)

FINAL CONC. (MG/L)

$\begin{array}{ll}7.2 & 6.8 \\ 7.7 & 7\end{array}$

INCREASED FACTOR

6.8
7.7

4.9
5.9
0.2

6.7

$\begin{array}{llll}4.0 & 3.7 & 4.0 & 3.8 \\ 5.7 & 5.4 & 5.2 & 5.0\end{array}$

77.0

49.
12.8
74

42.8
74.1

35.3
8.3

8.3
76.5

$44.2 \quad 49.1$

SETTLEABLE SOLIDS (SS)

RAH CONC. (ML/L)
FINAL CONC. (ML/L)

5.36 .6

$\%$ REMOVED

5.8
0.00

0.00
100.0

$$
\begin{array}{r}
5.8 \\
99.7
\end{array}
$$

$\begin{array}{rrrr}5.8 & 4.6 & 4.1 & 1.8\end{array}$

PH FINAL

$8.0 \quad 8.3$

7.9

99.3

1.8
0.01

99.4

4.5
0.03

0.03
99.3

3.7
5.1

4.7

5.9

3.9

84.6

81.0

TOTAL SEHAGE(L)

$4,039 \quad 3,508$

3,065

3,028

7.4

7.4

7.6

7.4

3.2
0.02

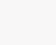

0.8

$6.3 \quad 4.9$

TOTAL WATER DISPOSED(L)

$8,811 \quad 7,944$

8,775

$3,173 \quad 3,092 \quad 3,223$

4,300

99.4

4.9
0.00
100.0

2.3
0.00

0.00
100.0

$\begin{array}{ll}3.2 & 4.3\end{array}$

$\begin{array}{ll}0.02 & 0.02 \\ 99.4 & 99.6\end{array}$

7.6

$7.9 \quad 7.9$

$8.0 \quad 7.8$

DETAILS MAY NOT ADD UP TO TOTALS BECAUSE OF ROUNDING

0 IN A COLUMN INDICATES A TOTAL OF $<0.5$

0.0 IN A COLUMN INDICATES A TOTAL OF $<0.05$

0.00 IN A COLUMN INDICATES A TOTAL OF $<0.005$ 
PAGE: 2

SCHED. NO. INRPTOIO
IDAHO OPERATIONS OFFICE

UHITED STATES DEPARTUENT OF ENERGY

$27-\mathrm{JUL}-95$

INEL NONRADIOLOGICAL WASTE MANAGEMENT INFORMATION SYSTEM

FOR JANUARY THROUGH DECENBER 1994

TSF HATER USAGE AND DISPOSAL. SUMMARY

(ALL VOLUMES $=$ NEAREST THOUSAND LITERS)

RPT 227-I

TSF

ANNUAL TOT
VOL/WEIGHT

SEWAGE BLDG. NO. JAN FEB MAR APR MAY JUN JUL AUG SEP OCT NOV DEC AVG CONC

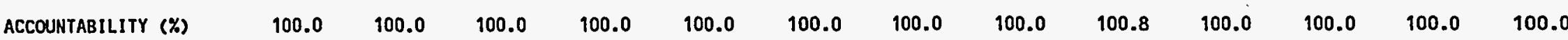

0 IN A COLUMN INDICATES A TOTAL OF $<0.5$

0.0 IN A COLUMA INDICATES A TOTAL OF $<0.05$

0.00 IN A COLUMN INDICATES A TOTAL OF $<0.005$ 
PAGE: 1

SCHED. NO. INRPT011
IDAHO OPERATIONS OFFICE

27-JUL-95

UNITED STATES DEPARTMENT OF ENERGY

INEL NONRADIOLOGICAL WASTE MANAGEMENT INFORMATION SYSTEM

FOR JANUARY THROUGH DECEMBER 1994

TSF FUEL OIL USAGE AND STACK EFFLUENTS SUMMARY

RPT 228-1

TS

(ALL VALUES $=$ NEAREST THOUSAND)

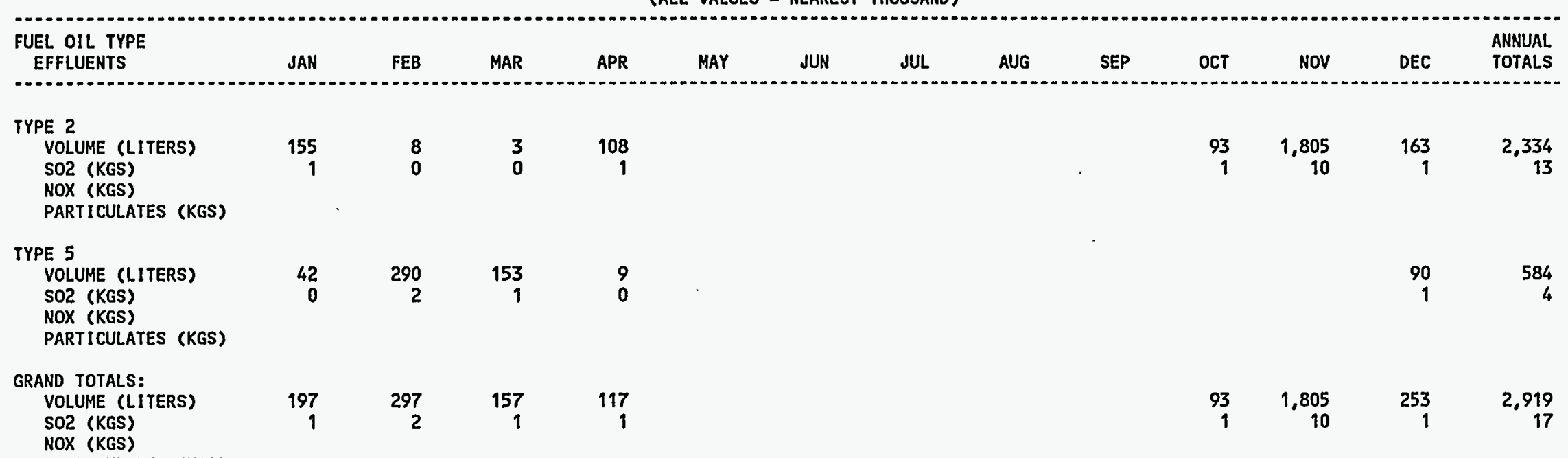

$O$ IN A COLUMN INDICATES A TOTAL OF $<0.5$ 
Water Reactor Research Test Facility-Test Area North 1994 Detail Data

Report 265-WRF Liquid Disposed Substances Summary . . . . . . . . . . . WRF-3 Report 265-WRF Release Point And Liquid Disposed Substances Summary . . . . . WRF-4 Report 267-WRF Water Usage And Disposal Summary . . . . . . . . . . W WRF-6 Report 268-WRF Fuel Oil Usage And Stack Effluents Summary . . . . . . . . . WRF-8 


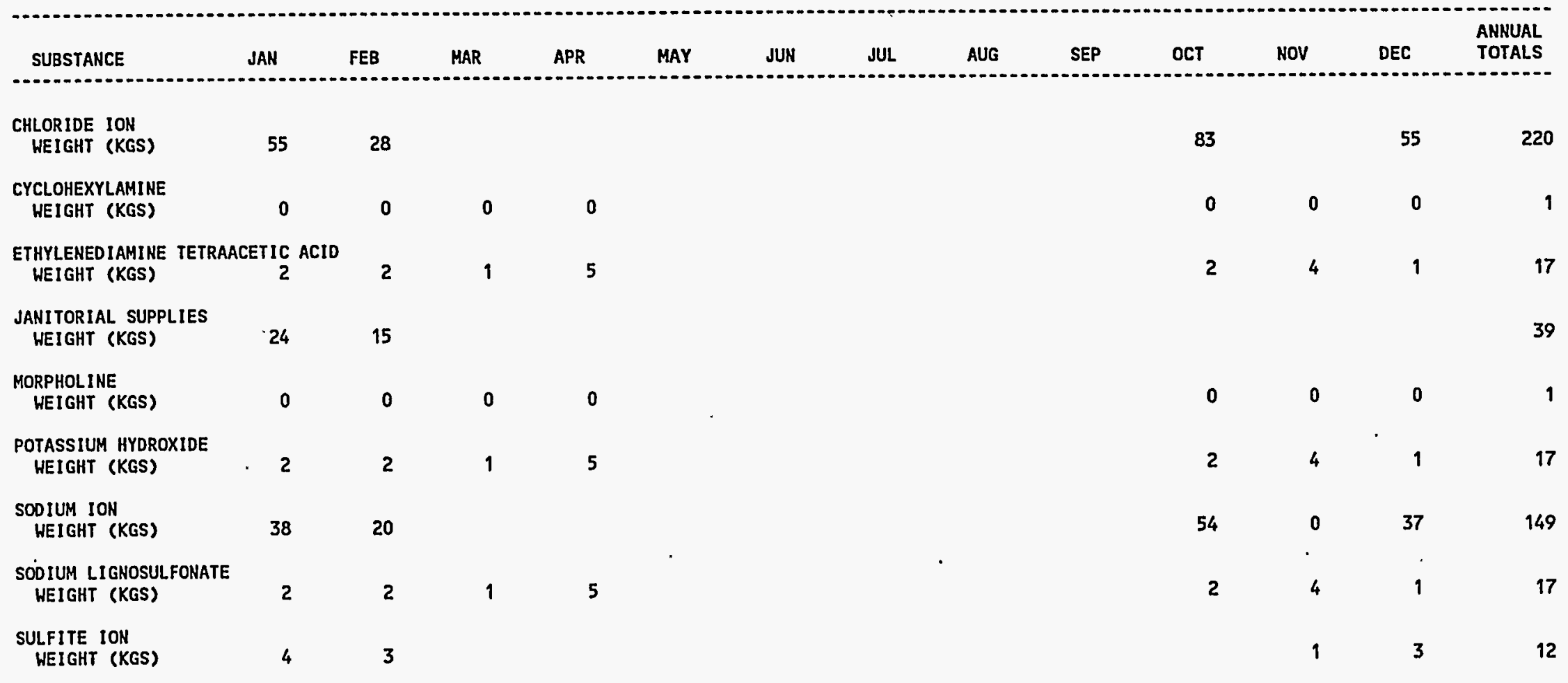




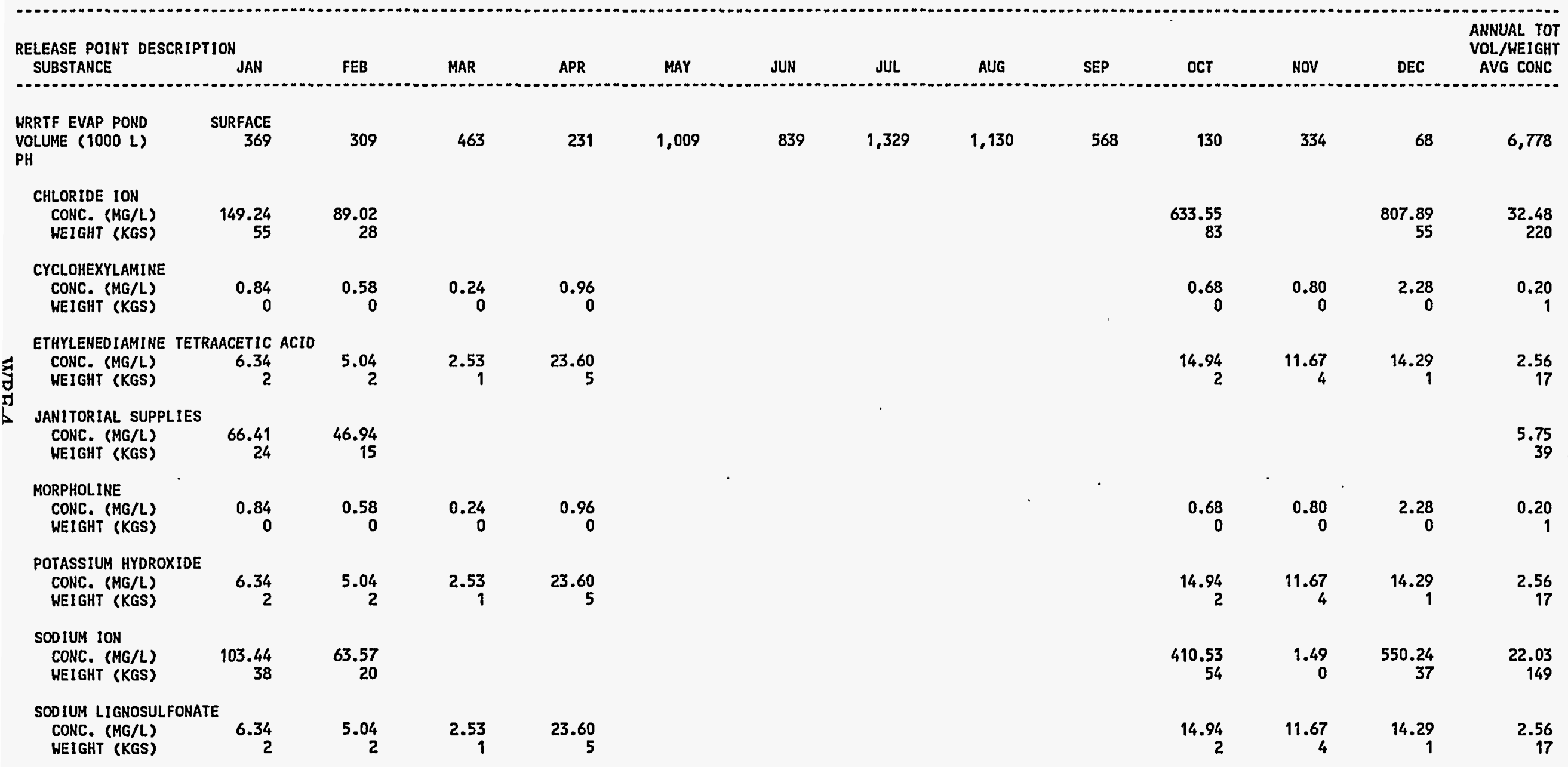


FOR JANUARY THROUGH DECEMBER 1994

WRF

(ALL. VOLLUMES $=$ NEAREST THOUSAND LITERS)

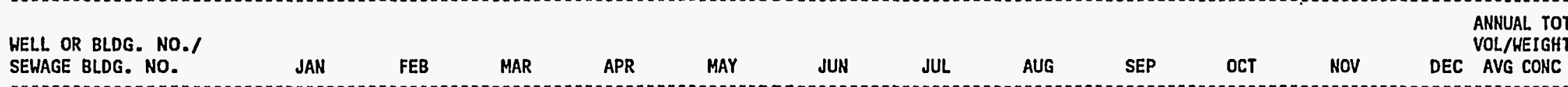

\section{WATER DATA}

WRF HATER PUMPED (L)

WATER PUMPED (L)

$\begin{array}{ccc}461 & 386 \\ 461 & 386\end{array}$

1,262

$049 \quad 1,329 \quad 1,506$

\begin{tabular}{rrrrr}
757 & 174 & 445 & 91 & 8,327 \\
\hline 757 & 174 & 445 & 91 & 8,327
\end{tabular}

WATER DISPOSED

$$
\text { AIR (L) }
$$

SURFACE (L)

SUBSURFACE (L)

578

289

1,262

\begin{tabular}{rrr}
1,049 & 1,329 & 1,506 \\
\hline 1,049 & 1,329 & 1,506
\end{tabular}

757

174

445

$91 \quad 8,327$

SEWAGE DATA

643 BUILD VOL (L)

92

1,009

$839 \quad 1,329 \quad 1,130$

568

130

334

$68 \quad 6,778$

BIOCHEMICAL OXYGEN DEMAND (BOD)

RAW CONC. (MG/L)

FINAL CONC. (MG/L)

FINAL CONC
$\%$ REMOVED

DISSOLVED OXYGEN (DO)

RAH CONC. (MG/L)

FINAL CONC. (MG/L)

INCREASED FACTOR

\section{SETTLEABLE SOLIDS (SS)}

RAH CONC. (ML/L)

FINAL CONC. (ML/L)

FINAL CONC.

\section{PH FINAL}

TOTAL SEWAGE(L)

TOTAL HATER DISPOSED(L)

116
578

289

$\begin{array}{rrrrr}252 & 210 & 332 & 376 & 189 \\ 1,262 & 1,049 & 1,661 & 1,506 & 757\end{array}$

DETAILS MAY NOT ADD UP TO TOTALS BECAUSE OF ROUNDING

0 IN A COLUMN INDICATES A TOTAL OF $<0.5$

$\begin{aligned} 0.0 & \text { IN A COLUMN INDICATES A TOTAL OF } \\ 0.00 & <0.05 \\ \text { IN A COLUMN INDICATES A TOTAL OF } & <0.005\end{aligned}$ 
PAGE: 2

SCHED. HO. INRPTOIO

IDAHO OPERATIONS OFFICE

UNITED STATES DEPARTMENT OF ENERGY

INEL. NONRADIOLOGICAL HASTE MANAGENENT INFORMATION SYSTEH

FOR JANUARY THROUGH DECEMBER 1994

WRF HATER USAGE AND DISPOSAL SUMMARY

(ALL. VOLUMES = NEAREST THOUSAND LITERS)

HRF

ANNUAL TOT
VOL/WEIGHT

HELL. OR BLDG. NO.I

SEHAGE BLDG. NO.

JAN

FEB MAR

JUN

JUL.

AUG

SEP

OCT

NoV DEC AVG CONC

ACCOUNTABILITY (\%)

100.0

90.0

100.0

100.0

100.0

100.0

125.0

100.0

100.0

100.0

100.0

100.0

103.5

DETAILS MAY NOT ADD UP TO TOTALS BECAUSE OF ROUNDING

0 IN A COLUMN INOICATES A TOTAL OF $<0.5$

0.0 IN A COLUMN INDICATES A TOTAL OF $<0.05$

0.00 IN A COLUMA INDICATES A TOTAL OF $<0.005$ 
PAGE: 1

SCHED. NO. INRPT011
IDAHO OPERATIONS OFFICE

UNITED STATES DEPARTMENT OF ENERGY

INEL NONRADIOLOGICAL. WASTE MANAGEMENT INFORMATION SYSTEM

FOR JANUARY THROUGH DECEMBER 1994

WRF FUEL OIL USAGE AND STACK EFFLUENTS SUMMARY

RPT 268-1

WRF

(ALL VALUES $=$ NEAREST THOUSAND

\begin{tabular}{|c|c|c|c|c|c|c|c|c|c|c|c|c|c|}
\hline $\begin{array}{c}\text { FUEL OIL TYPE } \\
\text { EFFLUENTS }\end{array}$ & JAN & FEB & MAR & APR & MAY & JUN & JUL & AUG & SEP & OCT & NOV & DEC & $\begin{array}{l}\text { ANNUAL } \\
\text { TOTALS }\end{array}$ \\
\hline $\begin{array}{l}\text { TYPE } 2 \\
\text { VOLUME (LITERS) } \\
\text { SO2 (KGS) } \\
\text { NOX (KGS) } \\
\text { PARTICULATES (KGS) }\end{array}$ & $\begin{array}{r}26 \\
0\end{array}$ & $\begin{array}{r}23 \\
0\end{array}$ & $\begin{array}{r}20 \\
0\end{array}$ & $\begin{array}{r}289 \\
2\end{array}$ & & & & & & $\begin{array}{l}5 \\
0\end{array}$ & $\begin{array}{r}19 \\
0\end{array}$ & $\begin{array}{r}26 \\
0\end{array}$ & $\begin{array}{r}407 \\
2\end{array}$ \\
\hline $\begin{array}{l}\text { GRAND TOTALS: } \\
\text { VOLUME (LITERS) } \\
\text { SO2 (KGS) } \\
\text { NOX (KGS) }\end{array}$ & $\begin{array}{r}26 \\
0\end{array}$ & $\begin{array}{r}23 \\
0\end{array}$ & $\begin{array}{r}20 \\
0\end{array}$ & $\begin{array}{r}289 \\
2\end{array}$ & & & & & & $\begin{array}{l}5 \\
0\end{array}$ & $\begin{array}{r}19 \\
0\end{array}$ & $\begin{array}{r}26 \\
0\end{array}$ & $\begin{array}{r}407 \\
2\end{array}$ \\
\hline
\end{tabular}

